\title{
Total Synthesis of (+)-Jatrophalactam
}

Jianhong Gao, ${ }^{\dagger, \S}$ Dongyu Sun, ${ }^{\dagger, \S}$ Kuan Yu,${ }^{\dagger}$ Hujun Xie, ${ }^{\dagger}$ and Hanfeng Ding $*, \dagger$

†Department of Chemistry, Zhejiang University, Hangzhou 310058, China

${ }^{t}$ Department of Applied Chemistry, Zhejiang Gongshang University, Hangzhou

310018, China

E-mail: hfding@zju.edu.cn

\section{Supporting Information Available}

I) Experimental Procedures and Spectroscopic Data of Compounds

II) Abbreviations

III) References

IV) ${ }^{1} \mathrm{H}$ and ${ }^{13} \mathrm{C}$ NMR Spectra of Compounds 


\section{I) Experimental Procedures and Spectroscopic Data of Compounds}

General Procedures. All reactions were carried out under an argon atmosphere with dry solvents under anhydrous conditions, unless otherwise noted. Anhydrous methylene chloride $\left(\mathrm{CH}_{2} \mathrm{Cl}_{2}\right)$ was distilled before use from calcium hydride. Anhydrous benzene, diethyl ether $\left(\mathrm{Et}_{2} \mathrm{O}\right)$, tetrahydrofuran (THF) and toluene were distilled before use from sodium-benzophenone ketyl. Acetone, acetonitrile (MeCN), 1,2dimethoxyethane (DME), ethyl acetate (EtOAc), hexanes, methanol $(\mathrm{MeOH})$, and triethylamine $\left(\mathrm{Et}_{3} \mathrm{~N}\right)$ were purchased at the highest commercial quality and used without further purification. Yields refer to chromatographically homogeneous materials. Reactions were monitored by thin-layer chromatography (TLC) carried out on $0.25 \mathrm{~mm}$ E. Merck silica gel plates (60F-254) using UV light as visualizing agent and an ethanolic solution of ammonium molybdate, anisaldehyde, and heat as developing agents. E. Merck silica gel (60, paroom temperatureicle size $0.040-0.063$ mm) was used for flash column chromatography. NMR spectra were recorded on a Bruker AV-400, Bruker AV-500 or Agilent DD2-600 instrument and calibrated using residual undeuterated solvent as an internal reference. The following abbreviations were used to explain the multiplicities: $\mathrm{s}=$ singlet, $\mathrm{d}=$ doublet, $\mathrm{t}=$ triplet, $\mathrm{q}=$ quartet, $\mathrm{br}=$ broad, $\mathrm{dd}=$ doublet of doublets, $\mathrm{ddd}=$ doublet of doublet of doublets, $\mathrm{m}=$ multiplet. Melting points (m.p.) are uncorrected, and recorded on a Buchi B-540 melting point apparatus. High-resolution mass spectra (HRMS) were recorded on Waters MALDI SYNAPT G2-Si High Definition Mass Spectrometry. 
Ester 11a: To a stirred solution of $(R)-(+)-2-m_{\text {ethyl-5-hexene-1,2-diol }}^{1}$ (9, $26.0 \mathrm{~g}, 0.2$<smiles>C=CCC[C@](C)(O)COC(=O)[C@H](C)CC=C</smiles>
mol) and (S)-(+)-2-methyl-4-pentenoic $\operatorname{acid}^{2}(\mathbf{1 0}, 22.8 \mathrm{~g}$, $0.2 \mathrm{~mol})$ in $\mathrm{CH}_{2} \mathrm{Cl}_{2}(100 \mathrm{~mL})$ at $0{ }^{\circ} \mathrm{C}$ were sequentially added EDCI (38.4 g, $0.2 \mathrm{~mol})$ and 4-DMAP (2.4 g, 20.0

mmol). The resulting mixture was warmed to room temperature and stirred for $1 \mathrm{~h}$ before it was quenched with $\mathrm{H}_{2} \mathrm{O}(100 \mathrm{~mL})$. The layers were separated, and the aqueous layer was extracted with $\mathrm{CH}_{2} \mathrm{Cl}_{2}(3 \times 40 \mathrm{~mL})$. The combined organic layers were dried $\left(\mathrm{Na}_{2} \mathrm{SO}_{4}\right)$ and concentrated in vacuo. Flash column chromatography (silica gel, hexanes:EtOAc 9:1) afforded ester 11a $(38.4 \mathrm{~g}, 85 \%)$ as a colorless oil. 11a: $R_{\mathrm{f}}=0.50$ (silica gel, hexanes:EtOAc 4:1); $[\alpha]_{\mathrm{D}}{ }^{20}=+3.6\left(\mathrm{c}=1.0, \mathrm{CHCl}_{3}\right) ;{ }^{1} \mathrm{H} \mathrm{NMR}(400 \mathrm{MHz}$, $\left.\mathrm{CDCl}_{3}\right) \delta=5.88-5.70(\mathrm{~m}, 2 \mathrm{H}), 5.09-4.95(\mathrm{~m}, 4 \mathrm{H}), 3.98(\mathrm{~s}, 2 \mathrm{H}), 2.62-2.56(\mathrm{~m}, 1 \mathrm{H})$, 2.46-2.38 (m, $1 \mathrm{H}), 2.25-2.20(\mathrm{~m}, 1 \mathrm{H}), 2.17-2.11(\mathrm{~m}, 2 \mathrm{H}), 1.65-1.55(\mathrm{~m}, 2 \mathrm{H}), 1.21$ (s, $3 \mathrm{H}), 1.19 \mathrm{ppm}(\mathrm{d}, J=6.9 \mathrm{~Hz}, 3 \mathrm{H}) ;{ }^{13} \mathrm{C} \mathrm{NMR}\left(100 \mathrm{MHz}, \mathrm{CDCl}_{3}\right) \delta=176.2,138.6$, 135.6, 117.2, 114.9, 71.8, 70.9, 39.5, 38.2, 38.0, 28.1, 24.0, 16.8 ppm; HRMS (ESI): calcd for $\mathrm{C}_{13} \mathrm{H}_{22} \mathrm{O}_{3} \mathrm{Na}^{+}[\mathrm{M}+\mathrm{Na}]^{+} 249.1467$, found 249.1472 .

Acetate 8a: To a stirred solution of ester 11a $(38.4 \mathrm{~g}, 0.17 \mathrm{~mol})$ in $\mathrm{CH}_{2} \mathrm{Cl}_{2}(100 \mathrm{~mL})$ at

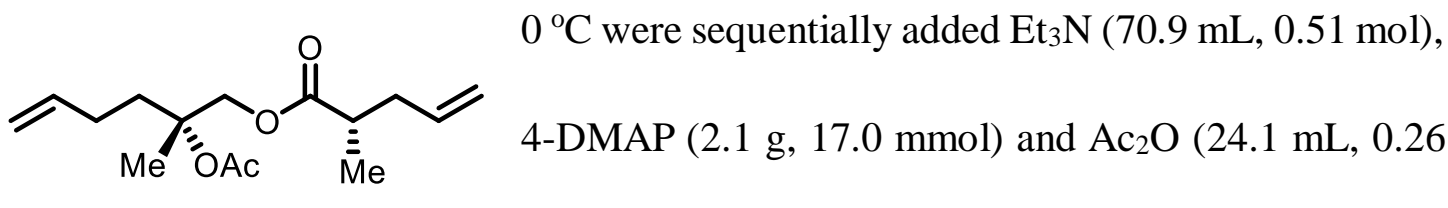
$8 a$ mol). The resulting mixture was warmed to room temperature and stirred for $6 \mathrm{~h}$ before it was quenched with $\mathrm{NaHCO}_{3}(100 \mathrm{~mL}$, sat. aq. $)$. The layers were separated, and the aqueous layer was extracted with $\mathrm{CH}_{2} \mathrm{Cl}_{2}(3 \times 40$ $\mathrm{mL})$. The combined organic layers were dried $\left(\mathrm{Na}_{2} \mathrm{SO}_{4}\right)$ and concentrated in vacuo. 
Flash column chromatography (silica gel, hexanes:EtOAc 19:1) afforded acetate 8a $(41.0 \mathrm{~g}, 90 \%)$ as a colorless oil. 8a: $R_{\mathrm{f}}=0.50\left(\right.$ silica gel, hexanes:EtOAc 9:1); $[\alpha]_{\mathrm{D}}{ }^{20}=$ $+5.0\left(\mathrm{c}=1.0, \mathrm{CHCl}_{3}\right) ;{ }^{1} \mathrm{H}$ NMR $\left(400 \mathrm{MHz}, \mathrm{CDCl}_{3}\right) \delta=5.83-5.69(\mathrm{~m}, 2 \mathrm{H}), 5.08-4.94$ (m, $4 \mathrm{H}), 4.28(\mathrm{~s}, 2 \mathrm{H}), 2.59-2.53(\mathrm{~m}, 1 \mathrm{H}), 2.44-2.37(\mathrm{~m}, 1 \mathrm{H}), 2.23-2.15(\mathrm{~m}, 1 \mathrm{H})$, 2.09-2.02 (m, $2 \mathrm{H}), 2.01-1.94(\mathrm{~m}, 1 \mathrm{H}), 1.99$ (s, $3 \mathrm{H}), 1.87-1.80$ (m, $1 \mathrm{H}), 1.45$ (s, 3 $\mathrm{H}), 1.16 \mathrm{ppm}(\mathrm{d}, J=7.0 \mathrm{~Hz}, 3 \mathrm{H}) ;{ }^{13} \mathrm{C} \mathrm{NMR}\left(100 \mathrm{MHz}, \mathrm{CDCl}_{3}\right) \delta=175.6,170.3,138.0$, 135.4, 117.1, 115.0, 82.0, 67.0, 39.4, 37.9, 35.2, 27.8, 22.2, 21.6, 16.7 ppm; HRMS (ESI): calcd for $\mathrm{C}_{15} \mathrm{H}_{24} \mathrm{O}_{4} \mathrm{Na}^{+}[\mathrm{M}+\mathrm{Na}]^{+} 291.1572$, found 291.1575 .

Lactone 7a: To a stirred solution of acetate $8 \mathbf{a}(1.0 \mathrm{~g}, 3.7 \mathrm{mmol})$ in $\mathrm{CH}_{2} \mathrm{Cl}_{2}(1.25 \mathrm{~L})$ at The resulting mixture was warmed to $40{ }^{\circ} \mathrm{C}$ and stirred for $6 \mathrm{~h}$ chromatography (silica gel, hexanes:EtOAc 19:1) afforded lactone 7a (578 mg, 65\%) as a colorless oil. 7a: $R_{\mathrm{f}}=0.40$ (silica gel, hexanes:EtOAc 9:1); $[\alpha]_{\mathrm{D}}{ }^{20}=-13.5(\mathrm{c}=1.0$, $\left.\mathrm{CHCl}_{3}\right) ;{ }^{1} \mathrm{H} \mathrm{NMR}\left(400 \mathrm{MHz}, \mathrm{CDCl}_{3}\right) \delta=5.55-5.49(\mathrm{~m}, 1 \mathrm{H}), 5.42-5.35(\mathrm{~m}, 1 \mathrm{H}), 4.35$ $(\mathrm{d}, J=11.7 \mathrm{~Hz}, 1 \mathrm{H}), 4.18(\mathrm{~d}, J=11.7 \mathrm{~Hz}, 1 \mathrm{H}), 2.62-2.55(\mathrm{~m}, 2 \mathrm{H}), 2.50-2.43(\mathrm{~m}, 2$ H), 2.07-2.02 (m, $2 \mathrm{H}), 2.05(\mathrm{~s}, 3 \mathrm{H}), 1.46(\mathrm{~s}, 3 \mathrm{H}), 1.48-1.38(\mathrm{~m}, 1 \mathrm{H}), 1.23 \mathrm{ppm}(\mathrm{d}, J$ $=7.0 \mathrm{~Hz}, 3 \mathrm{H}) ;{ }^{13} \mathrm{C} \mathrm{NMR}\left(150 \mathrm{MHz}, \mathrm{CDCl}_{3}\right) \delta=174.7,170.4,133.9,124.9,81.8,69.3$, 42.3, 38.3, 31.1, 23.0, 22.3, 21.5, 17.4 ppm; HRMS (ESI): calcd for $\mathrm{C}_{13} \mathrm{H}_{20} \mathrm{O}_{4} \mathrm{Na}^{+}[\mathrm{M}$ $+\mathrm{Na}]^{+} 263.1259$, found 263.1264 . 


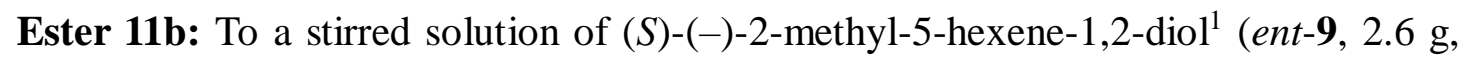

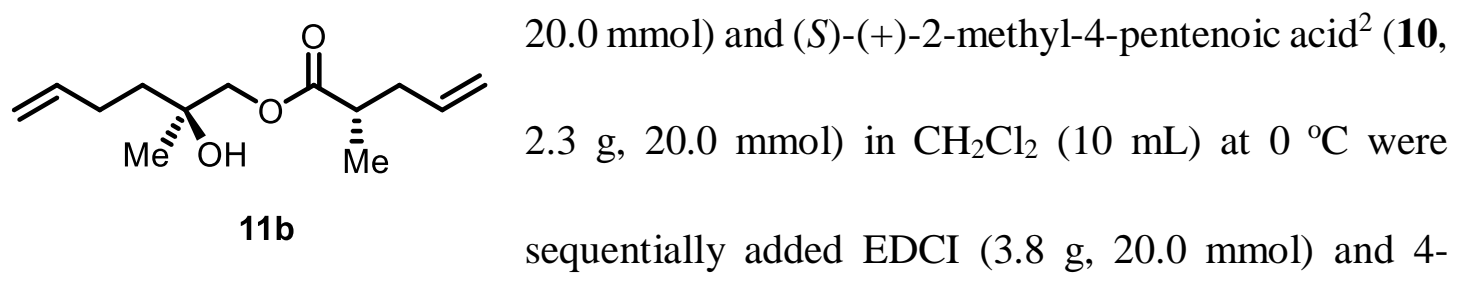

DMAP (256 mg, $2.1 \mathrm{mmol}$ ). The resulting mixture was warmed to room temperature and stirred for $1 \mathrm{~h}$ before it was quenched with $\mathrm{H}_{2} \mathrm{O}(10 \mathrm{~mL})$. The layers were separated, and the aqueous layer was extracted with $\mathrm{CH}_{2} \mathrm{Cl}_{2}(3 \times 5 \mathrm{~mL})$. The combined organic layers were dried $\left(\mathrm{Na}_{2} \mathrm{SO}_{4}\right)$ and concentrated in vacuo. Flash column chromatography (silica gel, hexanes:EtOAc 9:1) afforded ester 11b (3.6 g, 81\%) as a colorless oil. 11b: $R_{\mathrm{f}}=0.50\left(\right.$ silica gel, hexanes:EtOAc 4:1); $[\alpha]_{\mathrm{D}}{ }^{20}=+5.4\left(\mathrm{c}=1.0, \mathrm{CHCl}_{3}\right) ;{ }^{1} \mathrm{H} \mathrm{NMR}(400$ $\left.\mathrm{MHz}, \mathrm{CDCl}_{3}\right) \delta=5.83-5.66(\mathrm{~m}, 2 \mathrm{H}), 5.04-4.90(\mathrm{~m}, 4 \mathrm{H}), 3.96(\mathrm{~d}, J=11.1 \mathrm{~Hz}, 1 \mathrm{H})$, $3.91(\mathrm{~d}, J=11.1 \mathrm{~Hz}, 1 \mathrm{H}), 2.58-2.51(\mathrm{~m}, 1 \mathrm{H}), 2.41-2.34(\mathrm{~m}, 1 \mathrm{H}), 2.20-2.15(\mathrm{~m}, 2 \mathrm{H})$, 2.13-2.07 (m, $2 \mathrm{H}), 1.58-1.53(\mathrm{~m}, 2 \mathrm{H}), 1.16$ (s, $3 \mathrm{H}), 1.14$ (d, J = 6.9 Hz, $3 \mathrm{H}) \mathrm{ppm}$; ${ }^{13} \mathrm{C} \mathrm{NMR}\left(100 \mathrm{MHz}, \mathrm{CDCl}_{3}\right) \delta=176.0,138.5,135.4,117.0,114.7,71.6,70.8,39.3$, 38.0, 37.8, 27.9, 23.9, 16.7 ppm; HRMS (ESI): calcd for $\mathrm{C}_{13} \mathrm{H}_{22} \mathrm{O}_{3} \mathrm{Na}^{+}[\mathrm{M}+\mathrm{Na}]^{+}$ 249.1467, found 249.1469.

Acetate 8b: To a stirred solution of ester $\mathbf{1 1 b}(3.8 \mathrm{~g}, 17.0 \mathrm{mmol})$ in $\mathrm{CH}_{2} \mathrm{Cl}_{2}(10 \mathrm{~mL})$ at<smiles></smiles>
$0{ }^{\circ} \mathrm{C}$ were sequentially added $\mathrm{Et}_{3} \mathrm{~N}(7.1 \mathrm{~mL}, 51.0 \mathrm{mmol})$, 4-DMAP (207 mg, $1.7 \mathrm{mmol})$ and $\mathrm{Ac}_{2} \mathrm{O}(2.4 \mathrm{~mL}, 25.5$ mmol). The resulting mixture was warmed to room temperature and stirred for $6 \mathrm{~h}$ before it was quenched with $\mathrm{NaHCO}_{3}(10 \mathrm{~mL}$, sat. aq.). The layers were separated, and the aqueous layer was extracted with $\mathrm{CH}_{2} \mathrm{Cl}_{2}(3 \times 5 \mathrm{~mL})$. 
The combined organic layers were dried $\left(\mathrm{Na}_{2} \mathrm{SO}_{4}\right)$ and concentrated in vacuo. Flash column chromatography (silica gel, hexanes:EtOAc 19:1) afforded acetate $\mathbf{8 b}$ (4.2 g, 92\%) as a colorless oil. 8b: $R_{\mathrm{f}}=0.50$ (silica gel, hexanes:EtOAc 9:1); $[\alpha]_{\mathrm{D}}{ }^{20}=+6.5(\mathrm{c}$ $\left.=1.0, \mathrm{CHCl}_{3}\right) ;{ }^{1} \mathrm{H} \mathrm{NMR}\left(400 \mathrm{MHz}, \mathrm{CDCl}_{3}\right) \delta=5.77-5.62(\mathrm{~m}, 2 \mathrm{H}), 5.02-4.87(\mathrm{~m}, 4$ H), $4.22(\mathrm{~s}, 2 \mathrm{H}), 2.53-2.47(\mathrm{~m}, 1 \mathrm{H}), 2.38-2.31(\mathrm{~m}, 1 \mathrm{H}), 2.17-2.09$ (m, $1 \mathrm{H}), 2.03-$ $1.96(\mathrm{~m}, 3 \mathrm{H}), 1.92$ (s, $3 \mathrm{H}), 1.80-1.73(\mathrm{~m}, 1 \mathrm{H}), 1.38$ (s, $3 \mathrm{H}), 1.10 \mathrm{ppm}(\mathrm{d}, J=7.0 \mathrm{~Hz}$, $3 \mathrm{H}) ;{ }^{13} \mathrm{C} \mathrm{NMR}\left(100 \mathrm{MHz}, \mathrm{CDCl}_{3}\right) \delta=175.3,170.0,137.8,135.3,116.9,114.8,77.5$, 66.8, 39.3, 37.7, 35.1, 27.7, 22.1, 21.4, 16.6 ppm; HRMS (ESI): calcd for $\mathrm{C}_{15} \mathrm{H}_{24} \mathrm{O}_{4} \mathrm{Na}^{+}$ $[\mathrm{M}+\mathrm{Na}]^{+}$291.1572, found 291.1579.

Lactone 7b: To a stirred solution of acetate $8 \mathbf{b}(1.0 \mathrm{~g}, 3.7 \mathrm{mmol})$ in $\mathrm{CH}_{2} \mathrm{Cl}_{2}(1.25 \mathrm{~L})$ at

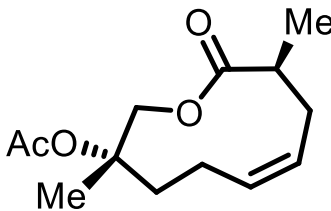

$7 \mathrm{~b}$ room temperature was added Grubbs I cat. $(310 \mathrm{mg}, 0.37 \mathrm{mmol})$. The resulting mixture was warmed to $40^{\circ} \mathrm{C}$ and stirred for $6 \mathrm{~h}$. The mixture was concentrated in vacuo. Flash column chromatography (silica gel, hexanes:EtOAc 19:1) afforded lactone 7b (489 mg, 55\%) as a colorless oil. 7b: $R_{\mathrm{f}}=0.40$ (silica gel, hexanes:EtOAc 9:1); $[\alpha]_{\mathrm{D}}{ }^{20}=+72.0(\mathrm{c}=1.0$, $\left.\mathrm{CHCl}_{3}\right) ;{ }^{1} \mathrm{H} \mathrm{NMR}\left(400 \mathrm{MHz}, \mathrm{CDCl}_{3}\right) \delta=5.58-5.51(\mathrm{~m}, 1 \mathrm{H}), 5.46-5.38(\mathrm{~m}, 4 \mathrm{H}), 4.38$ $(\mathrm{d}, J=11.7 \mathrm{~Hz}, 1 \mathrm{H}), 4.20(\mathrm{~d}, J=11.7 \mathrm{~Hz}, 1 \mathrm{H}), 2.74-2.67(\mathrm{~m}, 1 \mathrm{H}), 2.65-2.63(\mathrm{~m}, 1$ H), 2.47-2.40 (m, $2 \mathrm{H}), 2.20-2.05(\mathrm{~m}, 2 \mathrm{H}), 2.01(\mathrm{~s}, 3 \mathrm{H}), 1.50-1.45$ (m, $1 \mathrm{H}), 1.46$ (s, $3 \mathrm{H}), 1.19 \mathrm{ppm}(\mathrm{d}, J=7.0 \mathrm{~Hz}, 1 \mathrm{H}) ;{ }^{13} \mathrm{C} \mathrm{NMR}\left(150 \mathrm{MHz}, \mathrm{CDCl}_{3}\right) \delta=174.6,170.5$, 134.2, 123.8, 82.0, 69.0, 40.5, 38.1, 30.1, 23.0, 22.4, 22.0, 15.9 ppm; HRMS (ESI): calcd for $\mathrm{C}_{13} \mathrm{H}_{20} \mathrm{O}_{4} \mathrm{Na}^{+}[\mathrm{M}+\mathrm{Na}]^{+}$263.1259, found 263.1263. 
Dibromocyclopropanes 12 and 9,11-diepi-12: To a stirred solution of lactone 7a (9.6

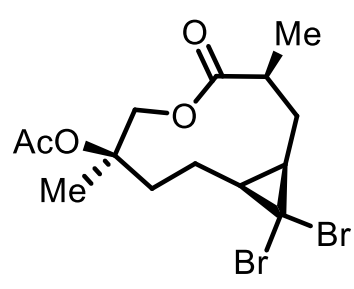

12

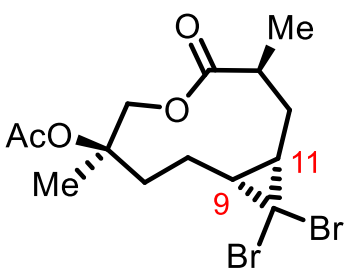

9,11-diepi-12 $\mathrm{g}, 40.0 \mathrm{mmol})$ in $\mathrm{CH}_{2} \mathrm{Cl}_{2}(50 \mathrm{~mL})$ at 0 ${ }^{\circ} \mathrm{C} \quad$ were sequentially added

$\mathrm{Et}_{3} \mathrm{~N}(\mathrm{Bn}) \mathrm{Cl}(1.8 \mathrm{~g}, 8.0 \mathrm{mmol}), \mathrm{CHBr}_{3}$ $(70.0 \mathrm{~mL}, 0.8 \mathrm{~mol})$ and $\mathrm{NaOH}(32.0$ $\mathrm{mL}, 25.0 \mathrm{M}$ aq., $0.8 \mathrm{~mol}$,). The resulting mixture was warmed to room temperature and stirred for $6 \mathrm{~h}$ before it was diluted with $\mathrm{H}_{2} \mathrm{O}(100 \mathrm{~mL})$ and $\mathrm{CH}_{2} \mathrm{Cl}_{2}(100 \mathrm{~mL})$. The layers were separated, and the aqueous layer was extracted with $\mathrm{CH}_{2} \mathrm{Cl}_{2}(3 \times 40 \mathrm{~mL})$. The combined organic layers were dried $\left(\mathrm{Na}_{2} \mathrm{SO}_{4}\right)$ and concentrated in vacuo. Flash column chromatography (silica gel, hexanes:EtOAc 19:1) afforded dibromocyclopropane $12(11.9 \mathrm{~g}$, 73\%) as a white solid, along with dibromocyclopropane 9,11-diepi-12 $(2.0 \mathrm{~g}, 13 \%)$ as a colorless oil. 12: $R_{\mathrm{f}}=0.38$ (silica gel, hexanes:EtOAc 9:1); $[\alpha]_{\mathrm{D}}{ }^{20}=-16.0\left(\mathrm{c}=1.0, \mathrm{CHCl}_{3}\right) ;$ m.p. $=102-104{ }^{\circ} \mathrm{C}$ (EtOAc/hexanes); ${ }^{1} \mathrm{H}$ NMR $\left(400 \mathrm{MHz}, \mathrm{CDCl}_{3}\right) \delta=4.56(\mathrm{~d}, J=12.0 \mathrm{~Hz}, 1 \mathrm{H}), 4.05(\mathrm{~d}$, $J=12.0 \mathrm{~Hz}, 1 \mathrm{H}), 2.71-2.58(\mathrm{~m}, 2 \mathrm{H}), 2.04(\mathrm{~s}, 3 \mathrm{H}), 1.99-1.94(\mathrm{~m}, 1 \mathrm{H}), 1.79-1.74(\mathrm{~m}$, $1 \mathrm{H}), 1.73-1.69(\mathrm{~m}, 1 \mathrm{H}), 1.62-1.54(\mathrm{~m}, 2 \mathrm{H}), 1.50-1.43(\mathrm{~m}, 1 \mathrm{H}), 1.46(\mathrm{~s}, 3 \mathrm{H}), 1.40$ $1.31(\mathrm{~m}, 1 \mathrm{H}), 1.27 \mathrm{ppm}(\mathrm{d}, J=7.0 \mathrm{~Hz}, 3 \mathrm{H}),{ }^{13} \mathrm{C} \mathrm{NMR}\left(100 \mathrm{MHz}, \mathrm{CDCl}_{3}\right) \delta=174.7$, 170.5, 81.8, 70.3, 41.0, 36.1, 35.1, 35.0, 33.0, 31.2, 22.8, 22.2, 20.1, 18.5 ppm; HRMS (ESI): calcd for $\mathrm{C}_{14} \mathrm{H}_{20} \mathrm{O}_{4} \mathrm{NaBr}_{2}{ }^{+}[\mathrm{M}+\mathrm{Na}]^{+}$432.9626, found 432.9630 .

9,11-diepi-12: $R_{\mathrm{f}}=0.35$ (silica gel, hexanes:EtOAc 9:1); $[\alpha]_{\mathrm{D}}{ }^{20}=+32.2\left(\mathrm{c}=1.0, \mathrm{CHCl}_{3}\right)$; ${ }^{1} \mathrm{H} \mathrm{NMR}\left(400 \mathrm{MHz}, \mathrm{CDCl}_{3}\right) \delta=5.08(\mathrm{~d}, J=11.4 \mathrm{~Hz}, 1 \mathrm{H}), 3.80(\mathrm{~d}, J=11.4 \mathrm{~Hz}, 1 \mathrm{H})$, 2.83-2.80 (m, $1 \mathrm{H}), 2.14-2.10(\mathrm{~m}, 1 \mathrm{H}), 2.03-2.00(\mathrm{~m}, 1 \mathrm{H}), 1.96(\mathrm{~s}, 3 \mathrm{H}), 1.86-1.66$ 
$(\mathrm{m}, 5 \mathrm{H}), 1.55(\mathrm{~s}, 3 \mathrm{H}), 1.47-1.38(\mathrm{~m}, 1 \mathrm{H}), 1.30 \mathrm{ppm}(\mathrm{d}, J=7.2 \mathrm{~Hz}, 3 \mathrm{H}) ;{ }^{13} \mathrm{C} \mathrm{NMR}$ $\left(100 \mathrm{MHz}, \mathrm{CDCl}_{3}\right) \delta=173.8,170.0,82.3,68.1,37.9,37.1,36.6,34.4,29.4,29.1,22.7$, 22.4 (2C), 15.0 ppm; HRMS (ESI): calcd for $\mathrm{C}_{14} \mathrm{H}_{20} \mathrm{O}_{4} \mathrm{NaBr}_{2}{ }^{+}[\mathrm{M}+\mathrm{Na}]^{+}$432.9626, found 432.9634 .

Alternatively, dibromocyclopropanes 12 and 9,11-diepi-12 could also be obtained according to the following procedure:

To a stirred solution of lactone $7 \mathbf{a}(720 \mathrm{mg}, 3.0 \mathrm{mmol})$ in toluene $(15 \mathrm{~mL})$ at room temperature was added $\mathrm{PhHgCBr}_{3}(3.25 \mathrm{~g}, 6.0 \mathrm{mmol})$. The resulting mixture was warmed to $80{ }^{\circ} \mathrm{C}$ and stirred for $12 \mathrm{~h}$ before it was cooled to room temperature and concentrated in vacuo. Flash column chromatography (silica gel, hexanes:EtOAc 19:1) afforded 12 (344 mg, 28\%) and 9,11-diepi-12 (62 mg, 5\%).

Dibromocyclopropanes 6-epi-12 and 6,9,11-triepi-12: To a stirred solution of lactone

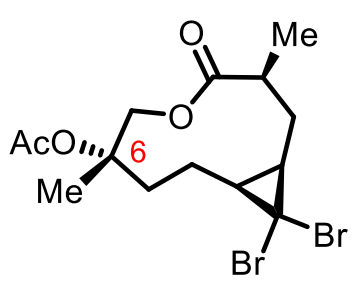

6-epi-12

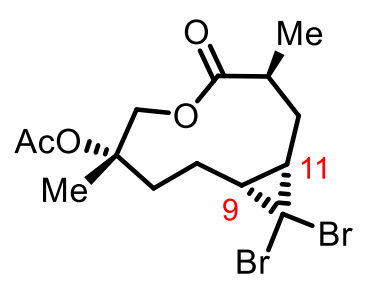

6,9,11-triepi-12
$7 \mathbf{b}(1.0 \mathrm{~g}, 4.1 \mathrm{mmol})$ in $\mathrm{CH}_{2} \mathrm{Cl}_{2}(5 \mathrm{~mL})$ at $\quad 0 \quad{ }^{\circ} \mathrm{C}$ were sequentially added $\mathrm{Et}_{3} \mathrm{~N}(\mathrm{Bn}) \mathrm{Cl} \quad(182 \mathrm{mg}, \quad 0.8 \mathrm{mmol})$, $\mathrm{CHBr}_{3}(7.0 \mathrm{~mL}, 80.0 \mathrm{mmol})$ and $\mathrm{NaOH}$

(3.2 $\mathrm{mL}, 25 \mathrm{M}$ aq., $80.0 \mathrm{mmol}$ ). The resulting mixture was warmed to room temperature and stirred for $6 \mathrm{~h}$ before it was diluted with $\mathrm{CH}_{2} \mathrm{Cl}_{2}(10 \mathrm{~mL})$ and $\mathrm{H}_{2} \mathrm{O}(10 \mathrm{~mL})$. The layers were separated, and the aqueous layer was extracted with $\mathrm{CH}_{2} \mathrm{Cl}_{2}(3 \times 5 \mathrm{~mL})$. The combined organic layers were dried $\left(\mathrm{Na}_{2} \mathrm{SO}_{4}\right)$ and concentrated in vacuo. Flash column chromatography (silica gel, hexanes:EtOAc 19:1) afforded dibromocyclopropane 6-epi-12 (602 $\mathrm{mg}, 37 \%)$ as a white solid, along with 
dibromocyclopropane 6,9,11-triepi-12 $(736 \mathrm{mg}, 45 \%)$ as a colorless oil. 6-epi-12: $R_{\mathrm{f}}=$ 0.35 (silica gel, hexanes:EtOAc 9:1); m.p. $=101-103{ }^{\circ} \mathrm{C}($ EtOAc/hexanes $) ;[\alpha]_{\mathrm{D}}{ }^{20}=-$ $42.6\left(\mathrm{c}=1.0, \mathrm{CHCl}_{3}\right) ;{ }^{1} \mathrm{H} \mathrm{NMR}\left(400 \mathrm{MHz}, \mathrm{CDCl}_{3}\right) \delta=4.93(\mathrm{~d}, J=11.5 \mathrm{~Hz}, 1 \mathrm{H}), 3.98$ $(\mathrm{d}, J=11.5 \mathrm{~Hz}, 1 \mathrm{H}), 2.67-2.59(\mathrm{~m}, 1 \mathrm{H}), 2.25-2.17(\mathrm{~m}, 1 \mathrm{H}), 1.97(\mathrm{~s}, 3 \mathrm{H}), 1.92-1.87$ (m, $3 \mathrm{H}), 1.82-1.76(\mathrm{~m}, 1 \mathrm{H}), 1.72-1.67$ (m, $1 \mathrm{H}), 1.53(\mathrm{~s}, 3 \mathrm{H}), 1.41-1.31(\mathrm{~m}, 2 \mathrm{H})$, $1.24 \mathrm{ppm}(\mathrm{d}, J=7.0 \mathrm{~Hz}, 3 \mathrm{H}) ;{ }^{13} \mathrm{C} \mathrm{NMR}\left(100 \mathrm{MHz}, \mathrm{CDCl}_{3}\right) \delta=174.3,170.1,82.2$, 67.4, 40.0, 38.4, 36.8, 34.0, 33.7, 31.4, 23.0, 22.8, 22.4, 18.4 ppm; HRMS (ESI): calcd for $\mathrm{C}_{14} \mathrm{H}_{20} \mathrm{O}_{4} \mathrm{NaBr}_{2}{ }^{+}[\mathrm{M}+\mathrm{Na}]^{+} 432.9626$, found 432.9635 .

6,9,11-triepi-12: $R_{\mathrm{f}}=0.38$ (silica gel, hexanes:EtOAc 9:1); $[\alpha]_{\mathrm{D}}{ }^{20}=+15.5(\mathrm{c}=1.0$, $\left.\mathrm{CHCl}_{3}\right) ;{ }^{1} \mathrm{H} \mathrm{NMR}\left(400 \mathrm{MHz}, \mathrm{CDCl}_{3}\right) \delta=4.67(\mathrm{~d}, J=12.1 \mathrm{~Hz}, 1 \mathrm{H}), 3.96(\mathrm{~d}, J=12.1$ Hz, $1 \mathrm{H}), 2.86-2.83$ (m, $1 \mathrm{H}), 2.66-2.58$ (m, $1 \mathrm{H}), 2.04$ (s, $3 \mathrm{H}), 1.92-1.87$ (m, $2 \mathrm{H})$, 1.78-1.78 (m, $2 \mathrm{H}), 1.57-1.52$ (m, $1 \mathrm{H}), 1.46$ (s, $3 \mathrm{H}), 1.48-1.43$ (m, $1 \mathrm{H}), 1.41-1.33$ $(\mathrm{m}, 1 \mathrm{H}), 1.28 \mathrm{ppm}(\mathrm{d}, J=7.0 \mathrm{~Hz}, 3 \mathrm{H}) ;{ }^{13} \mathrm{C} \mathrm{NMR}\left(100 \mathrm{MHz}, \mathrm{CDCl}_{3}\right) \delta=174.7,170.6$, 81.9, 70.4, 36.3, 35.9, 35.7, 34.9, 29.8, 28.8, 22.7, 22.2, 19.9, 14.8 ppm; HRMS (ESI): calcd for $\mathrm{C}_{14} \mathrm{H}_{20} \mathrm{O}_{4} \mathrm{NaBr}_{2}{ }^{+}[\mathrm{M}+\mathrm{Na}]^{+}$432.9626, found 432.9634 .

Dimethylcyclopropane 13: To a stirred solution of CuSCN (36.5 g, $0.3 \mathrm{~mol})$ in $\mathrm{Et}_{2} \mathrm{O}$

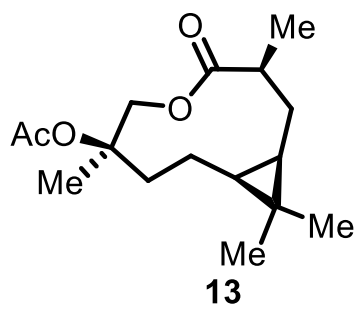
$(100 \mathrm{~mL})$ at $-20^{\circ} \mathrm{C}$ was slowly added $\mathrm{MeLi}(312 \mathrm{~mL}, 1.6 \mathrm{M}$ in $\left.\mathrm{Et}_{2} \mathrm{O}, 0.5 \mathrm{~mol}\right)$. The resulting mixture was stirred at $-20{ }^{\circ} \mathrm{C}$ for 30 min before a solution of dibromocyclopropane 12 (10.3 g, $25.0 \mathrm{mmol})$ in $\mathrm{HMPA}(13.1 \mathrm{~mL}, 75.0 \mathrm{mmol})$ and $\mathrm{Et}_{2} \mathrm{O}(20 \mathrm{~mL})$ was added. The mixture was stirred at $-20{ }^{\circ} \mathrm{C}$ for further $6 \mathrm{~h}$ before it was cooled to $50{ }^{\circ} \mathrm{C}$ and added $\mathrm{MeI}(31.3 \mathrm{~mL}, 0.5 \mathrm{~mol})$. The resulting mixture was stirred at $-50{ }^{\circ} \mathrm{C}$ 
for an additional $1 \mathrm{~h}$ before it was quenched with $\mathrm{NH}_{4} \mathrm{Cl}(100 \mathrm{~mL}$, sat. aq.). The layers were separated, and the aqueous layer was extracted with $\mathrm{Et}_{2} \mathrm{O}(3 \times 40 \mathrm{~mL})$. The combined organic layers were dried $\left(\mathrm{Na}_{2} \mathrm{SO}_{4}\right)$ and concentrated in vacuo. Flash column chromatography (silica gel, hexanes:EtOAc 19:1) afforded dimethylcyclopropane 13 $(4.7 \mathrm{~g}, 67 \%)$ as a colorless oil. 13: $R_{\mathrm{f}}=0.60$ (silica gel, hexanes:EtOAc 9:1); $[\alpha]_{\mathrm{D}}{ }^{20}=-$ $12.0\left(\mathrm{c}=1.0, \mathrm{CHCl}_{3}\right) ;{ }^{1} \mathrm{H} \mathrm{NMR}\left(400 \mathrm{MHz}, \mathrm{CDCl}_{3}\right) \delta=4.69(\mathrm{~d}, J=12.0 \mathrm{~Hz}, 1 \mathrm{H}), 3.93$ $(\mathrm{d}, J=12.0 \mathrm{~Hz}, 1 \mathrm{H}), 2.60-2.50(\mathrm{~m}, 2 \mathrm{H}), 2.04(\mathrm{~s}, 3 \mathrm{H}), 1.70-1.65(\mathrm{~m}, 1 \mathrm{H}), 1.50-1.34$ (m, $3 \mathrm{H}), 1.45(\mathrm{~s}, 3 \mathrm{H}), 1.27-1.16(\mathrm{~m}, 1 \mathrm{H}), 1.22(\mathrm{~d}, J=7.0 \mathrm{~Hz}, 3 \mathrm{H}), 0.96$ (s, $3 \mathrm{H}), 0.79$ (s, $3 \mathrm{H}), 0.46-0.41(\mathrm{~m}, 1 \mathrm{H}), 0.36-0.31 \mathrm{ppm}(\mathrm{m}, 1 \mathrm{H}) ;{ }^{13} \mathrm{C} \mathrm{NMR}\left(100 \mathrm{MHz}, \mathrm{CDCl}_{3}\right) \delta$ $=175.9,170.7,82.6,70.3,42.7,37.8,28.9(2 \mathrm{C}), 28.2,25.6,22.8,22.2,18.6,16.9,16.6$, 15.1 ppm; HRMS (ESI): calcd for $\mathrm{C}_{16} \mathrm{H}_{26} \mathrm{O}_{4} \mathrm{Na}^{+}[\mathrm{M}+\mathrm{Na}]^{+}$305.1729, found 305.1732. Ester 14: To a stirred solution of dimethylcyclopropane 13 (4.6 g, $16.0 \mathrm{mmol})$ in THF

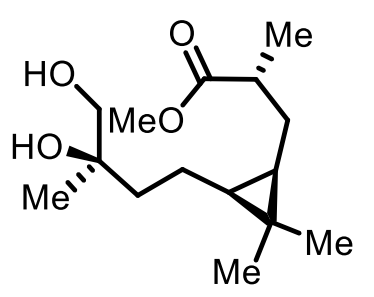

14 $(100 \mathrm{~mL})$ at $-78{ }^{\circ} \mathrm{C}$ was slowly added LDA (100 $\mathrm{mL}, 0.8 \mathrm{M}$ in THF, $80.0 \mathrm{mmol}$ ). The resulting mixture was stirred at $-78{ }^{\circ} \mathrm{C}$ for $4 \mathrm{~h}$ before it was quenched with $\mathrm{H}_{2} \mathrm{O}(50 \mathrm{~mL})$ and $\mathrm{MeOH}$ $(50 \mathrm{~mL})$. The mixture was warmed to $50{ }^{\circ} \mathrm{C}$ and stirred for further $6 \mathrm{~h}$ before it was acidified with $\mathrm{HCl}(85 \mathrm{~mL}, 1.0 \mathrm{M}$, aq., $85 \mathrm{mmol})$. The layers were separated, and the aqueous layer was extracted with $\mathrm{Et}_{2} \mathrm{O}(3 \times 40 \mathrm{~mL})$. The combined organic layers were dried $\left(\mathrm{Na}_{2} \mathrm{SO}_{4}\right)$ and concentrated in vacuo. Flash column chromatography (silica gel, hexanes:EtOAc 1:1) afforded ester 14 (3.5 g, 80\%) as a colorless oil. 14: $R_{\mathrm{f}}=0.40$ (silica gel, hexanes:EtOAc $\left.1: 1\right) ;[\alpha]_{\mathrm{D}}{ }^{20}=-60.0(\mathrm{c}=1.0$, $\left.\mathrm{CHCl}_{3}\right) ;{ }^{1} \mathrm{H} \mathrm{NMR}\left(400 \mathrm{MHz}, \mathrm{CDCl}_{3}\right) \delta=3.68(\mathrm{~s}, 3 \mathrm{H}), 3.47(\mathrm{~d}, J=10.8 \mathrm{~Hz}, 1 \mathrm{H}), 3.41$ 
(d, $J=10.8 \mathrm{~Hz}, 1 \mathrm{H}), 2.51-2.42(\mathrm{~m}, 1 \mathrm{H}), 1.95$ (brs, $1 \mathrm{H}), 1.83$ (brs, $1 \mathrm{H}), 1.61-1.51$ (m, $3 \mathrm{H}), 1.44-1.37(\mathrm{~m}, 1 \mathrm{H}), 1.35-1.28(\mathrm{~m}, 1 \mathrm{H}), 1.27-1.21(\mathrm{~m}, 1 \mathrm{H}), 1.17(\mathrm{~d}, J=7.0$ $\mathrm{Hz}, 3 \mathrm{H}), 1.16$ (s, $3 \mathrm{H}), 1.01$ (s, $3 \mathrm{H}), 0.90$ (s, $3 \mathrm{H}), 0.47-0.39 \mathrm{ppm}(\mathrm{m}, 2 \mathrm{H}) ;{ }^{13} \mathrm{C} \mathrm{NMR}$ $\left(100 \mathrm{MHz} \mathrm{CDCl}_{3}\right) \delta=177.5,73.0,69.8,51.7,40.2,39.2,29.4,28.8,27.0,24.3,23.3$, 18.9, 17.2, 17.1, 14.8 ppm; HRMS (ESI): calcd for $\mathrm{C}_{15} \mathrm{H}_{28} \mathrm{O}_{4} \mathrm{Na}^{+}[\mathrm{M}+\mathrm{Na}]^{+}$295.1885, found 295.1886 .

Diol 15: To a stirred solution of ester 14 (3.5 g, $12.9 \mathrm{mmol})$ in $\mathrm{CH}_{2} \mathrm{Cl}_{2}(20 \mathrm{~mL})$ at room<smiles>COC(=O)[C@H](C)CC1C(CCC(C)(O)O)C1(C)C</smiles>

15 temperature were sequentially added $\mathrm{Et}_{3} \mathrm{~N}(5.4 \mathrm{~mL}, 38.7 \mathrm{mmol})$, 4-DMAP (305 mg, $2.5 \mathrm{mmol})$ and $\mathrm{TsCl}(3.7 \mathrm{~g}, 19.4 \mathrm{mmol})$. The resulting mixture was stirred for $6 \mathrm{~h}$ before it was quenched with $\mathrm{NaHCO}_{3}(100 \mathrm{~mL}$, sat. aq.). The layers were separated, and the aqueous layer was extracted with $\mathrm{CH}_{2} \mathrm{Cl}_{2}(3 \times 10 \mathrm{~mL})$. The combined organic layers were dried $\left(\mathrm{Na}_{2} \mathrm{SO}_{4}\right)$ and concentrated in vacuo to afford the crude tosylate as a pale yellow oil. To a stirred solution of this crude in $\mathrm{MeOH}(20 \mathrm{~mL})$ at room temperature was added $\mathrm{K}_{2} \mathrm{CO}_{3}(8.9 \mathrm{~g}, 64.5 \mathrm{mmol})$. The resulting mixture was stirred for $30 \mathrm{~min}$ before it was diluted with $\mathrm{H}_{2} \mathrm{O}(20 \mathrm{~mL})$ and $\mathrm{CH}_{2} \mathrm{Cl}_{2}(20 \mathrm{~mL})$. The layers were separated, and the aqueous layer was extracted with $\mathrm{CH}_{2} \mathrm{Cl}_{2}(3 \times 10 \mathrm{~mL})$. The combined organic layers were dried $\left(\mathrm{Na}_{2} \mathrm{SO}_{4}\right)$ and concentrated in vacuo to afford the crude epoxide as a pale yellow oil.

To a stirred solution of the epoxide (crude, obtained above) in DME (20 mL) at room temperature was added $\mathrm{H}_{2} \mathrm{SO}_{4}(20 \mathrm{~mL}, 0.1 \mathrm{M}$ aq., $2.0 \mathrm{mmol})$. The resulting mixture was stirred for 30 min before it was diluted with $\mathrm{H}_{2} \mathrm{O}(20 \mathrm{~mL})$ and $\mathrm{CH}_{2} \mathrm{Cl}_{2}(20$ 
$\mathrm{mL})$. The layers were separated, and the aqueous layer was extracted with $\mathrm{CH}_{2} \mathrm{Cl}_{2}(3 \times$ $10 \mathrm{~mL})$. The combined organic layers were dried $\left(\mathrm{Na}_{2} \mathrm{SO}_{4}\right)$ and concentrated in vacuo. Flash column chromatography (silica gel, hexanes:EtOAc 1:1) afforded diol 15 (2.6 g, $73 \%$ over two steps) as a colorless oil. 15: $R_{\mathrm{f}}=0.40$ (silica gel, hexanes:EtOAc 1:1); $[\alpha]_{\mathrm{D}}^{20}=-20.0\left(\mathrm{c}=1.0, \mathrm{CHCl}_{3}\right) ;{ }^{1} \mathrm{H} \mathrm{NMR}\left(400 \mathrm{MHz}, \mathrm{CDCl}_{3}\right) \delta=3.68(\mathrm{~s}, 3 \mathrm{H}), 3.47(\mathrm{~d}$, $J=10.8 \mathrm{~Hz}, 1 \mathrm{H}), 3.41(\mathrm{~d}, J=10.8 \mathrm{~Hz}, 1 \mathrm{H}), 2.51-2.42(\mathrm{~m}, 1 \mathrm{H}), 1.95$ (brs, $1 \mathrm{H}), 1.83$ (brs, $1 \mathrm{H}), 1.61-1.51(\mathrm{~m}, 3 \mathrm{H}), 1.44-1.37$ (m, $1 \mathrm{H}), 1.35-1.28(\mathrm{~m}, 1 \mathrm{H}), 1.27-1.21(\mathrm{~m}$, $1 \mathrm{H}), 1.17(\mathrm{~d}, J=7.0 \mathrm{~Hz}, 3 \mathrm{H}), 1.16(\mathrm{~s}, 3 \mathrm{H}), 1.01$ (s, $3 \mathrm{H}), 0.90(\mathrm{~s}, 3 \mathrm{H}), 0.47-0.39 \mathrm{ppm}$ $(\mathrm{m}, 2 \mathrm{H}) ;{ }^{13} \mathrm{C} \mathrm{NMR}\left(100 \mathrm{MHz}, \mathrm{CDCl}_{3}\right) \delta=177.5,73.0,69.8,51.7,40.2,39.2,29.4$, 28.8, 27.0, 24.3, 23.3, 18.9, 17.2, 17.1, 14.8 ppm; HRMS (ESI): calcd for $\mathrm{C}_{15} \mathrm{H}_{28} \mathrm{O}_{4} \mathrm{Na}^{+}$ $[\mathrm{M}+\mathrm{Na}]^{+} 295.1885$, found 295.1888.

Aldehyde 16: To a stirred solution of diol 15 (2.5 g, $9.2 \mathrm{mmol})$ in $\mathrm{CH}_{2} \mathrm{Cl}_{2}(40 \mathrm{~mL})$ and

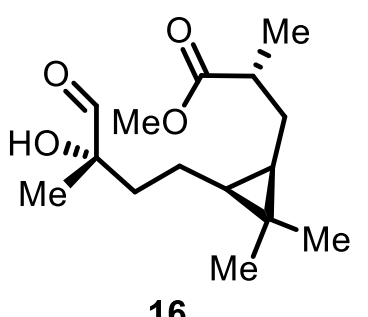

16

$\mathrm{H}_{2} \mathrm{O}(40 \mathrm{~mL})$ at room temperature were sequentially added TEMPO (296 mg, 1.9 mmol), $\mathrm{KBr}(5.5 \mathrm{~g}, 46.0 \mathrm{mmol}), \mathrm{NaHCO}_{3}$ (3.9 g, $46.0 \mathrm{mmol})$ and $\mathrm{NaClO}(10.0 \mathrm{~mL}, 1.0 \mathrm{M}$ aq., $10.0 \mathrm{mmol})$. The resulting mixture was stirred for $30 \mathrm{~min}$. The layers were separated, and the aqueous layer was extracted with $\mathrm{CH}_{2} \mathrm{Cl}_{2}(3 \times 20 \mathrm{~mL})$. The combined organic layers were dried $\left(\mathrm{Na}_{2} \mathrm{SO}_{4}\right)$ and concentrated in vacuo. Flash column chromatography (silica gel, hexanes:EtOAc 9:1) afforded aldehyde $\mathbf{1 6}(2.2 \mathrm{~g}, 88 \%)$ as a colorless oil. 16: $R_{\mathrm{f}}=0.40$ ( silica gel, hexanes:EtOAc 4:1); $[\alpha]_{\mathrm{D}}{ }^{20}=-12.0(\mathrm{c}=1.0$, $\left.\mathrm{CHCl}_{3}\right) ;{ }^{1} \mathrm{H} \mathrm{NMR}\left(400 \mathrm{MHz}, \mathrm{CDCl}_{3}\right) \delta=9.51(\mathrm{~s}, 1 \mathrm{H}), 3.68(\mathrm{~s}, 3 \mathrm{H}), 3.10(\mathrm{~s}, 1 \mathrm{H})$, 2.47-1.42 (m, $1 \mathrm{H}), 1.73-1.56$ (m, $3 \mathrm{H}), 1.39-1.33$ (m, $2 \mathrm{H}), 1.34-1.28$ (m, $1 \mathrm{H}), 1.30$ 
(s, $3 \mathrm{H}), 1.16(\mathrm{~d}, J=7.0 \mathrm{~Hz}, 3 \mathrm{H}), 1.00$ (s, $3 \mathrm{H}), 0.86$ (s, $3 \mathrm{H}), 0.43-0.39 \mathrm{ppm}(\mathrm{m}, 2 \mathrm{H})$;

${ }^{13} \mathrm{C} \mathrm{NMR}\left(100 \mathrm{MHz}, \mathrm{CDCl}_{3}\right) \delta=203.9,177.3,78.0,51.7,40.2,37.7,29.3,28.8,26.7$, 24.4, 22.8, 18.4, 17.4, 17.1, 14.8 ppm; HRMS (ESI): calcd for $\mathrm{C}_{15} \mathrm{H}_{26} \mathrm{O}_{4} \mathrm{Na}^{+}[\mathrm{M}+\mathrm{Na}]^{+}$ 293.1729, found 293.1732.

Acid 4: To a stirred solution of $\mathrm{Ph}_{3} \mathrm{PCH}_{3} \mathrm{Br}(4.3 \mathrm{~g}, 12.0 \mathrm{mmol})$ in $\mathrm{THF}(20 \mathrm{~mL})$ at $0{ }^{\circ} \mathrm{C}$<smiles>C=C[C@](C)(O)CCC1C(C[C@H](C)C(=O)O)C1(C)C</smiles>

4 was added $t$-BuOK $(10.0 \mathrm{~mL}, 1.0 \mathrm{M}$ in THF, $10.0 \mathrm{mmol})$. The resulting mixture was stirred at $0{ }^{\circ} \mathrm{C}$ for 30 min before it was slowly added to a solution of aldehyde $\mathbf{1 6}(1.1 \mathrm{~g}, 4.0 \mathrm{mmol})$ in THF $(10 \mathrm{~mL})$. The reaction was stirred at $0{ }^{\circ} \mathrm{C}$ for further $5 \mathrm{~h}$ before it were added $\mathrm{MeOH}(20 \mathrm{~mL})$ and $\mathrm{LiOH}(10.0 \mathrm{~mL}, 4.0 \mathrm{M}$ aq., $40.0 \mathrm{mmol})$. The resulting mixture was stirred at room temperature for an additional $6 \mathrm{~h}$ before it was acidified with $\mathrm{HCl}(50 \mathrm{~mL}, 1.0 \mathrm{M}$ aq., $50.0 \mathrm{mmol})$. The layers were separated, and the aqueous layer was extracted with $\mathrm{Et}_{2} \mathrm{O}(3 \times 20 \mathrm{~mL})$. The combined organic layers were dried $\left(\mathrm{Na}_{2} \mathrm{SO}_{4}\right)$ and concentrated in vacuo. Flash column chromatography (silica gel, hexanes:EtOAc 9:1) afforded acid $4(813 \mathrm{mg}, 80 \%)$ as a colorless oil. $4: R_{\mathrm{f}}=0.55($ silica gel, hexanes:EtOAc 4:1); $[\alpha]_{\mathrm{D}}{ }^{20}=-0.5\left(\mathrm{c}=1.0, \mathrm{CHCl}_{3}\right) ;{ }^{1} \mathrm{H} \mathrm{NMR}\left(400 \mathrm{MHz}, \mathrm{CDCl}_{3}\right)$ $\delta=5.90(\mathrm{dd}, J=17.4,10.8 \mathrm{~Hz}, 1 \mathrm{H}), 5.20(\mathrm{dd}, J=17.4,1.2 \mathrm{~Hz}, 1 \mathrm{H}), 5.05(\mathrm{dd}, J=$ 10.8, 1.3 Hz, $1 \mathrm{H}), 2.51-2.46$ (m, $1 \mathrm{H}), 1.64-1.52$ (m, $3 \mathrm{H}), 1.50-1.41$ (m, $1 \mathrm{H}), 1.35-$ $1.25(\mathrm{~m}, 1 \mathrm{H}), 1.28(\mathrm{~s}, 3 \mathrm{H}), 1.22-1.16(\mathrm{~m}, 1 \mathrm{H}), 1.20(\mathrm{~d}, J=7.0 \mathrm{~Hz}, 3 \mathrm{H}), 1.01(\mathrm{~s}, 3 \mathrm{H})$, 0.90 (s, $3 \mathrm{H}), 0.49-0.41 \mathrm{ppm}(\mathrm{m}, 2 \mathrm{H}) ;{ }^{13} \mathrm{C} \mathrm{NMR}\left(100 \mathrm{MHz}, \mathrm{CDCl}_{3}\right) \delta=182.5,145.1$, 111.9, 73.5, 42.9, 40.1, 29.4, 28.8, 27.9, 27.0, 24.2, 19.1, 17.3, 16.8, 14.9 ppm; HRMS (ESI): calcd for $\mathrm{C}_{15} \mathrm{H}_{26} \mathrm{O}_{3} \mathrm{Na}^{+}[\mathrm{M}+\mathrm{Na}]^{+} 277.1780$, found 277.1780. 
Amine 5: To a stirred solution of $\mathrm{Ph}_{3} \mathrm{PCH}_{3} \mathrm{Br}(7.2 \mathrm{~g}, 20.0 \mathrm{mmol})$ in $\mathrm{THF}(50 \mathrm{~mL})$ at 0

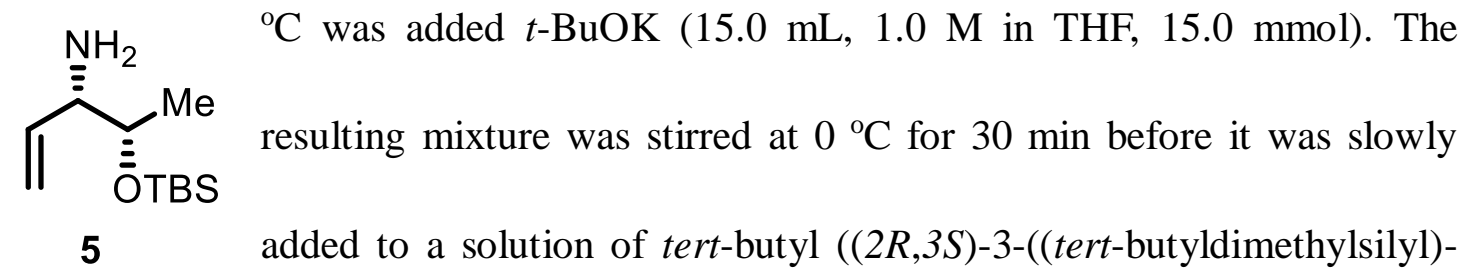
oxy)-1-oxobutan-2-yl)carbamate ${ }^{3}(3.2 \mathrm{~g}, 10.0 \mathrm{mmol})$ in THF $(50 \mathrm{~mL})$. The resulting mixture was warmed to room temperature and stirred for $5 \mathrm{~h}$ before it was quenched with $\mathrm{NH}_{4} \mathrm{Cl}$ (50 mL, sat. aq.). The layers were separated, and the aqueous layer was extracted with $\mathrm{Et}_{2} \mathrm{O}(3 \times 20 \mathrm{~mL})$. The combined organic layers were dried $\left(\mathrm{Na}_{2} \mathrm{SO}_{4}\right)$ and concentrated in vacuo to afford the crude alkene as a pale yellow oil.

To a stirred solution of the alkene (crude, obtained above) in $\mathrm{CH}_{2} \mathrm{Cl}_{2}(50 \mathrm{~mL})$ at room temperature was added TFA $(5 \mathrm{~mL})$. The mixture was stirred for $1 \mathrm{~h}$ before it was quenched with $\mathrm{NaHCO}_{3}(50 \mathrm{~mL}$, sat. aq.). The layers were separated, and the aqueous layer was extracted with $\mathrm{CH}_{2} \mathrm{Cl}_{2}(3 \times 20 \mathrm{~mL})$. The combined organic layers were dried $\left(\mathrm{Na}_{2} \mathrm{SO}_{4}\right)$ and concentrated in vacuo. Flash column chromatography (silica gel, $\mathrm{CH}_{2} \mathrm{Cl}_{2}: \mathrm{MeOH}$ 19:1) afforded amine 5 (1.9 g, 80\% over two steps) as a colorless oil. 5: $R_{\mathrm{f}}=0.50\left(\right.$ silica gel, $\left.\mathrm{CH}_{2} \mathrm{Cl}_{2}: \mathrm{MeOH} 9: 1\right) ;[\alpha]_{\mathrm{D}}{ }^{20}=+9.2\left(\mathrm{c}=1.0, \mathrm{CHCl}_{3}\right) ;{ }^{1} \mathrm{H}$ NMR $(400$ $\left.\mathrm{MHz}, \mathrm{CDCl}_{3}\right) \delta=5.80(\mathrm{ddd}, J=17.3,10.4,7.8 \mathrm{~Hz}, 1 \mathrm{H}), 5.35(\mathrm{~d}, J=17.3 \mathrm{~Hz}, 1 \mathrm{H})$, $5.28(\mathrm{~d}, J=10.4 \mathrm{~Hz}, 1 \mathrm{H}), 4.97(\mathrm{brs}, 2 \mathrm{H}), 3.83(\mathrm{p}, J=6.3 \mathrm{~Hz}, 1 \mathrm{H}), 3.34(\mathrm{t}, J=7.3 \mathrm{~Hz}$, $1 \mathrm{H}), 1.17(\mathrm{~d}, J=6.2 \mathrm{~Hz}, 2 \mathrm{H}), 0.89(\mathrm{~s}, 9 \mathrm{H}), 0.10 \mathrm{ppm}(\mathrm{s}, 6 \mathrm{H}) ;{ }^{13} \mathrm{C} \mathrm{NMR}(100 \mathrm{MHz}$, $\left.\mathrm{CDCl}_{3}\right) \delta=132.7,121.1,69.4,60.6,25.8(3 \mathrm{C}), 20.1,18.0,-4.4,-4.9$ ppm; HRMS (ESI): calcd for $\mathrm{C}_{11} \mathrm{H}_{26} \mathrm{NOSi}^{+}[\mathrm{M}+\mathrm{H}]^{+}$216.1784, found 216.1786. 
ק-Keto amide 3: To a stirred solution of acid 4 (756 mg, $3.1 \mathrm{mmol})$ in $\mathrm{CH}_{2} \mathrm{Cl}_{2}(10 \mathrm{~mL})$<smiles>C=C[C@H](NC(=O)CC(=O)[C@H](C)CC1C(CC[C@](C)(O)C=C)C1(C)C)[C@@H](C)OC(C)(C)C</smiles>
at room temperature were sequentially added Meldrum's acid (6, $893 \mathrm{mg}, 6.2 \mathrm{mmol})$, EDCI (1.2 g, $6.2 \mathrm{mmol})$ and 4-DMAP (756 mg, $6.2 \mathrm{mmol}$ ). The resulting mixture was stirred for 30 min before it was quenched with $\mathrm{NaHSO}_{4}$ (10 mL, sat. aq.). The layers were separated, the aqueous layer was extracted with $\mathrm{CH}_{2} \mathrm{Cl}_{2}(3 \times 5 \mathrm{~mL})$. The combined organic layers were washed with $\mathrm{NaHCO}_{3}(10 \mathrm{~mL}$, sat. aq. $)$ and $\mathrm{H}_{2} \mathrm{O}(10 \mathrm{~mL})$, then dried $\left(\mathrm{Na}_{2} \mathrm{SO}_{4}\right)$ and concentrated in vacuo to afford the crude adduct as a pale yellow oil.

To a stirred solution of the adduct (crude, obtained above) in toluene (20 mL) at room temperature was added amine $5(1.3 \mathrm{~g}, 6.2 \mathrm{mmol})$ and $4 \AA \mathrm{MS}(1.0 \mathrm{~g})$. The mixture was warmed to $80{ }^{\circ} \mathrm{C}$ and stirred for $2 \mathrm{~h}$ before it was cooled to room temperature and filtered through a short pad of celite. The filtrate was collected and concentrated in vacuo. Flash column chromatography (silica gel, hexanes:EtOAc 2:1) afforded $\beta$-keto amide $3(948 \mathrm{mg}, 62 \%)$ as a colorless oil. 3: $R_{\mathrm{f}}=0.50$ (silica gel, hexanes:EtOAc 2:1); $[\alpha]_{\mathrm{D}}{ }^{20}=-38.0\left(\mathrm{c}=1.0, \mathrm{CHCl}_{3}\right) ;{ }^{1} \mathrm{H} \mathrm{NMR}\left(400 \mathrm{MHz}, \mathrm{CDCl}_{3}\right) \delta=7.33(\mathrm{~d}, J=9.1 \mathrm{~Hz}, 1$ H), 5.92-5.76 (m, 2 H), 5.21-5.02 (m, 4 H), 4.41-4.36 (m, $1 \mathrm{H}), 3.96-3.90$ (m, $1 \mathrm{H})$, $3.48(\mathrm{~s}, 2 \mathrm{H}), 2.66-2.60(\mathrm{~m}, 1 \mathrm{H}), 1.61-1.54(\mathrm{~m}, 3 \mathrm{H}), 1.38-1.32(\mathrm{~m}, 2 \mathrm{H}), 1.26(\mathrm{~s}, 3$ H), 1.17-1.12 (m, 1 H), $1.11(\mathrm{~d}, J=7.0 \mathrm{~Hz}, 3 \mathrm{H}), 1.10(\mathrm{~s}, 3 \mathrm{H}), 0.98$ (s, $3 \mathrm{H}), 0.89$ (s, 9 $\mathrm{H}), 0.87(\mathrm{~s}, 3 \mathrm{H}), 0.45-0.38(\mathrm{~m}, 1 \mathrm{H}), 0.36-0.30$ (m, $1 \mathrm{H}), 0.05$ (s, $3 \mathrm{H}), 0.03 \mathrm{ppm}(\mathrm{s}, 3$ $\mathrm{H}) ;{ }^{13} \mathrm{C} \mathrm{NMR}\left(100 \mathrm{MHz}, \mathrm{CDCl}_{3}\right) \delta=210.6,165.5,145.1,136.8,115.6,111.9,73.3$, 70.0, 57.0, 48.2, 47.9, 42.8, 29.3, 28.0, 27.8, 27.0, 25.9 (3C), 24.2, 21.0, 19.1, 18.1, 
17.3, 15.9, 14.8, -4.2, -4.7 ppm; HRMS (ESI): calcd for $\mathrm{C}_{28} \mathrm{H}_{51} \mathrm{NO}_{4} \mathrm{NaSi}^{+}[\mathrm{M}+\mathrm{Na}]^{+}$ 516.3485, found 516.3483.

Macrolactam 17: To a stirred solution of $\beta$-keto amide 3 (887 mg, $1.8 \mathrm{mmol}$ ) in $\mathrm{CH}_{2} \mathrm{Cl}_{2}$<smiles>[3H]NC(=O)CC(=O)[C@@H](C)CC1C(CC[C@](C)(O)/C=C/[C@@H](C(C)OC(C)C)C(C)(C)C)C1(C)C</smiles>
$(600 \mathrm{~mL})$ at room temperature was added Grubbs II cat. (170 mg, $0.2 \mathrm{mmol}$ ). The resulting mixture was warmed to $40{ }^{\circ} \mathrm{C}$ and stirred for $6 \mathrm{~h}$ before it was concentrated in vacuo. Flash column chromatography (silica gel, hexanes:EtOAc 1:1) afforded macrolactam $17(293 \mathrm{mg}, 35 \%)$ as a colorless oil. 17: $R_{\mathrm{f}}$ $=0.40\left(\right.$ silica gel, hexanes:EtOAc 1:1); $[\alpha]_{\mathrm{D}}{ }^{20}=+10.0\left(\mathrm{c}=1.0, \mathrm{CHCl}_{3}\right) ;{ }^{1} \mathrm{H} \mathrm{NMR}(400$ $\left.\mathrm{MHz}, \mathrm{CDCl}_{3}\right) \delta=6.24(\mathrm{~d}, J=8.6 \mathrm{~Hz}, 1 \mathrm{H}), 5.88(\mathrm{~d}, J=16.0 \mathrm{~Hz}, 1 \mathrm{H}), 5.57(\mathrm{dd}, J=$ 16.0, 7.6 Hz, $1 \mathrm{H}), 4.26(\mathrm{t}, J=8.1 \mathrm{~Hz}, 1 \mathrm{H}), 3.96(\mathrm{~m}, 1 \mathrm{H}), 3.48(\mathrm{~d}, J=13.2 \mathrm{~Hz}, 1 \mathrm{H})$, $3.25(\mathrm{~d}, J=13.2 \mathrm{~Hz}, 1 \mathrm{H}), 2.42-2.41(\mathrm{~m}, 1 \mathrm{H}), 1.73-1.65(\mathrm{~m}, 1 \mathrm{H}), 1.47-1.40(\mathrm{~m}, 2 \mathrm{H})$, 1.36-1.29 (m, $2 \mathrm{H}), 1.20$ (s, $3 \mathrm{H}), 1.19$ (d, $J=6.7 \mathrm{~Hz}, 3 \mathrm{H}), 1.13(\mathrm{~d}, J=6.2 \mathrm{~Hz}, 3 \mathrm{H})$, $1.02(\mathrm{~s}, 3 \mathrm{H}), 0.97$ (s, $3 \mathrm{H}), 0.92(\mathrm{~s}, 9 \mathrm{H}), 0.77-0.68(\mathrm{~m}, 1 \mathrm{H}), 0.64-0.58(\mathrm{~m}, 1 \mathrm{H}), 0.54$ $0.48(\mathrm{~m}, 1 \mathrm{H}), 0.09(\mathrm{~s}, 3 \mathrm{H}), 0.08 \mathrm{ppm}(\mathrm{s}, 3 \mathrm{H}) ;{ }^{13} \mathrm{C} \mathrm{NMR}\left(150 \mathrm{MHz}, \mathrm{CDCl}_{3}\right) \delta=207.7$ 164.5, 141.1, 127.4, 73.6, 69.3, 56.2, 49.1, 48.9, 42.8, 29.2, 27.6, 26.7, 25.9 (3C), 24.1, 22.6, 21.3, 19.9, 18.1, 17.7, 15.0, 14.2, -3.9, -4.9 ppm; HRMS (ESI): calcd for $\mathrm{C}_{26} \mathrm{H}_{47} \mathrm{NO}_{4} \mathrm{NaSi}^{+}[\mathrm{M}+\mathrm{Na}]^{+}$488.3172, found 488.3175.

Tetrazolyl sulfone 18: To a stirred solution of tert-butyl $(R)$-(1-hydroxybut-3-en-2-

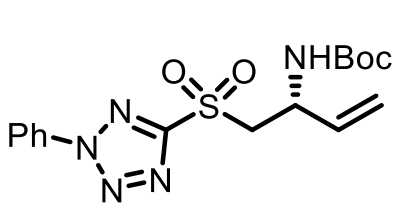

18 yl)carbamate ${ }^{4}(1.8 \mathrm{~g}, 9.6 \mathrm{mmol})$ in $\mathrm{THF}(10 \mathrm{~mL})$ at $0{ }^{\circ} \mathrm{C}$ were sequentially added $\mathrm{PPh}_{3}(2.6 \mathrm{~g}, 9.9 \mathrm{mmol})$, PMTA (1.8 g, $10.1 \mathrm{mmol})$ and DEAD (1.7 $\mathrm{g}, 9.8 \mathrm{mmol})$. The resulting 
mixture was stirred for $5 \mathrm{~h}$ before it was quenched with $\mathrm{H}_{2} \mathrm{O}(10 \mathrm{~mL})$. The layers were separated, the aqueous layer was extracted with $\mathrm{Et}_{2} \mathrm{O}(3 \times 5 \mathrm{~mL})$. The combined organic layers were dried $\left(\mathrm{Na}_{2} \mathrm{SO}_{4}\right)$ and concentrated in vacuo to afford the crude tetrazolyl sulfide as a white amorphous solid.

To a stirred solution of the tetrazolyl sulfide (crude, obtained above) in EtOH (5 $\mathrm{mL})$ at room temperature were added ammonium molybdate tetrahydrate $(2.5 \mathrm{~g}, 2.0$ mmol) and $\mathrm{H}_{2} \mathrm{O}_{2}(5.0 \mathrm{~mL}, 8.8 \mathrm{M}$ aq., $44.0 \mathrm{mmol})$. The resulting mixture was stirred for $1 \mathrm{~h}$ before it was quenched with $\mathrm{Na}_{2} \mathrm{~S}_{2} \mathrm{O}_{3}(10 \mathrm{~mL}$, sat. aq.). The layers were separated, and the aqueous layer was extracted with $\mathrm{CH}_{2} \mathrm{Cl}_{2}(3 \times 5 \mathrm{~mL})$. The combined organic layers were dried $\left(\mathrm{Na}_{2} \mathrm{SO}_{4}\right)$ and concentrated in vacuo. Flash column chromatography (silica gel, hexanes:EtOAc 4:1) afforded tetrazolyl sulfone $\mathbf{1 8}(3.1 \mathrm{~g}, 85 \%)$ as a white amorphous solid. 18: $R_{\mathrm{f}}=0.50$ (silica gel, hexanes:EtOAc $\left.2: 1\right) ;[\alpha]_{\mathrm{D}}{ }^{20}=+32.5(\mathrm{c}=1.0$, $\left.\mathrm{CHCl}_{3}\right) ;{ }^{1} \mathrm{H} \mathrm{NMR}\left(400 \mathrm{MHz}, \mathrm{CDCl}_{3}\right) \delta=7.64-7.54(\mathrm{~m}, 5 \mathrm{H}), 5.84(\mathrm{ddd}, J=17.0,10.4$, $5.7 \mathrm{~Hz}, 1 \mathrm{H}), 5.26(\mathrm{dd}, J=17.0,1.5 \mathrm{~Hz}, 1 \mathrm{H}), 5.18(\mathrm{dd}, J=10.4,1.5 \mathrm{~Hz}, 1 \mathrm{H}), 5.12$ $(\mathrm{ddd}, J=8.8 \mathrm{~Hz}, 1 \mathrm{H}), 4.78(\mathrm{p}, J=6.4 \mathrm{~Hz}, 1 \mathrm{H}), 4.13(\mathrm{dd}, J=15.0,7.5 \mathrm{~Hz}, 1 \mathrm{H}), 3.97$ $(\mathrm{dd}, J=15.0,4.7 \mathrm{~Hz}, 1 \mathrm{H}), 1.36 \mathrm{ppm}(\mathrm{s}, 9 \mathrm{H}) ;{ }^{13} \mathrm{C} \mathrm{NMR}\left(100 \mathrm{MHz}, \mathrm{CDCl}_{3}\right) \delta=154.6$, 153.8, 134.6, 133.0, 131.6, 129.7 (2C), 125.4 (2C), 117.5, 80.4, 59.4, 48.7, $28.3 \mathrm{ppm}$ (3C); HRMS (ESI): calcd for $\mathrm{C}_{16} \mathrm{H}_{21} \mathrm{~N}_{5} \mathrm{O}_{4} \mathrm{NaS}^{+}[\mathrm{M}+\mathrm{Na}]^{+}$402.1212, found 402.1219.

Phosphonate 19: To a stirred solution of diethyl (2-oxoethyl)phosphonate ${ }^{5}$ (3.6 g, 20.0<smiles>CCOP(=O)(CC=NS(=O)[13C](C)(C)C)OCC</smiles>
mmol) in $\mathrm{CH}_{2} \mathrm{Cl}_{2}(10 \mathrm{~mL})$ at room temperature were sequentially added PPTS (1.0 g, $4.0 \mathrm{mmol}), \mathrm{MgSO}_{4}(6.0 \mathrm{~g}$, $50.0 \mathrm{mmol})$ and $(S)$-(-)-tert-butansulfinamide $(2.4 \mathrm{~g}, 20.0$ 
mmol). The resulting mixture was stirred for $2 \mathrm{~h}$ before it was filtered through a short pad of celite. The filtrate was collected and concentrated in vacuo. Flash column chromatography (silica gel, $\left.\mathrm{CH}_{2} \mathrm{Cl}_{2}: \mathrm{MeOH} 19: 1\right)$ afforded phosphonate 19 (4.8 g, 85\%) as a colorless oil. 19: $R_{\mathrm{f}}=0.50$ (silica gel, $\left.\mathrm{CH}_{2} \mathrm{Cl}_{2}: \mathrm{MeOH} 9: 1\right) ;[\alpha]_{\mathrm{D}}{ }^{20}=+84.0(\mathrm{c}=1.0$, $\left.\mathrm{CHCl}_{3}\right) ;{ }^{1} \mathrm{H} \mathrm{NMR}\left(400 \mathrm{MHz}, \mathrm{CDCl}_{3}\right) \delta=8.01-7.97(\mathrm{~m}, 1 \mathrm{H}), 4.20-4.11(\mathrm{~m}, 4 \mathrm{H}) \mathrm{ppm}$, 3.18-3.11 (m, $2 \mathrm{H}), 1.35-1.32(\mathrm{~m}, 6 \mathrm{H}), 1.22 \mathrm{ppm}(\mathrm{s}, 9 \mathrm{H}) ;{ }^{13} \mathrm{C} \mathrm{NMR}\left(100 \mathrm{MHz}, \mathrm{CDCl}_{3}\right)$ $\delta=160.3,62.6,57.5,35.8,34.5,22.5$ (3C), 16.5 ppm (2C); HRMS (ESI): calcd for $\mathrm{C}_{10} \mathrm{H}_{22} \mathrm{NO}_{4} \mathrm{NaSP}^{+}[\mathrm{M}+\mathrm{Na}]^{+}$306.0905, found 306.0901.

Imide 20: To a stirred solution of phosphonate 19 (4.2 g, $14.8 \mathrm{mmol})$ in THF (20 mL)<smiles>COC(=O)[C@H](C)CC1C(CC[C@](C)(/C=C/C=N\S(=O)C(C)(C)C)OC(C)(C)C)C1(C)C</smiles>

20

at $0{ }^{\circ} \mathrm{C}$ was added LiHMDS (11.1 $\mathrm{mL}, 1.0 \mathrm{M}$ in THF, $11.1 \mathrm{mmol})$. The resulting mixture was stirred at $0{ }^{\circ} \mathrm{C}$ for $30 \mathrm{~min}$ before it was added to a solution of aldehyde 16 (2.0 g, $7.4 \mathrm{mmol})$ in THF (20 mL). The reaction was stirred at $0{ }^{\circ} \mathrm{C}$ for further $10 \mathrm{~h}$ before it was quenched with $\mathrm{NH}_{4} \mathrm{Cl}$ (40 mL, sat. aq.). The layers were separated, and the aqueous layer was extracted with $\mathrm{Et}_{2} \mathrm{O}(3 \times 20 \mathrm{~mL})$. The combined organic layers were dried $\left(\mathrm{Na}_{2} \mathrm{SO}_{4}\right)$ and concentrated in vacuo to afford the crude alkene as a pale yellow oil.

To a stirred solution of the alkene (crude, obtained above) in $\mathrm{CH}_{2} \mathrm{Cl}_{2}(50 \mathrm{~mL})$ at 0 ${ }^{\circ} \mathrm{C}$ were added 2,6-lutidine (2.6 mL, $\left.22.2 \mathrm{mmol}\right)$ and TBSOTf $(2.6 \mathrm{~mL}, 11.1 \mathrm{mmol})$. The mixture was stirred at $0{ }^{\circ} \mathrm{C}$ for $1 \mathrm{~h}$ before it was quenched with $\mathrm{NaHCO}_{3}(50 \mathrm{~mL}$, sat. aq.). The layers were separated, and the aqueous layer was extracted with $\mathrm{CH}_{2} \mathrm{Cl}_{2}$ $(3 \times 20 \mathrm{~mL})$. The combined organic layers were dried $\left(\mathrm{Na}_{2} \mathrm{SO}_{4}\right)$ and concentrated in 
vacuo. Flash column chromatography (silica gel, hexanes:EtOAc 9:1) afforded imide $20(2.7 \mathrm{~g}, 70 \%)$ as a colorless oil. 20: $R_{\mathrm{f}}=0.45$ (silica gel, hexanes:EtOAc 4:1); $[\alpha]_{\mathrm{D}}{ }^{20}$ $=+163.4\left(\mathrm{c}=1.0, \mathrm{CHCl}_{3}\right) ;{ }^{1} \mathrm{H} \mathrm{NMR}\left(400 \mathrm{MHz}, \mathrm{CDCl}_{3}\right) \delta=8.20(\mathrm{dd}, J=6.7,2.1 \mathrm{~Hz}$, $1 \mathrm{H}), 6.50-6.48$ (m, $2 \mathrm{H}), 3.66$ (s, $3 \mathrm{H}), 2.48-2.39$ (m, $1 \mathrm{H}), 1.63-1.50$ (m, $3 \mathrm{H}), 1.38$ (s, $3 \mathrm{H}), 1.39-1.32(\mathrm{~m}, 2 \mathrm{H}), 1.21(\mathrm{~s}, 9 \mathrm{H}), 1.15$ (d, J = 7.0 Hz, $3 \mathrm{H}), 1.14-1.09$ (m, 1 H), 0.99 (s, 3 H), 0.90 (s, 9 H), 0.85 (s, 3 H), 0.40-0.34 (m, 2 H), 0.09 (s, 3 H), 0.06 $\operatorname{ppm}(\mathrm{s}, 3 \mathrm{H}) ;{ }^{13} \mathrm{C} \mathrm{NMR}\left(100 \mathrm{MHz}, \mathrm{CDCl}_{3}\right) \delta=177.3,164.0,157.7,125.6,75.6,57.4$, 51.6, 44.1, 40.3, 29.4, 29.0, 27.7, 26.8, 26.1 (3C), 24.4, 22.6 (3C), 19.2, 18.5, 17.2, 17.1, 14.8, -1.9 ppm (2C); HRMS (ESI): calcd for $\mathrm{C}_{27} \mathrm{H}_{51} \mathrm{NO}_{4} \mathrm{NaSiS}^{+}[\mathrm{M}+\mathrm{Na}]^{+}$536.3206, found 536.3210.

Sulfamide 21: To a stirred solution of imide 20 (2.5 g, $4.9 \mathrm{mmol})$ in THF (30 mL) at -<smiles>C=C[C@H](/C=C/[C@](C)(CCC1C(C[C@H](C)C(=O)OC)C1(C)C)C(C)(C)C)NS(=O)C(C)(C)C</smiles>

21

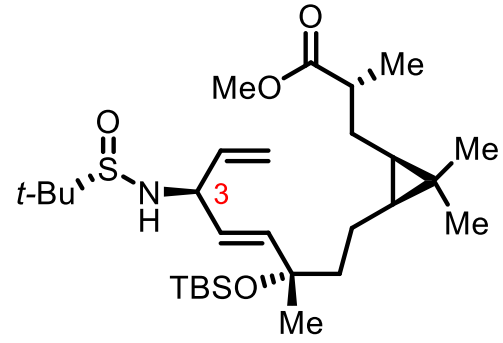

3-epi-21 $60 \quad{ }^{\circ} \mathrm{C}$ was added vinylMgBr $(17.1 \mathrm{~mL}$, $1.0 \mathrm{M}$ in $\mathrm{THF}, 17.1$ mmol). The resulting mixture was stirred at $-60{ }^{\circ} \mathrm{C}$ for $10 \mathrm{~h}$ before it was quenched with $\mathrm{NH}_{4} \mathrm{Cl}(50 \mathrm{~mL}$, sat. aq. $)$. The layers were separated, and the aqueous layer was extracted with $\mathrm{Et}_{2} \mathrm{O}(3 \times 20$ $\mathrm{mL})$. The combined organic layers were dried $\left(\mathrm{Na}_{2} \mathrm{SO}_{4}\right)$ and concentrated in vacuo. Flash column chromatography (silica gel, hexanes:EtOAc 4:1) afforded sulfamide 21 $(1.6 \mathrm{~g}, 61 \%)$ as a colorless oil, along with sulfamide 3 -epi-21 (451 mg, 17\%) as a colorless oil. 21: $R_{\mathrm{f}}=0.40$ (silica gel, hexanes:EtOAc $\left.2: 1\right) ;[\alpha]_{\mathrm{D}}^{20}=+34.0(\mathrm{c}=1.0$, $\left.\mathrm{CHCl}_{3}\right) ;{ }^{1} \mathrm{H} \mathrm{NMR}\left(400 \mathrm{MHz}, \mathrm{CDCl}_{3}\right) \delta=5.84(\mathrm{ddd}, J=17.0,10.1,6.7 \mathrm{~Hz}, 1 \mathrm{H}), 5.67$ 
$(\mathrm{d}, J=15.5 \mathrm{~Hz}, 1 \mathrm{H}), 5.45(\mathrm{dd}, J=15.5,7.4 \mathrm{~Hz}, 1 \mathrm{H}), 5.24(\mathrm{~d}, J=17.0 \mathrm{~Hz}, 1 \mathrm{H}), 5.14$ $(\mathrm{d}, J=10.1 \mathrm{~Hz}, 1 \mathrm{H}), 4.37(\mathrm{dd}, J=7.0,3.4 \mathrm{~Hz}, 1 \mathrm{H}), 3.66(\mathrm{~s}, 3 \mathrm{H}), 3.19(\mathrm{~d}, J=3.4 \mathrm{~Hz}$, $1 \mathrm{H}), 2.46-2.41(\mathrm{~m}, 1 \mathrm{H}), 1.58-1.43(\mathrm{~m}, 3 \mathrm{H}), 1.39-1.26$ (m, $2 \mathrm{H}), 1.29$ (s, $3 \mathrm{H}), 1.20$ (s, $9 \mathrm{H}), 1.14$ (d, J = 7.0 Hz, $3 \mathrm{H}), 1.14-1.09$ (m, $1 \mathrm{H}), 0.98$ (s, $3 \mathrm{H}), 0.86$ (s, $9 \mathrm{H}), 0.85$ (s, $3 \mathrm{H}), 0.40-0.33(\mathrm{~m}, 2 \mathrm{H}), 0.05(\mathrm{~s}, 3 \mathrm{H}), 0.03 \mathrm{ppm}(\mathrm{s}, 3 \mathrm{H}) ;{ }^{13} \mathrm{C}$ NMR (100 MHz, $\left.\mathrm{CDCl}_{3}\right) \delta=177.4,141.0,138.6,126.5,116.5,75.3,59.6,55.6,51.6,44.6,40.3,29.4$, 29.0, 28.0, 26.9, 26.1 (3C), 24.4, 22.7 (3C), 19.2, 18.5, 17.1 (2C), 14.8, -1.8, -1.9 ppm; HRMS (ESI): calcd for $\mathrm{C}_{29} \mathrm{H}_{55} \mathrm{NO}_{4} \mathrm{NaSiS}^{+}[\mathrm{M}+\mathrm{Na}]^{+}$564.3519, found 564.3518.

3-epi-21: $R_{\mathrm{f}}=0.35$ (silica gel, hexanes:EtOAc 2:1); $[\alpha]_{\mathrm{D}}{ }^{20}=+31.6\left(\mathrm{c}=1.0, \mathrm{CHCl}_{3}\right)$; ${ }^{1} \mathrm{H}$ NMR $\left(400 \mathrm{MHz}, \mathrm{CDCl}_{3}\right) \delta=5.76-5.68(\mathrm{~m}, 2 \mathrm{H}), 5.58(\mathrm{dd}, J=15.7,6.5 \mathrm{~Hz}, 1 \mathrm{H})$, $5.27(\mathrm{~d}, J=16.8 \mathrm{~Hz}, 1 \mathrm{H}), 5.19(\mathrm{~d}, J=10.2 \mathrm{~Hz}, 1 \mathrm{H}), 4.37(\mathrm{dd}, J=7.0,3.8 \mathrm{~Hz}, 1 \mathrm{H})$, 3.67 (s, $3 \mathrm{H}), 3.15$ (d, J =3.8 Hz, $1 \mathrm{H}), 2.46-2.41$ (m, $1 \mathrm{H}), 1.69-1.48$ (m, $1 \mathrm{H}), 1.56-$ $1.44(\mathrm{~m}, 3 \mathrm{H}), 1.40-1.33(\mathrm{~m}, 1 \mathrm{H}), 1.28(\mathrm{~s}, 3 \mathrm{H}), 1.21(\mathrm{~s}, 9 \mathrm{H}), 1.15(\mathrm{~d}, J=7.0 \mathrm{~Hz}, 3 \mathrm{H})$, 1.14-1.11 (m, $1 \mathrm{H}), 0.98$ (s, $3 \mathrm{H}), 0.86$ (s, $9 \mathrm{H}), 0.86$ (s, $3 \mathrm{H}), 0.38-0.34$ (m, $2 \mathrm{H}), 0.05$ (s, $3 \mathrm{H}), 0.04 \mathrm{ppm}(\mathrm{s}, 3 \mathrm{H}) ;{ }^{13} \mathrm{C} \mathrm{NMR}\left(100 \mathrm{MHz}, \mathrm{CDCl}_{3}\right) \delta=177.4,140.5,137.8,127.3$, $117.4,75.1,59.4,55.7,51.6,44.5,40.3,29.4,29.0,27.7,26.9,26.1(3 C), 24.4,22.7$ (3C), 19.2, 18.4, 17.2, 17.1, 14.9, $-1.7,-1.8$ ppm; HRMS (ESI): calcd for $\mathrm{C}_{29} \mathrm{H}_{55} \mathrm{NO}_{4} \mathrm{NaSiS}^{+}[\mathrm{M}+\mathrm{Na}]^{+}$564.3519, found 564.3515.

Acid 22: To a stirred solution of sulfamide 21 (1.5 g, $2.8 \mathrm{mmol})$ in $\mathrm{Et}_{2} \mathrm{O}(20 \mathrm{~mL})$ at room temperature was added $\mathrm{HCl}\left(5.0 \mathrm{~mL}, 1.0 \mathrm{M}\right.$ in $\left.\mathrm{Et}_{2} \mathrm{O}, 5.0 \mathrm{mmol}\right)$. The resulting mixture was stirred for 10 min before it was diluted with $\mathrm{Et}_{2} \mathrm{O}(20 \mathrm{~mL})$ and $\mathrm{H}_{2} \mathrm{O}(50$ $\mathrm{mL})$. The layers were separated, and the aqueous layer was extracted with $\mathrm{Et}_{2} \mathrm{O}(3 \times 20$ 


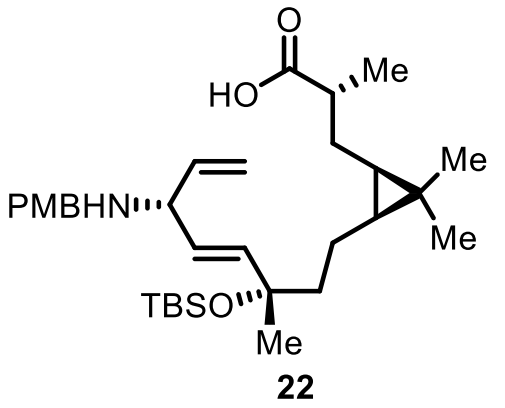

$\mathrm{mL})$. The combined organic layers were dried $\left(\mathrm{Na}_{2} \mathrm{SO}_{4}\right)$

and concentrated in vacuo to afford a solution $(20 \mathrm{~mL})$, which was added $\mathrm{MeOH}(20 \mathrm{~mL})$. The resulting mixture was stirred at room temperature for $30 \mathrm{~min}$

before it was quenched with $\mathrm{NaHCO}_{3}(50 \mathrm{~mL}$, sat. aq.). The layers were separated, and the aqueous layer was extracted with $\mathrm{Et}_{2} \mathrm{O}(3 \times 20 \mathrm{~mL})$. The combined organic layers were dried $\left(\mathrm{Na}_{2} \mathrm{SO}_{4}\right)$ and concentrated in vacuo to afford the crude primary amine as a pale yellow oil.

To a stirred solution of the primary amine (crude, obtained above) in $\mathrm{MeOH}$ (20 $\mathrm{mL})$ at room temperature was added PMPCHO $(1.7 \mathrm{~mL}, 14.0 \mathrm{mmol})$. The resulting mixture was stirred for $3 \mathrm{~h}$ before $\mathrm{NaBH}_{4}(550 \mathrm{mg}, 14.5 \mathrm{mmol})$ was slowly added. The resulting mixture was stirred for further $30 \mathrm{~min}$ before it was added $\mathrm{LiOH}(7.0 \mathrm{~mL}, 4.0$ $\mathrm{M}$ aq., $28.0 \mathrm{mmol}$ ). The reaction was warmed to $60{ }^{\circ} \mathrm{C}$ and stirred for an additional $6 \mathrm{~h}$ before it was acidified with $\mathrm{HCl}(40 \mathrm{~mL}, 1.0 \mathrm{M}$ aq.). The layers were separated, and the aqueous layer was extracted with $\mathrm{Et}_{2} \mathrm{O}(3 \times 20 \mathrm{~mL})$. The combined organic layers were dried $\left(\mathrm{Na}_{2} \mathrm{SO}_{4}\right)$ and concentrated in vacuo. Flash column chromatography (silica gel, $\left.\mathrm{CH}_{2} \mathrm{Cl}_{2}: \mathrm{MeOH} 19: 1\right)$ afforded acid 22 (1.2 g, 82\% over two steps) as a white amorphous solid. 22: $R_{\mathrm{f}}=0.50\left(\right.$ silica gel, EtOAc); $[\alpha]_{\mathrm{D}}{ }^{20}=+22.0\left(\mathrm{c}=1.0, \mathrm{CHCl}_{3}\right) ;{ }^{1} \mathrm{H} \mathrm{NMR}(400$ $\left.\mathrm{MHz}, \mathrm{CDCl}_{3}\right) \delta=7.71(\mathrm{brs}, 1 \mathrm{H}), 7.33(\mathrm{~d}, J=8.6 \mathrm{~Hz}, 2 \mathrm{H}), 6.77(\mathrm{~d}, J=8.6 \mathrm{~Hz}, 2 \mathrm{H})$, $5.94(\mathrm{ddd}, J=17.3,10.4,7.1 \mathrm{~Hz}, 1 \mathrm{H}), 5.70-5.50$ (m, $2 \mathrm{H}), 5.21-5.16$ (m, 2 H), 3.85$3.74(\mathrm{~m}, 3 \mathrm{H}), 3.67(\mathrm{~s}, 3 \mathrm{H}), 2.36-2.32(\mathrm{~m}, 1 \mathrm{H}), 1.56-1.36(\mathrm{~m}, 3 \mathrm{H}), 1.33-1.26(\mathrm{~m}, 1$ H), 1.22 (s, $3 \mathrm{H}), 1.19-1.12(\mathrm{~m}, 2 \mathrm{H}), 1.05$ (d, $J=7.0 \mathrm{~Hz}, 3 \mathrm{H}), 0.91$ (s, $3 \mathrm{H}), 0.79$ (s, 9 
$\mathrm{H}), 0.76(\mathrm{~s}, 3 \mathrm{H}), 0.38-0.26$ (m, $2 \mathrm{H}),-0.03$ (s, $3 \mathrm{H}),-0.04 \mathrm{ppm}(\mathrm{s}, 3 \mathrm{H}) ;{ }^{13} \mathrm{C}$ NMR $(100$ $\left.\mathrm{MHz}, \mathrm{CDCl}_{3}\right) \delta=181.3,159.7,143.3,135.2,131.1(2 \mathrm{C}), 126.1,124.0,119.5,114.3$ (2C), 75.5, 61.3, 55.4, 48.4, 44.2, 39.4, 29.9, 29.6, 28.5, 27.0, 26.1 (3C), 23.2, 20.0, 18.4 17.2, 15.8, 14.8, -1.7, -1.9 ppm; HRMS (ESI): calcd for $\mathrm{C}_{32} \mathrm{H}_{54} \mathrm{NO}_{4} \mathrm{Si}^{+}[\mathrm{M}+\mathrm{H}]^{+}$ 544.3822, found 544.3825.

Macrolactam 24: To a stirred solution of acid 22 (1.2 g, $2.2 \mathrm{mmol})$ in $\mathrm{CH}_{2} \mathrm{Cl}_{2}(10 \mathrm{~mL})$<smiles>C=C[C@H](/C=C/[C@@H](CCC1C(C[C@H](C)C(=O)CC(C)(C)C)C1(C)C)C(=O)O)C(C)(C)C</smiles>
at room temperature were sequentially added Meldrum's acid (6, $634 \mathrm{mg}, 4.4 \mathrm{mmol})$, EDCI (845 mg, $4.4 \mathrm{mmol})$ and 4-DMAP (537 mg, $4.4 \mathrm{mmol})$. The resulting mixture was stirred for $2 \mathrm{~h}$ before it was quenched with $\mathrm{NaHSO}_{4}(10 \mathrm{~mL}$, sat. aq.). The layers were separated, the aqueous layer was extracted with $\mathrm{CH}_{2} \mathrm{Cl}_{2}(3 \times$ $10 \mathrm{~mL})$. The combined organic layers were washed with $\mathrm{NaHCO}_{3}(10 \mathrm{~mL}$, sat. aq.) and $\mathrm{H}_{2} \mathrm{O}(10 \mathrm{~mL})$, then dried $\left(\mathrm{Na}_{2} \mathrm{SO}_{4}\right)$ and concentrated in vacuo to afford the crude adduct as a pale yellow oil.

To a stirred solution of the adduct (crude, obtained above) in toluene (100 mL) at room temperature was added $4 \AA \mathrm{MS}(1.0 \mathrm{~g})$. The mixture was warmed to $110^{\circ} \mathrm{C}$ and stirred for $2 \mathrm{~h}$ before it was cooled to room temperature and filtered through a short pad of celite. The filtrate was collected and concentrated in vacuo. Flash column chromatography (silica gel, hexanes:EtOAc 9:1) afforded macrolactam 24 (836 mg, 67\%) as a white amorphous solid. 24: $R_{\mathrm{f}}=0.50$ (silica gel, hexanes:EtOAc 4:1); $[\alpha]_{\mathrm{D}}{ }^{20}$ $=+11.0\left(\mathrm{c}=1.0, \mathrm{CHCl}_{3}\right) ;{ }^{1} \mathrm{H} \mathrm{NMR}\left(600 \mathrm{MHz}, \mathrm{CD}_{3} \mathrm{CN}, 60{ }^{\circ} \mathrm{C}\right.$, ca. a 1:1 mixture of atropisomers) $\delta=7.30-7.26(\mathrm{~m}, 2 \mathrm{H}), 7.01-6.93(\mathrm{~m}, 2 \mathrm{H}), 6.10-6.05(\mathrm{~m}, 1 \mathrm{H}), 5.88-$ 
$5.85(\mathrm{~m}, 1 \mathrm{H}), 5.77-5.70(\mathrm{~m}, 1 \mathrm{H}), 5.32-5.26(\mathrm{~m}, 2 \mathrm{H}), 5.05$ (brs, 0.5 H), 4.65-4.57 (m, $2 \mathrm{H}), 4.20$ (brs, $0.5 \mathrm{H}), 3.90-3.86$ (m, $3 \mathrm{H}), 3.68$ (brs, $0.5 \mathrm{H}), 3.28$ (brs, $0.5 \mathrm{H}), 2.75$ (brs, $0.5 \mathrm{H}), 2.50$ (brs, $0.5 \mathrm{H}), 1.85$ (brs, $1 \mathrm{H}), 1.62-1.52(\mathrm{~m}, 2 \mathrm{H}), 1.53-1.49$ (m, $1 \mathrm{H})$, 1.45-1.42 (m, $1 \mathrm{H}), 1.38-1.34(\mathrm{~m}, 2 \mathrm{H}), 1.18(\mathrm{~d}, J=7.0 \mathrm{~Hz}, 3 \mathrm{H}), 1.15$ (s, $3 \mathrm{H}), 1.13$ (s, $3 \mathrm{H}), 1.01(\mathrm{~s}, 3 \mathrm{H}), 0.98$ (s, $9 \mathrm{H}), 0.71-0.61(\mathrm{~m}, 2 \mathrm{H}), 0.16 \mathrm{ppm}(\mathrm{s}, 6 \mathrm{H}) ;{ }^{13} \mathrm{C} \mathrm{NMR}$ $\left(150 \mathrm{MHz}, \mathrm{CD}_{3} \mathrm{CN}, 60{ }^{\circ} \mathrm{C}\right.$, ca. a 1:1 mixture of atropisomers) $\delta=209.1,207.0,169.3$, 168.2, 160.6, 160.2, 142.5, 137.7 (2C), 132.7, 131.1, 130.6 (2C), 129.7, 126.4, 120.9, 119.0, 115.5 (2C), 115.1 (2C), 105.8, 102.2, 98.0, 77.4, 77.3, 63.6, 57.0, 56.8, 56.7, 56.4 (3C), 48.5, 47.4, 46.6, 45.3, 45.1, 45.0, 30.6, 29.7, 29.4, 28.6, 28.3, 28.2, 28.0, 27.7, 26.8, 26.6 (3C), 25.7, 25.5, 24.4, 23.9, 21.0, 20.9, 19.0, 18.5, 16.0, 15.9, 15.8, 14.7, 14.3, -1.3 (4C) ppm; HRMS (ESI): calcd for $\mathrm{C}_{34} \mathrm{H}_{53} \mathrm{NO}_{4} \mathrm{NaSi}^{+}\left[\mathrm{M}+\mathrm{Na}^{+}\right.$ 590.3642 , found 590.3645 .

Tricycle 25: To a stirred solution of macrolactam $24(226 \mathrm{mg}, 0.4 \mathrm{mmol})$ in toluene (80

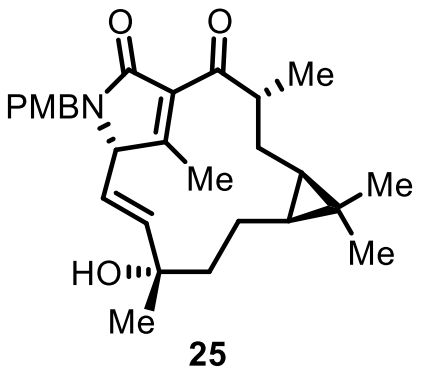
$\mathrm{mL})$ at room temperature was added $\mathrm{Pd}(\mathrm{OAc})_{2}(99 \mathrm{mg}, 0.44$ mmol). The reaction was warmed to $75^{\circ} \mathrm{C}$ and stirred for 2 $\mathrm{h}$ before it was cooled to room temperature and added $\mathrm{MeOH}(80 \mathrm{~mL})$ followed by $\mathrm{HCl}(80 \mathrm{~mL}, 2.0 \mathrm{M}$ aq., 0.16 mol). The resulting mixture was stirred for further $6 \mathrm{~h}$ before it was diluted with $\mathrm{CH}_{2} \mathrm{Cl}_{2}$ (50 mL). The layers were separated, and the aqueous layer was extracted with $\mathrm{CH}_{2} \mathrm{Cl}_{2}$ $(3 \times 30 \mathrm{~mL})$. The combined organic layers were dried $\left(\mathrm{Na}_{2} \mathrm{SO}_{4}\right)$ and concentrated in vacuo. Flash column chromatography (silica gel, hexanes:EtOAc 1:1) afforded tricycle $25(118 \mathrm{mg}, 65 \%)$ as a colorless oil. 25: $R_{\mathrm{f}}=0.40$ (silica gel, hexanes:EtOAc 1:1); $[\alpha]_{\mathrm{D}}{ }^{20}$ 
$=+81.2\left(\mathrm{c}=1.0, \mathrm{CHCl}_{3}\right) ;{ }^{1} \mathrm{H} \mathrm{NMR}\left(400 \mathrm{MHz}, \mathrm{CDCl}_{3}\right) \delta=7.12(\mathrm{~d}, J=6.6 \mathrm{~Hz}, 2 \mathrm{H})$, $6.81(\mathrm{~d}, J=6.6 \mathrm{~Hz}, 2 \mathrm{H}), 5.63(\mathrm{dd}, J=16.0,2.2 \mathrm{~Hz}, 1 \mathrm{H}), 5.53(\mathrm{~d}, J=16.0 \mathrm{~Hz}, 1 \mathrm{H})$, $5.14(\mathrm{~d}, J=14.7 \mathrm{~Hz}, 1 \mathrm{H}), 4.20(\mathrm{~d}, J=2.2 \mathrm{~Hz}, 1 \mathrm{H}), 3.81(\mathrm{~d}, J=14.7 \mathrm{~Hz}, 1 \mathrm{H}), 3.77(\mathrm{~s}$, $3 \mathrm{H}), 2.47-2.40$ (m, $1 \mathrm{H}), 1.93$ (s, $3 \mathrm{H}), 1.87-1.77$ (m, $3 \mathrm{H}), 1.60-1.52$ (m, $1 \mathrm{H}), 1.48-$ $1.40(\mathrm{~m}, 1 \mathrm{H}), 1.31$ (s, $3 \mathrm{H}), 1.29$ (d, J = 7.0 Hz, $3 \mathrm{H}), 0.98$ (s, $3 \mathrm{H}), 0.87$ (s, $3 \mathrm{H}), 0.54$ $0.48(\mathrm{~m}, 2 \mathrm{H}), 0.44-0.36 \mathrm{ppm}(\mathrm{m}, 1 \mathrm{H}) ;{ }^{13} \mathrm{C} \mathrm{NMR}\left(100 \mathrm{MHz}, \mathrm{CDCl}_{3}\right) \delta=205.9,168.2$, $159.3,154.9,139.3,132.3,129.8(2 \mathrm{C}), 129.0,122.2,114.2(2 \mathrm{C}), 73.7,62.4,55.4,47.9$, 43.5, 42.5, 29.3, 28.4, 26.9, 22.2, 21.7, 20.0, 18.4, 15.5, 14.5, 14.0 ppm; HRMS (ESI): calcd for $\mathrm{C}_{28} \mathrm{H}_{37} \mathrm{NO}_{4} \mathrm{Na}^{+}[\mathrm{M}+\mathrm{Na}]^{+}$474.2620, found 474.2622.

Tricycle 26: To a stirred solution of macrolactam 24 (113 mg, $0.2 \mathrm{mmol})$ in toluene (40

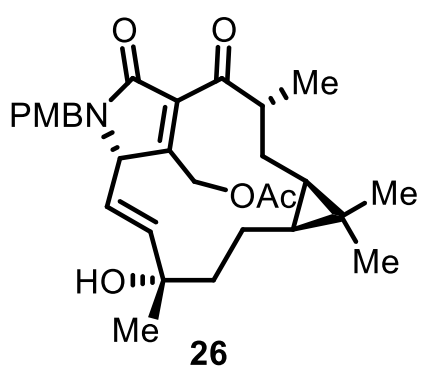
$\mathrm{mL})$ at room temperature was added $\mathrm{Pd}(\mathrm{OAc})_{2}(90 \mathrm{mg}, 0.4$ mmol). The reaction was warmed to $75^{\circ} \mathrm{C}$ and stirred for 2 $\mathrm{h}$ before it was cooled to room temperature and added $\mathrm{MeOH}(40 \mathrm{~mL})$ followed by $\mathrm{HCl}(40 \mathrm{~mL}, 2.0 \mathrm{M}$ aq., 80.0 mmol). The resulting mixture was stirred for further $6 \mathrm{~h}$ before it was diluted with $\mathrm{CH}_{2} \mathrm{Cl}_{2}(30 \mathrm{~mL})$. The layers were separated, and the aqueous layer was extracted with $\mathrm{CH}_{2} \mathrm{Cl}_{2}(3 \times 20 \mathrm{~mL})$. The combined organic layers were dried $\left(\mathrm{Na}_{2} \mathrm{SO}_{4}\right)$ and concentrated in vacuo. Flash column chromatography (silica gel, hexanes:EtOAc 1:1) afforded tricycle $\mathbf{2 5}$ (9 mg, 10\%) as a colorless oil, along with tricycle $\mathbf{2 6}$ (41 $\mathrm{mg}, \mathbf{4 0 \%}$ ) as a colorless oil. 26: $R_{\mathrm{f}}=0.39$ (silica gel, hexanes:EtOAc $\left.1: 1\right) ;[\alpha]_{\mathrm{D}}{ }^{20}=+55.6(\mathrm{c}=1.0$, $\left.\mathrm{CHCl}_{3}\right) ;{ }^{1} \mathrm{H} \mathrm{NMR}\left(400 \mathrm{MHz}, \mathrm{CDCl}_{3}\right) \delta=7.13(\mathrm{~d}, J=8.5 \mathrm{~Hz}, 2 \mathrm{H}), 6.83(\mathrm{~d}, J=8.5 \mathrm{~Hz}$, $2 \mathrm{H}), 5.57$ (s, $2 \mathrm{H}), 5.15(\mathrm{~d}, J=14.8 \mathrm{~Hz}, 1 \mathrm{H}), 4.96(\mathrm{~d}, J=16.0 \mathrm{~Hz}, 1 \mathrm{H}), 4.60$ (d, $J=$ 
$16.0 \mathrm{~Hz}, 1 \mathrm{H}), 4.45(\mathrm{~s}, 1 \mathrm{H}), 3.84(\mathrm{~d}, J=14.8 \mathrm{~Hz}, 1 \mathrm{H}), 3.79$ (s, $3 \mathrm{H}), 2.45-2.39$ (m, 1 H), 2.02 (s, $3 \mathrm{H}), 1.87-1.79(\mathrm{~m}, 1 \mathrm{H}), 1.62-1.55$ (m, $3 \mathrm{H}), 1.50-1.43$ (m, $1 \mathrm{H}), 1.32$ (d, $J=7.0 \mathrm{~Hz}, 3 \mathrm{H}), 1.30(\mathrm{~s}, 3 \mathrm{H}), 1.00(\mathrm{~s}, 3 \mathrm{H}), 0.85(\mathrm{~s}, 3 \mathrm{H}), 0.58-0.52(\mathrm{~m}, 2 \mathrm{H}), 0.44-$ $0.35(\mathrm{~m}, 1 \mathrm{H}) \mathrm{ppm} ;{ }^{13} \mathrm{C} \mathrm{NMR}\left(100 \mathrm{MHz}, \mathrm{CDCl}_{3}\right) \delta=205.8,169.8,167.5,159.4,150.69$, 139.8, 133.0, 129.9 (2C), 128.6, 121.9, 114.3 (2C), 73.7, 60.2, 59.9, 55.5, 48.0, 43.7, 42.7, 29.2, 28.6, 27.0, 22.2, 21.7, 20.4, 19.9, 18.3, 15.3, 14.5 ppm; HRMS (ESI): calcd for $\mathrm{C}_{30} \mathrm{H}_{39} \mathrm{NO}_{6} \mathrm{Na}^{+}[\mathrm{M}+\mathrm{Na}]^{+}$532.2675, found 532.2676.

(+)-Jatrophalactam (1): To a stirred solution of tricycle 25 (108 $\mathrm{mg}, 0.24 \mathrm{mmol})$ in a

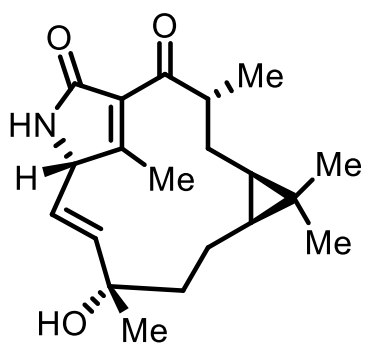

$(+)$-jatrophalactam (1) mixed solution of MeCN (10 mL) and phosphate buffer $(2 \mathrm{~mL}$, 2.0 $\mathrm{M}$ aq., $\mathrm{NaH}_{2} \mathrm{PO}_{4} / \mathrm{Na}_{2} \mathrm{HPO}_{4}$ 1:1) at room temperature was added CAN (658 mg, $1.2 \mathrm{mmol})$. The mixture was stirred for 2 h before it was diluted with $\mathrm{CH}_{2} \mathrm{Cl}_{2}(10 \mathrm{~mL})$ and $\mathrm{H}_{2} \mathrm{O}(10 \mathrm{~mL})$.

The layers were separated, and the aqueous layer was extracted with $\mathrm{CH}_{2} \mathrm{Cl}_{2}(3 \times 10$ $\mathrm{mL})$. The combined organic layers were dried $\left(\mathrm{Na}_{2} \mathrm{SO}_{4}\right)$ and concentrated in vacuo. Flash column chromatography (silica gel, EtOAc) afforded (+)-jatrophalactam (1, 60 mg, 75\%) as a white solid. $(+)-\mathbf{1}: R_{\mathrm{f}}=0.25$ (silica gel, EtOAc); m.p. $=184-185^{\circ} \mathrm{C}$ $\left(\right.$ EtOAc) $\left[\right.$ Lit. $185-186^{\circ} \mathrm{C}($ EtOAc $\left.)\right] ;^{6}[\alpha]_{\mathrm{D}}^{20}=+142.0(\mathrm{c}=0.3, \mathrm{MeOH})[$ Lit. $+148.7(\mathrm{c}$ $=0.3, \mathrm{MeOH})] ;{ }^{6} \mathrm{H} \mathrm{NMR}\left(400 \mathrm{MHz}, \mathrm{CDCl}_{3}\right) \delta=7.38(\mathrm{brs}, 1 \mathrm{H}), 5.82(\mathrm{~d}, J=16.0 \mathrm{~Hz}$, $1 \mathrm{H}), 5.74(\mathrm{dd}, J=16.0,3.5 \mathrm{~Hz}, 1 \mathrm{H}), 4.57$ (d, $J=3.5 \mathrm{~Hz}, 1 \mathrm{H}), 2.88$ (brs, $1 \mathrm{H}), 2.49$ $2.40(\mathrm{~m}, 1 \mathrm{H}), 2.01(\mathrm{~s}, 3 \mathrm{H}), 1.84-1.76(\mathrm{~m}, 1 \mathrm{H}), 1.59-1.50(\mathrm{~m}, 1 \mathrm{H}), 1.45-1.41(\mathrm{~m}, 1$ H), 1.35-1.33 (m, $1 \mathrm{H}), 1.32-1.28(\mathrm{~m}, 1 \mathrm{H}), 1.27(\mathrm{~s}, 3 \mathrm{H}), 1.25(\mathrm{~d}, J=6.8 \mathrm{~Hz}, 3 \mathrm{H})$, 0.98 (s, $3 \mathrm{H}), 0.89$ (s, $3 \mathrm{H}), 0.55-0.51(\mathrm{~m}, 1 \mathrm{H}), 0.53-0.48(\mathrm{~m}, 1 \mathrm{H}), 0.45-0.36 \mathrm{ppm}(\mathrm{m}$, 
$1 \mathrm{H}) ;{ }^{13} \mathrm{C} \mathrm{NMR}\left(100 \mathrm{MHz}, \mathrm{CDCl}_{3}\right) \delta=205.8,171.8,157.2,138.0,131.1,123.9,73.7$, $60.3,47.8,42.2,29.3,28.4,26.9,22.2,21.8,19.9,18.4,15.6,14.7,13.7$ ppm; HRMS (ESI): calcd for $\mathrm{C}_{20} \mathrm{H}_{29} \mathrm{NO}_{3} \mathrm{Na}^{+}[\mathrm{M}+\mathrm{Na}]^{+}$354.2045, found 354.2043. 
Table S1. ${ }^{1} \mathrm{H}$ NMR Spectroscopic $\left(\mathrm{CDCl}_{3}\right)$ Comparison of Natural ${ }^{6}$ and Synthetic (+)Jatrophalactam (1).

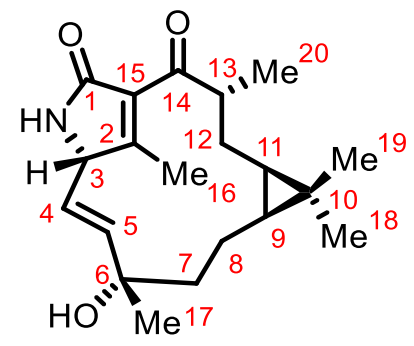

(+)-jatrophalactam (1)

\begin{tabular}{ccc}
\hline No. & Natural & Synthetic \\
& $\delta{ }^{1} \mathrm{H}[\mathrm{ppm}$, mult, $J(\mathrm{~Hz})]$ & $\delta{ }^{1} \mathrm{H}[\mathrm{ppm}$, mult, $J(\mathrm{~Hz})]$ \\
\hline 1 & & \\
2 & & $4.57(\mathrm{~d}, J=3.5 \mathrm{~Hz})$ \\
3 & $4.56(\mathrm{~d}, J=3.3 \mathrm{~Hz})$ & $5.74(\mathrm{dd}, J=16.0,3.5 \mathrm{~Hz})$ \\
4 & $5.75(\mathrm{dd}, J=3.3,16.2 \mathrm{~Hz})$ & $5.82(\mathrm{~d}, J=16.0 \mathrm{~Hz})$ \\
5 & $5.84(\mathrm{~d}, J=16.2 \mathrm{~Hz})$ & \\
6 & & $1.35-1.33(\mathrm{~m}), 1.84-1.76(\mathrm{~m})$ \\
7 & $1.33(\mathrm{~m}), 1.77(\mathrm{~m})$ & $0.55-0.51(\mathrm{~m})$ \\
8 & $1.58(\mathrm{~m})$ & $0.53-0.48(\mathrm{~m})$ \\
9 & $0.53(\mathrm{~m})$ & $1.32-1.28(\mathrm{~m}), 1.45-1.41(\mathrm{~m})$ \\
10 & & $2.49-2.40(\mathrm{~m})$ \\
11 & $0.43(\mathrm{~m})$ & \\
12 & $1.42(\mathrm{~m}), 1.48(\mathrm{~m})$ & \\
13 & $2.45(\mathrm{~m})$ & $2.01(\mathrm{~s})$ \\
14 & & $1.27(\mathrm{~s})$ \\
15 & & $0.98(\mathrm{~s})$ \\
16 & $2.01(\mathrm{~s})$ & $0.89(\mathrm{~s})$ \\
17 & $1.28(\mathrm{~s})$ & $1.25(\mathrm{~d}, J=6.9 \mathrm{~Hz})$ \\
18 & $0.98(\mathrm{~s})$ & $2.88(\mathrm{brs})$ \\
19 & $0.89(\mathrm{~s})$ & \\
20 & $1.26(\mathrm{~d}, J=7.2 \mathrm{~Hz})$ & \\
$\mathrm{NH}$ & $7.00(\mathrm{brs})$ & \\
$\mathrm{OH}$ & & \\
\hline
\end{tabular}


Table S2. ${ }^{13} \mathrm{C}$ NMR $\left(\mathrm{CDCl}_{3}\right)$ Spectroscopic Comparison of Natural ${ }^{6}$ and Synthetic (+)Jatrophalactam (1).

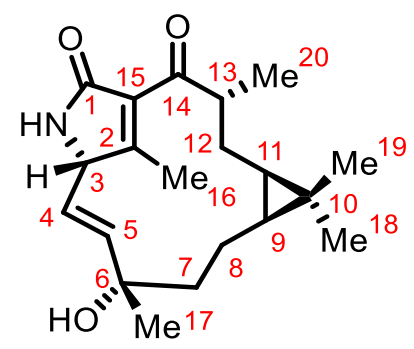

$(+)$-jatrophalactam (1)

\begin{tabular}{ccc}
\hline No. & $\begin{array}{c}\text { Natural } \\
\delta{ }^{13} \mathrm{C}(\mathrm{ppm})\end{array}$ & $\begin{array}{c}\text { Synthetic } \\
{ }^{13} \mathrm{C}(\mathrm{ppm})\end{array}$ \\
\hline 1 & 171.9 & 171.8 \\
2 & 131.0 & 131.1 \\
3 & 60.3 & 60.3 \\
4 & 123.8 & 123.9 \\
5 & 138.1 & 138.0 \\
6 & 73.7 & 73.7 \\
7 & 42.2 & 42.2 \\
8 & 20.0 & 19.9 \\
9 & 27.0 & 26.9 \\
10 & 18.4 & 18.4 \\
11 & 21.8 & 21.8 \\
12 & 28.4 & 28.4 \\
13 & 47.7 & 47.8 \\
14 & 205.8 & 205.8 \\
15 & 157.4 & 157.2 \\
16 & 14.7 & 14.7 \\
17 & 22.1 & 22.2 \\
18 & 29.4 & 29.3 \\
19 & 15.6 & 15.6 \\
20 & 13.7 & 13.7 \\
\hline
\end{tabular}




\section{II) Abbreviations}

CAN ceric ammonium nitrate

DEAD diethyl azodicarboxylate

DMAP $\quad N, N^{\prime}$-dimethylaminopyridine

DME 1,2-dimethoxyethane

EDCI 1-(3-dimethylaminopropyl)-3-ethylcarbodiimide hydrochloride

HMPA hexamethylphosphoric triamide

LDA lithium diisopropylamide

LiHMDS lithium bis(trimethylsilyl)amide

Meldrum's acid 2,2-dimethyl-1,3-dioxane-4,6-dione

PPTS pyridinium 4-toluenesulfonate

PMP 4-methoxybenzyl

PMTA 1-phenyl-1 $H$-tetrazole-5-thiol

TBSOTf tert-butyldimethylsilyl trifluoromethanesulfonate

TEMPO 2,2,6,6-tetramethylpiperidine-1-oxyl

TFA trifluoroacetic acid

THF tetrahydrofuran

$\mathrm{TsCl} \quad$ 4-toluene sulfonyl chloride

$4 \AA \mathrm{MS} \quad 4 \AA$ molecular sieves 


\section{III) References}

(1) Xu, D.; Crispino, G. A.; Sharpless, K. B. J. Am. Chem. Soc. 1992, 114, 7570.

(2) Riley, R. G.; Silverstein, R. M. Tetrahedron 1974, 30, 1171.

(3) Kutsumura, N.; Nishiyama, S. J. Carbohyd. Chem. 2006, 25, 377.

(4) Moriwake, T.; Hamano, S.; Saito, S.; Torii, S. Chem. Lett. 1987, 2085.

(5) Agawa, T.; Kubo, T.; Ohshiro, Y. Synthesis 1971, 27.

(6) Wang, X.-C.; Zheng, Z.-P.; Gan, X.-W.; Hu, L.-H. Org. Lett. 2009, 11, 5522. 
IV) ${ }^{1} \mathrm{H}$ and ${ }^{13} \mathrm{C}$ NMR Spectra of Compounds

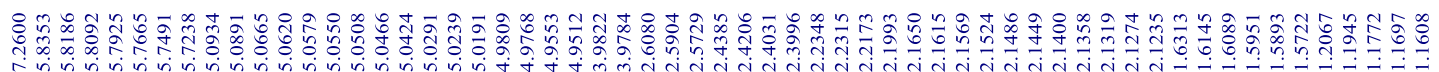<smiles>C=CCC[C@](C)(O)COC(=O)[C@H](C)CC=C</smiles>

11a

${ }^{1} \mathrm{H}$ NMR spectrum $\left(400 \mathrm{MHz}, \mathrm{CDCl}_{3}\right)$

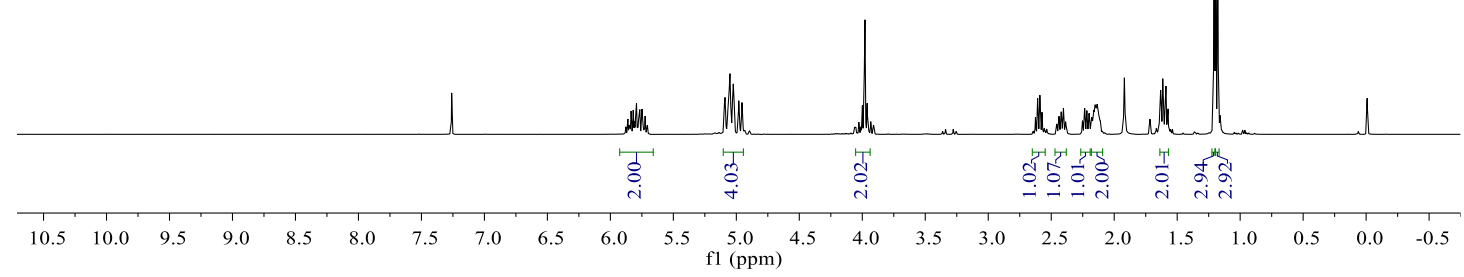

\begin{tabular}{|c|c|c|c|c|}
\hline $\begin{array}{l}\frac{n}{6} \\
\frac{1}{1} \\
1\end{array}$ & $\begin{array}{ll}n & 0 \\
n & n \\
\infty & n \\
m & n \\
1 & 1\end{array}$ & 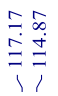 & 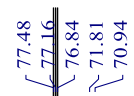 & 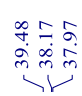 \\
\hline
\end{tabular}

${ }^{13} \mathrm{C}$ NMR spectrum $\left(100 \mathrm{MHz}, \mathrm{CDCl}_{3}\right)$

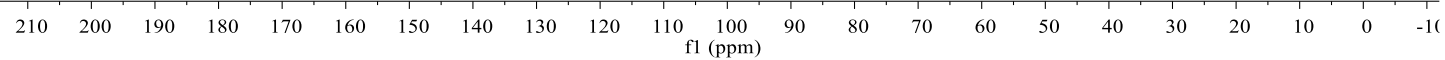




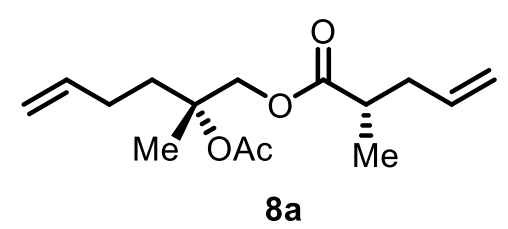

${ }^{1} \mathrm{H}$ NMR spectrum (400 MHz, $\mathrm{CDCl}_{3}$ )

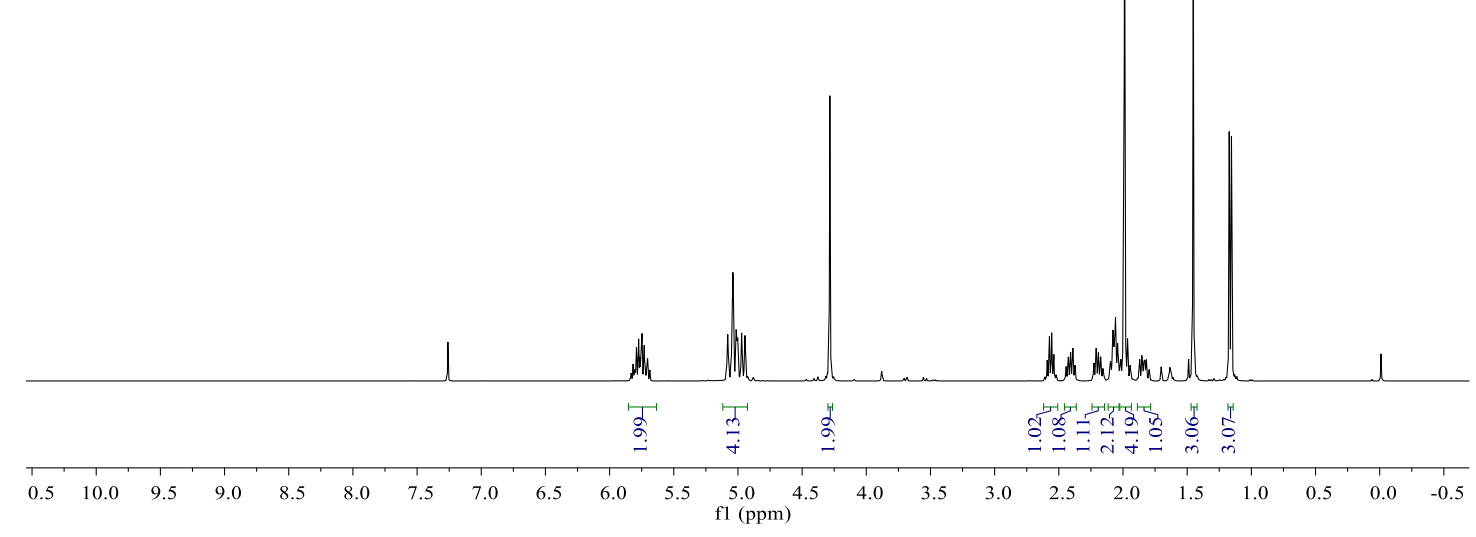
量

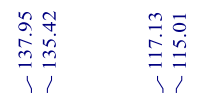

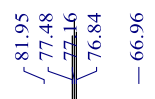

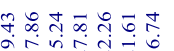
imminat

${ }^{13} \mathrm{C}$ NMR spectrum $\left(100 \mathrm{MHz}, \mathrm{CDCl}_{3}\right)$

$\begin{array}{llllllllllllllllllllllll}210 & 200 & 190 & 180 & 170 & 160 & 150 & 140 & 130 & 120 & 110 & 100 & 90 & 80 & 70 & 60 & 50 & 40 & 30 & 20 & 10 & 0 & -1(\end{array}$ 
<smiles></smiles>

${ }^{1} \mathrm{H}$ NMR spectrum $\left(400 \mathrm{MHz}, \mathrm{CDCl}_{3}\right)$

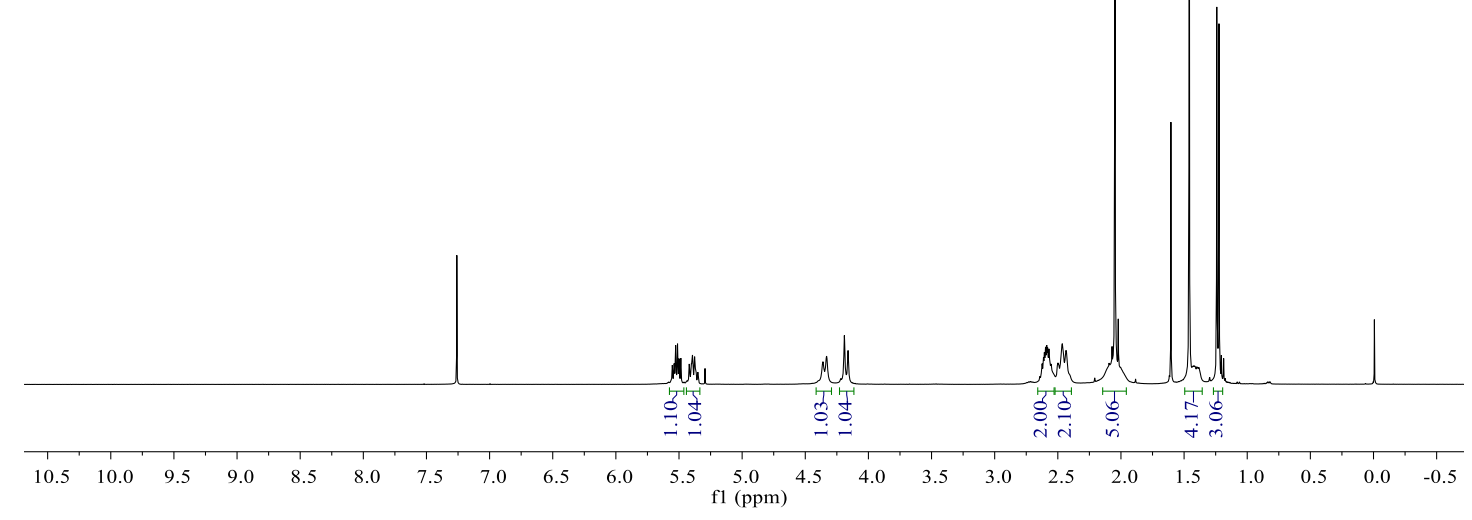

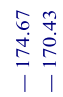

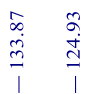

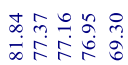
Б용요
然
अल तुत

${ }^{13} \mathrm{C}$ NMR spectrum (150 MHz, $\left.\mathrm{CDCl}_{3}\right)$

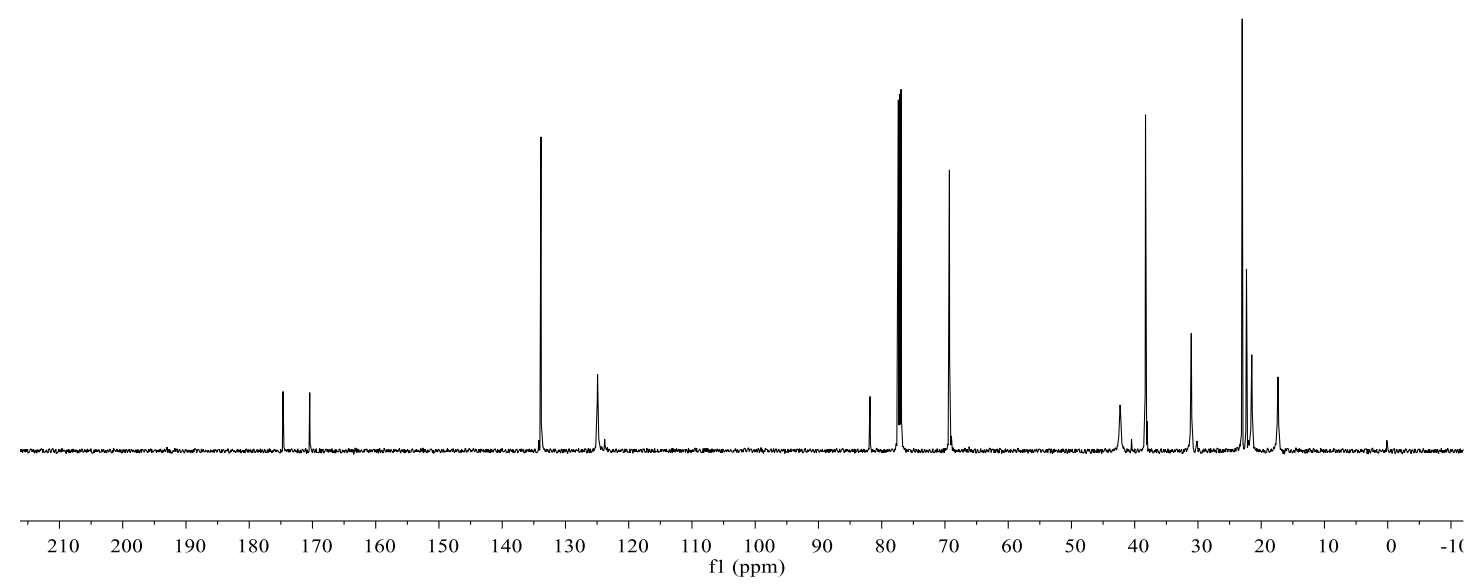




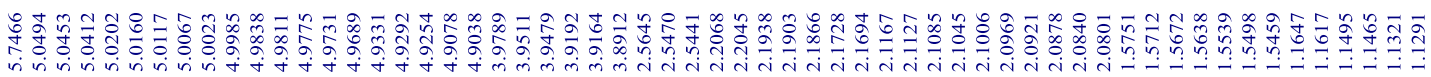<smiles>C=CCC[C@](C)(O)COC(=O)[C@H](C)CC=C</smiles>

$11 b$

${ }^{1} \mathrm{H}$ NMR spectrum $\left(400 \mathrm{MHz}, \mathrm{CDCl}_{3}\right)$

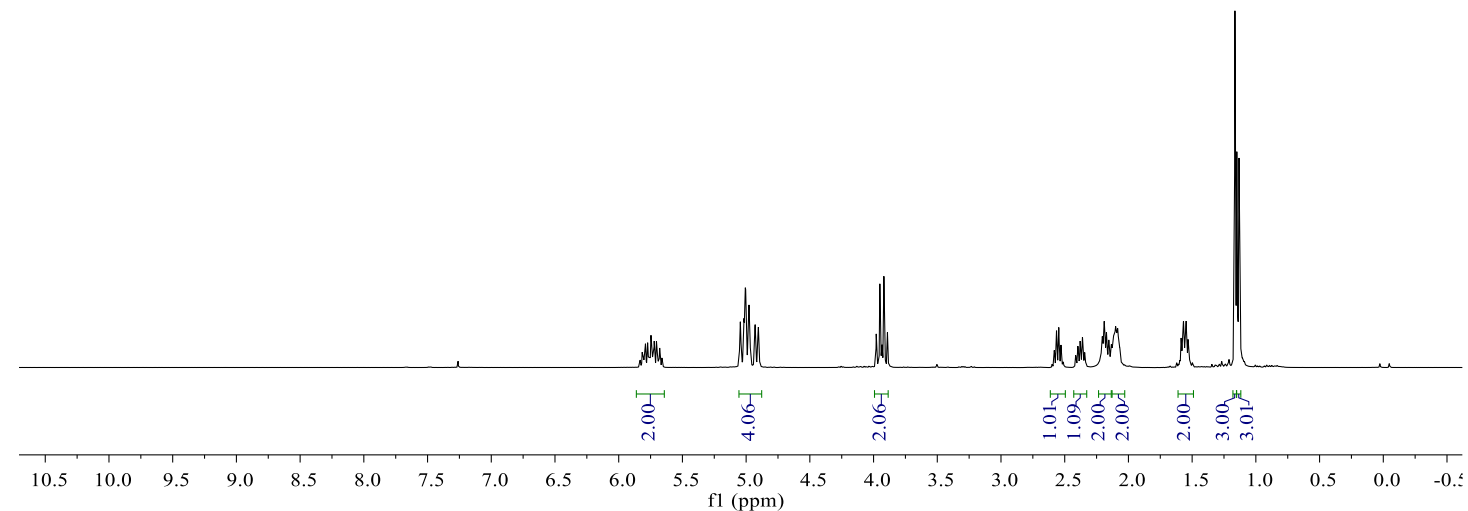

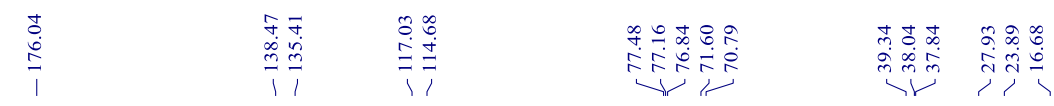

${ }^{13} \mathrm{C}$ NMR spectrum $\left(100 \mathrm{MHz}, \mathrm{CDCl}_{3}\right)$

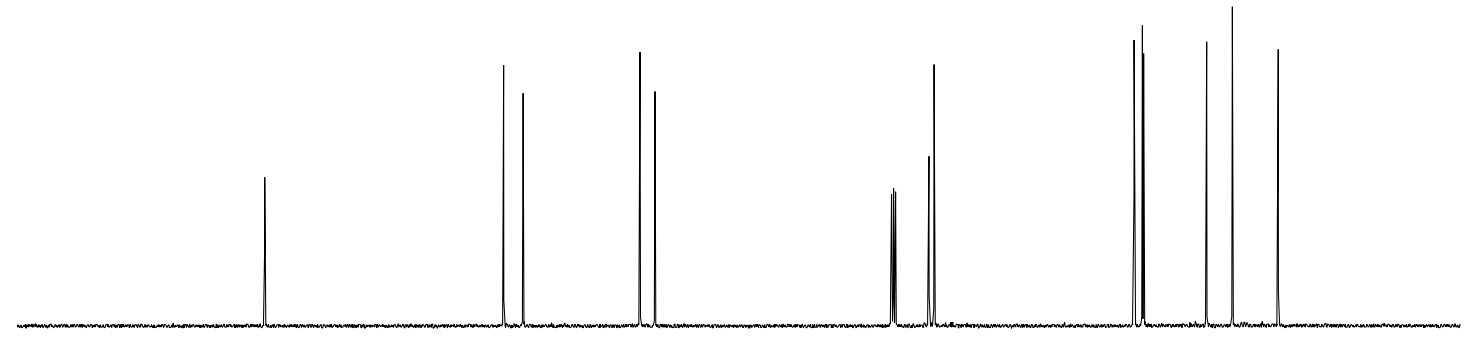

$\begin{array}{lllllllllllllllllllllll}210 & 200 & 190 & 180 & 170 & 160 & 150 & 140 & 130 & 120 & 110 & 100 & 90 & 80 & 70 & 60 & 50 & 40 & 30 & 20 & 10 & 0 & -1(\end{array}$ 


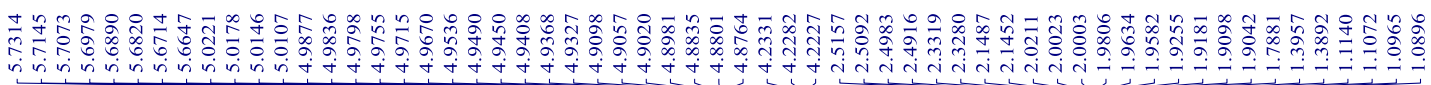<smiles>C=CCC[C@@](C)(COC(=O)[C@@H](C)CC=C)OC(C)=O</smiles>

$8 b$

${ }^{1} \mathrm{H}$ NMR spectrum $\left(400 \mathrm{MHz}, \mathrm{CDCl}_{3}\right)$

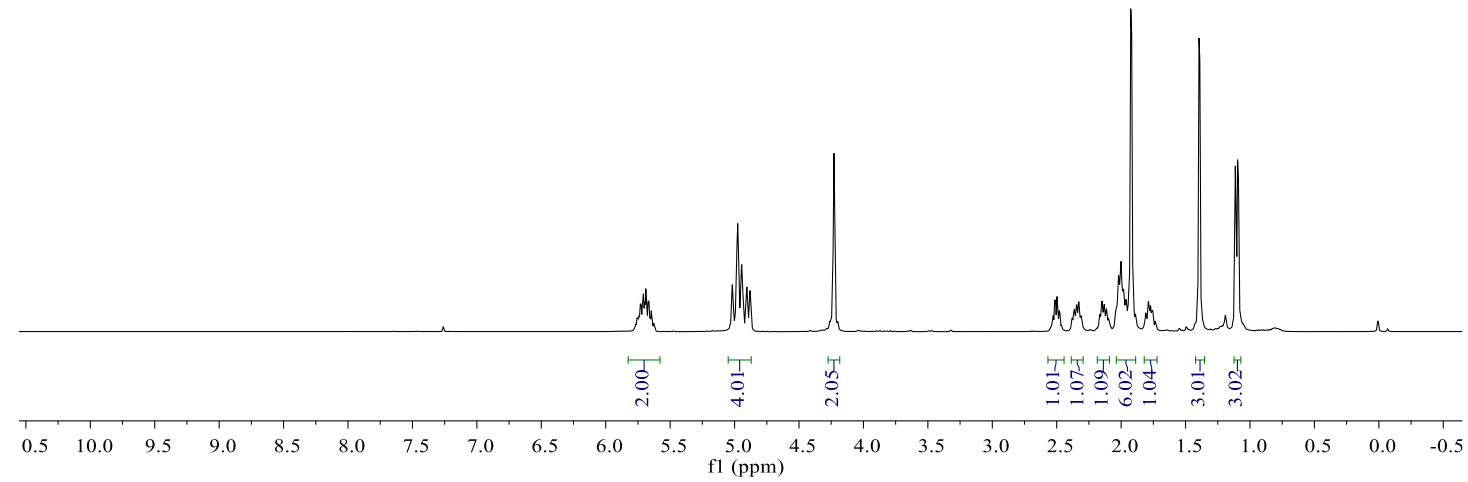

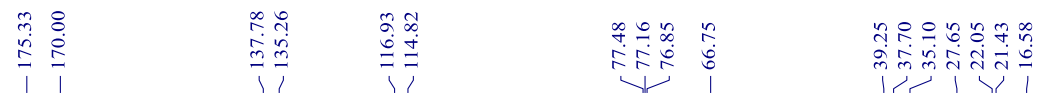

${ }^{13} \mathrm{C}$ NMR spectrum $\left(100 \mathrm{MHz}, \mathrm{CDCl}_{3}\right)$

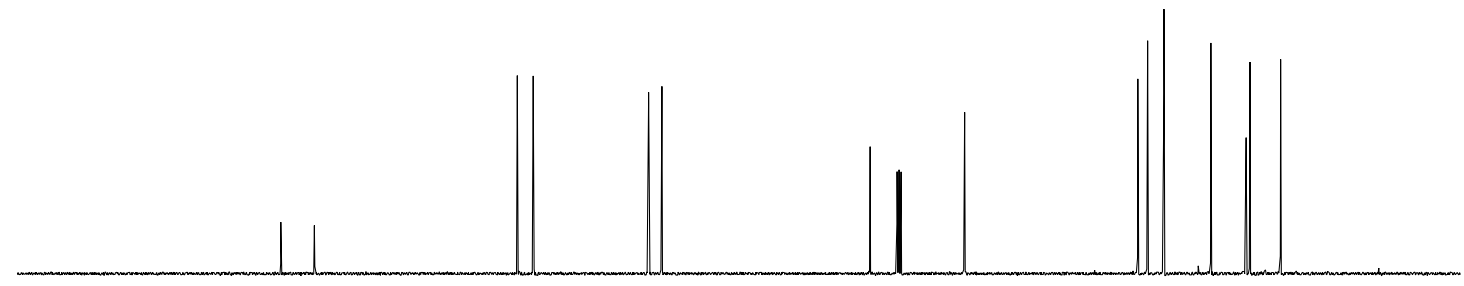

$\begin{array}{lllllllllllllllllllllll}210 & 200 & 190 & 180 & 170 & 160 & 150 & 140 & 130 & 120 & 110 & 100 & 90 & 80 & 70 & 60 & 50 & 40 & 30 & 20 & 10 & 0 & -1(1\end{array}$ 
<smiles></smiles>

${ }^{1} \mathrm{H}$ NMR spectrum $\left(400 \mathrm{MHz}, \mathrm{CDCl}_{3}\right)$

\section{wh}

M.
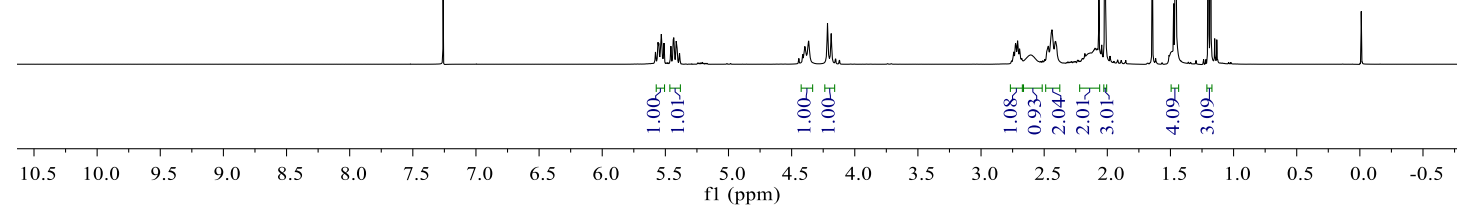

$$
\frac{1}{\text { ì }}
$$

${ }^{13} \mathrm{C}$ NMR spectrum $\left(100 \mathrm{MHz}, \mathrm{CDCl}_{3}\right)$
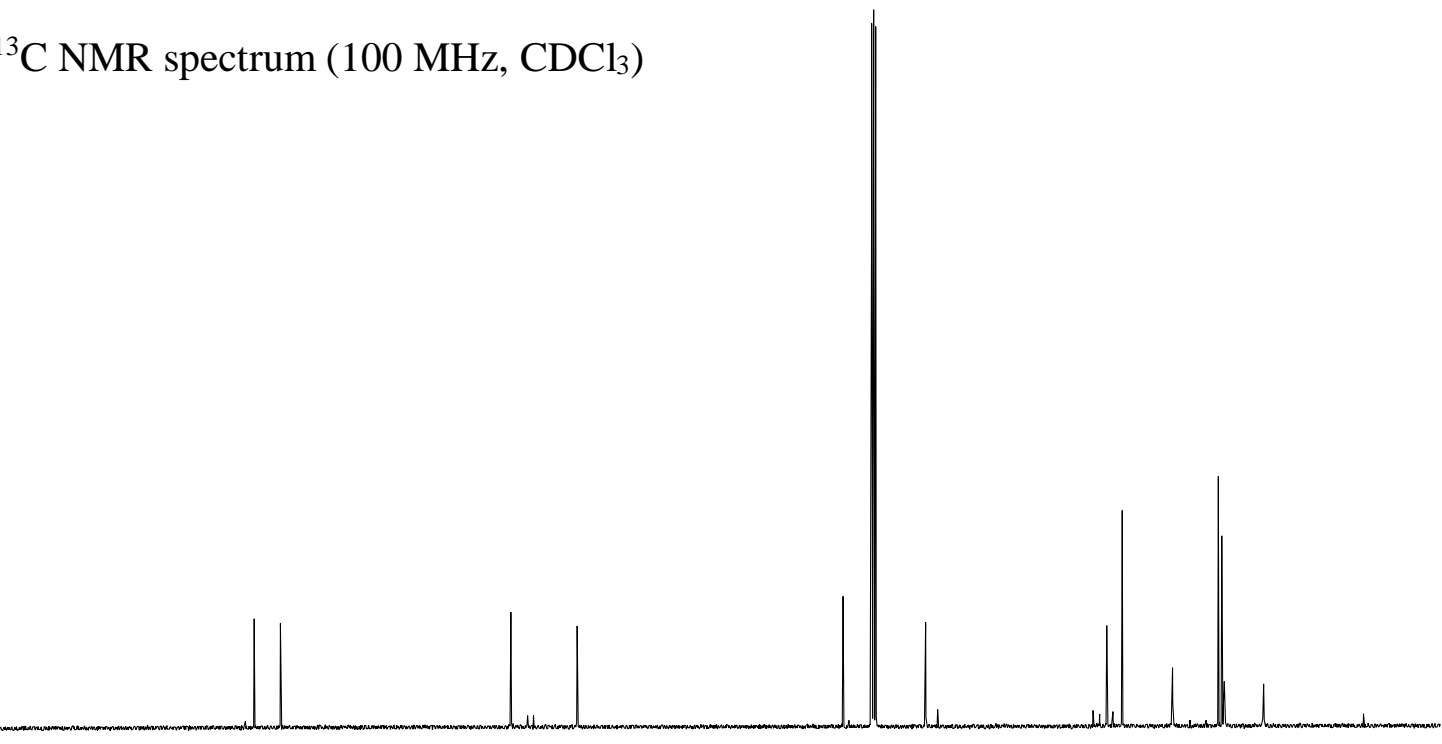

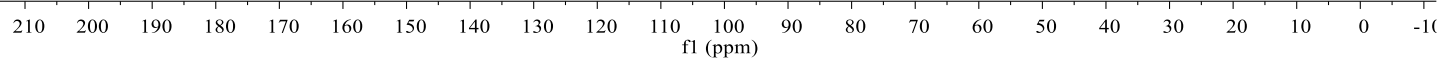



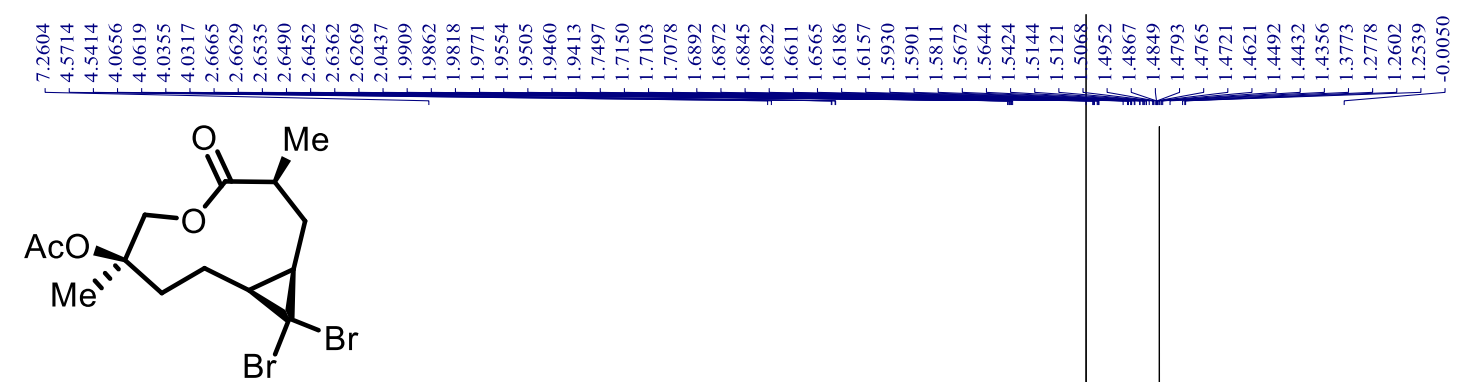

12

${ }^{1} \mathrm{H}$ NMR spectrum $\left(400 \mathrm{MHz}, \mathrm{CDCl}_{3}\right)$

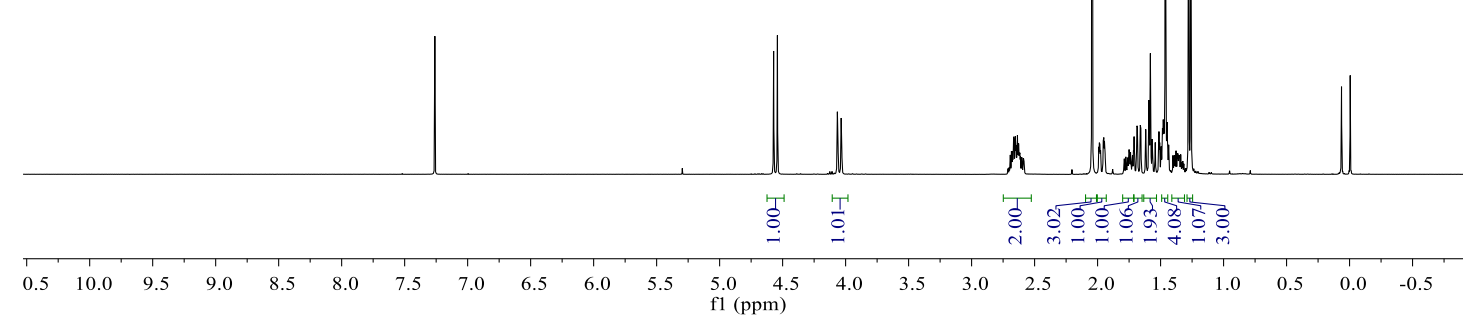

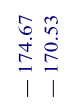

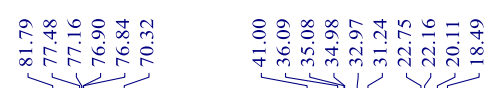

नल mm लm

${ }^{13} \mathrm{C}$ NMR spectrum $\left(100 \mathrm{MHz}, \mathrm{CDCl}_{3}\right)$

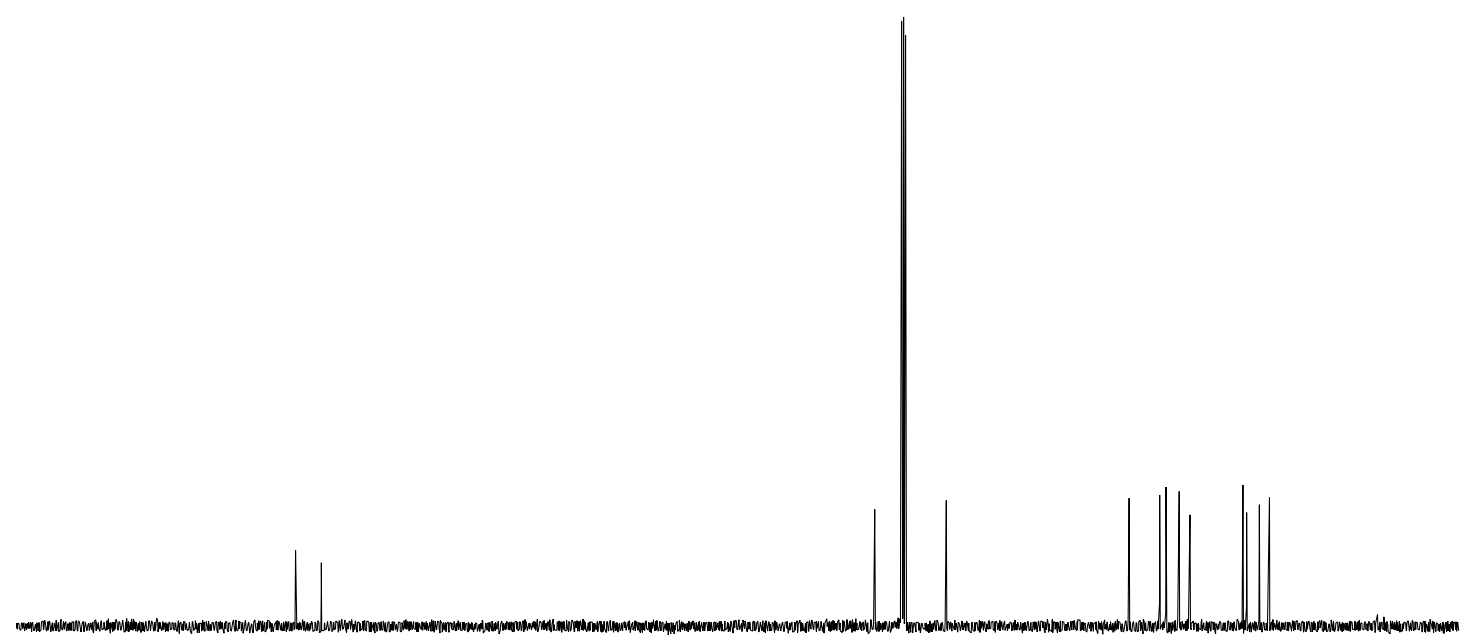

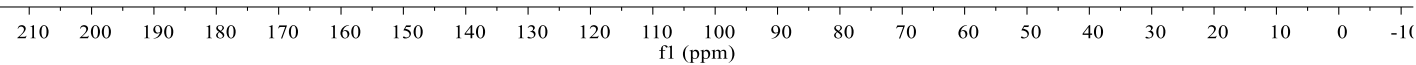



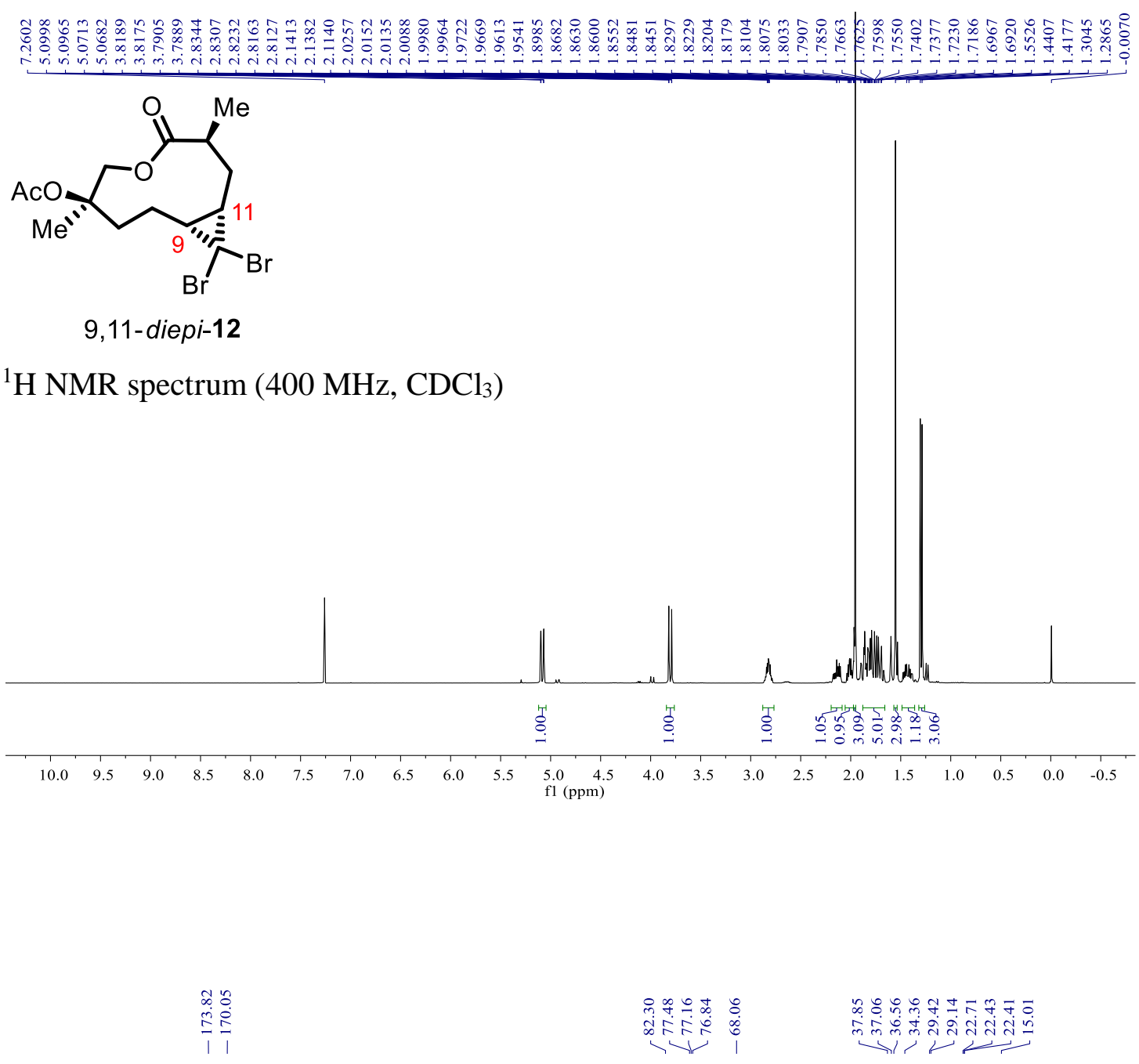

${ }^{13} \mathrm{C}$ NMR spectrum $\left(100 \mathrm{MHz}, \mathrm{CDCl}_{3}\right)$

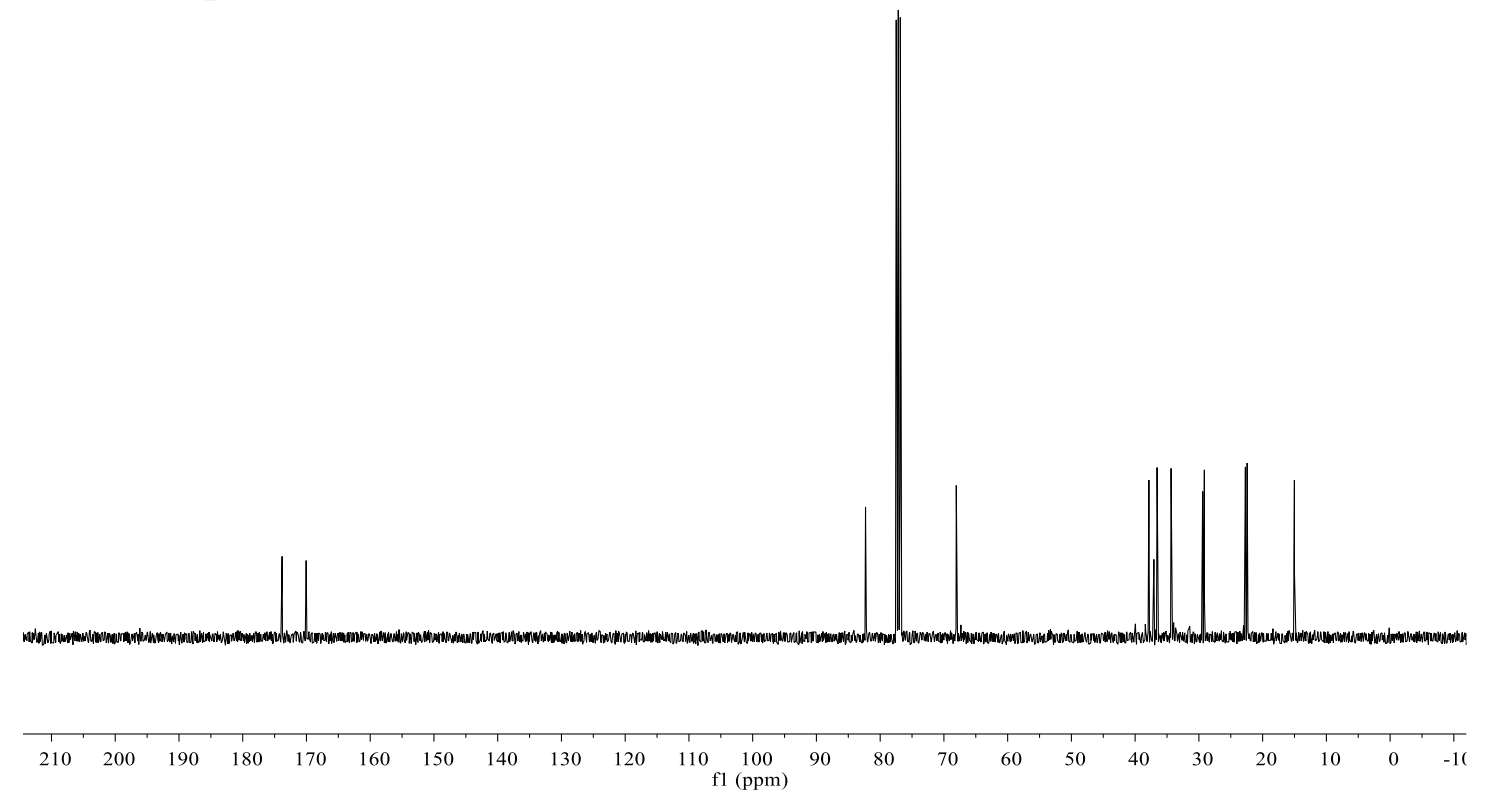


<smiles>[Y4]C1CC2C(CC[C@](C)(OC(C)C)C2(Br)Br)COC1=O</smiles>

6-epi-12

${ }^{1} \mathrm{H}$ NMR spectrum $\left(400 \mathrm{MHz}, \mathrm{CDCl}_{3}\right)$

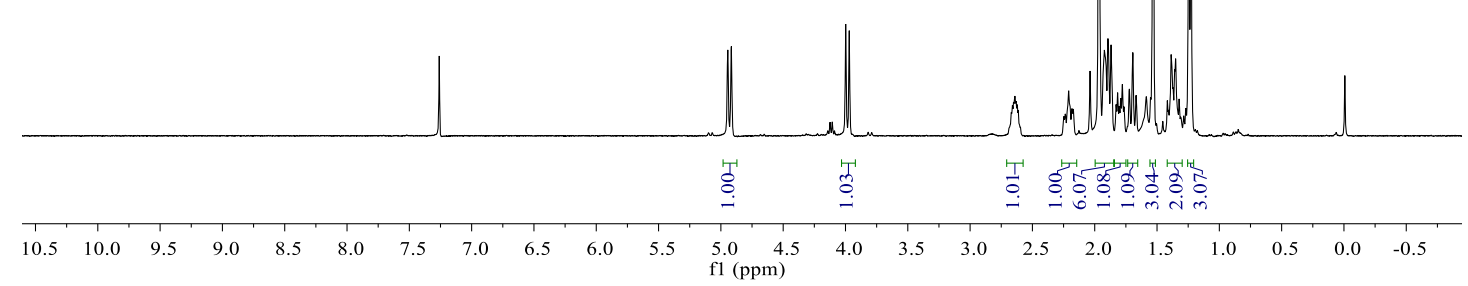

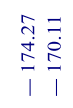

${ }^{13} \mathrm{C}$ NMR spectrum $\left(100 \mathrm{MHz}, \mathrm{CDCl}_{3}\right)$

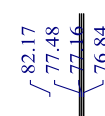




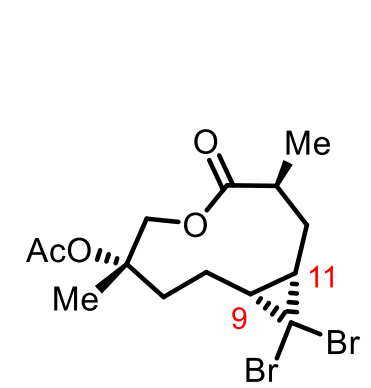

6,9,11-triepi-12

${ }^{1} \mathrm{H}$ NMR spectrum $\left(400 \mathrm{MHz}, \mathrm{CDCl}_{3}\right)$

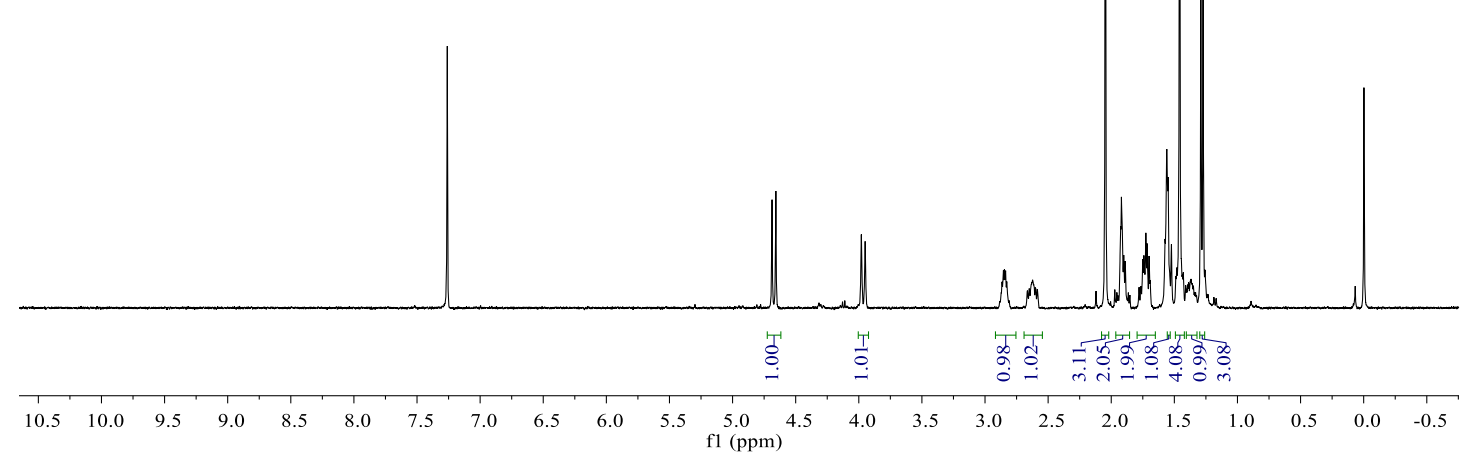

${ }^{13} \mathrm{C}$ NMR spectrum $\left(100 \mathrm{MHz}, \mathrm{CDCl}_{3}\right)$

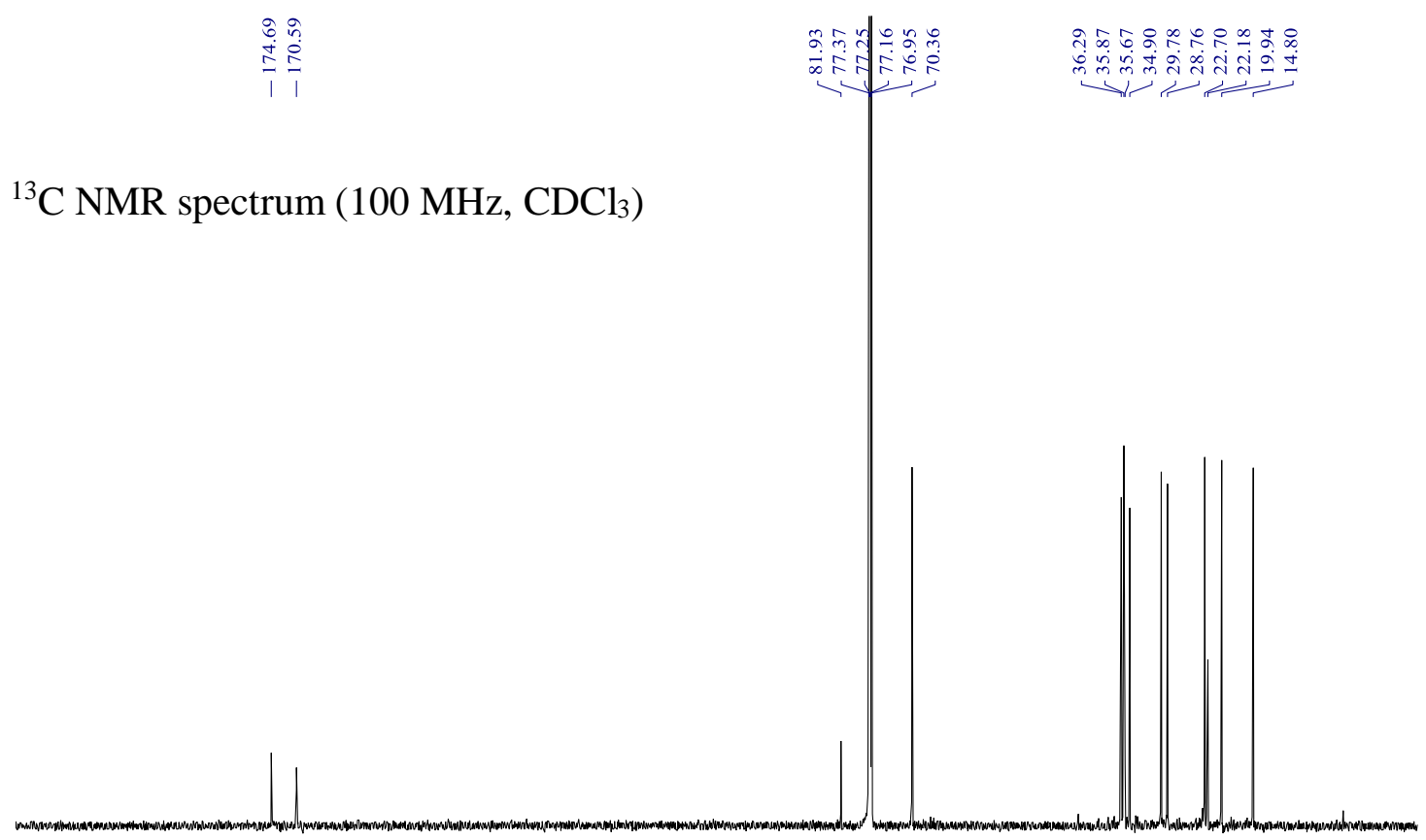

$\begin{array}{lllllllllllllllllllllll}210 & 200 & 190 & 180 & 170 & 160 & 150 & 140 & 130 & 120 & 110 & 100 & 90 & 80 & 70 & 60 & 50 & 40 & 30 & 20 & 10 & 0 & -1(\end{array}$ 

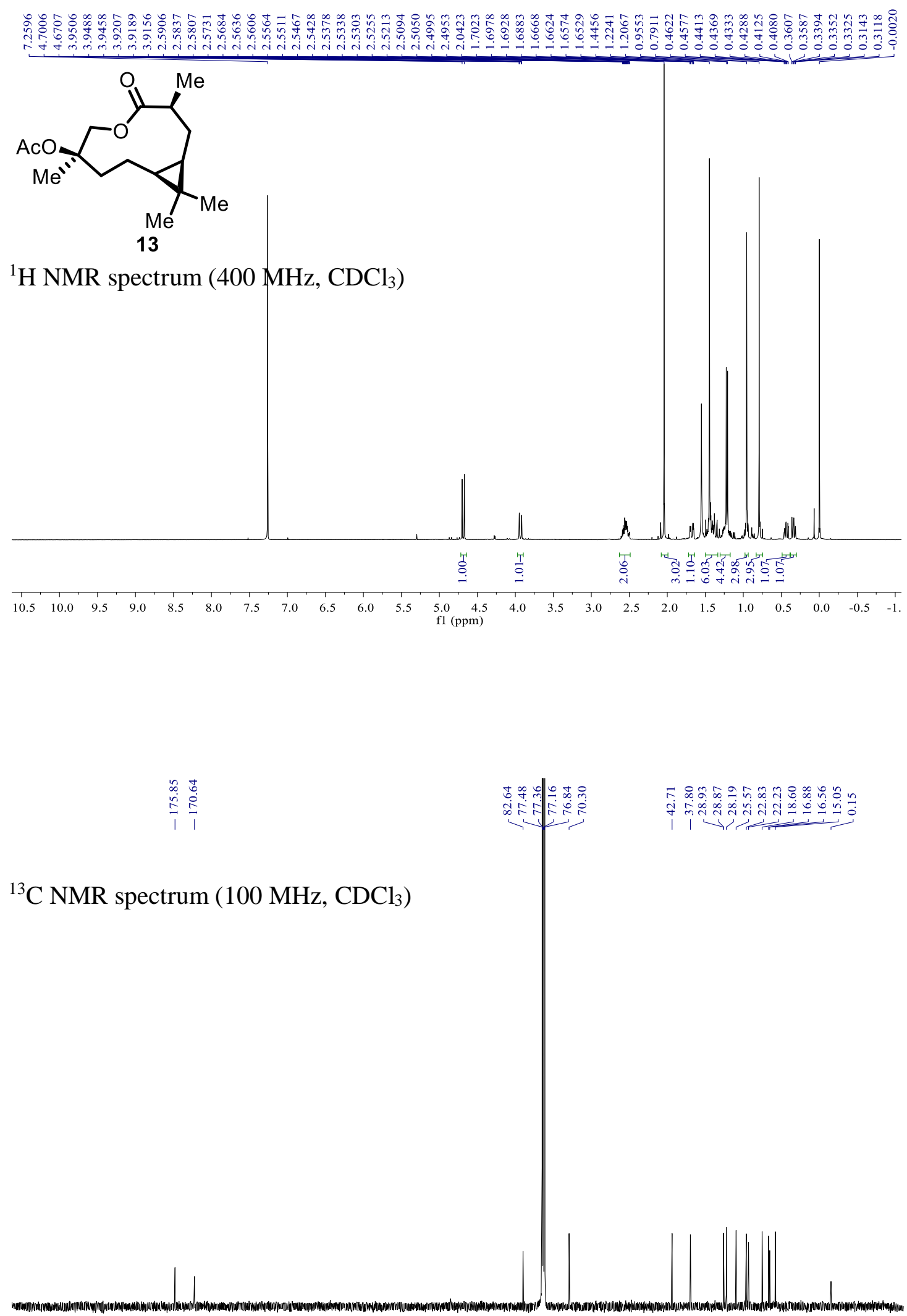

$\begin{array}{llllllllllllllllllllllll}1 & 210 & 200 & 190 & 180 & 170 & 160 & 150 & 140 & 130 & 120 & 110 & 100 & 90 & 80 & 70 & 60 & 50 & 40 & 30 & 20 & 10 & 0 & -10\end{array}$ 

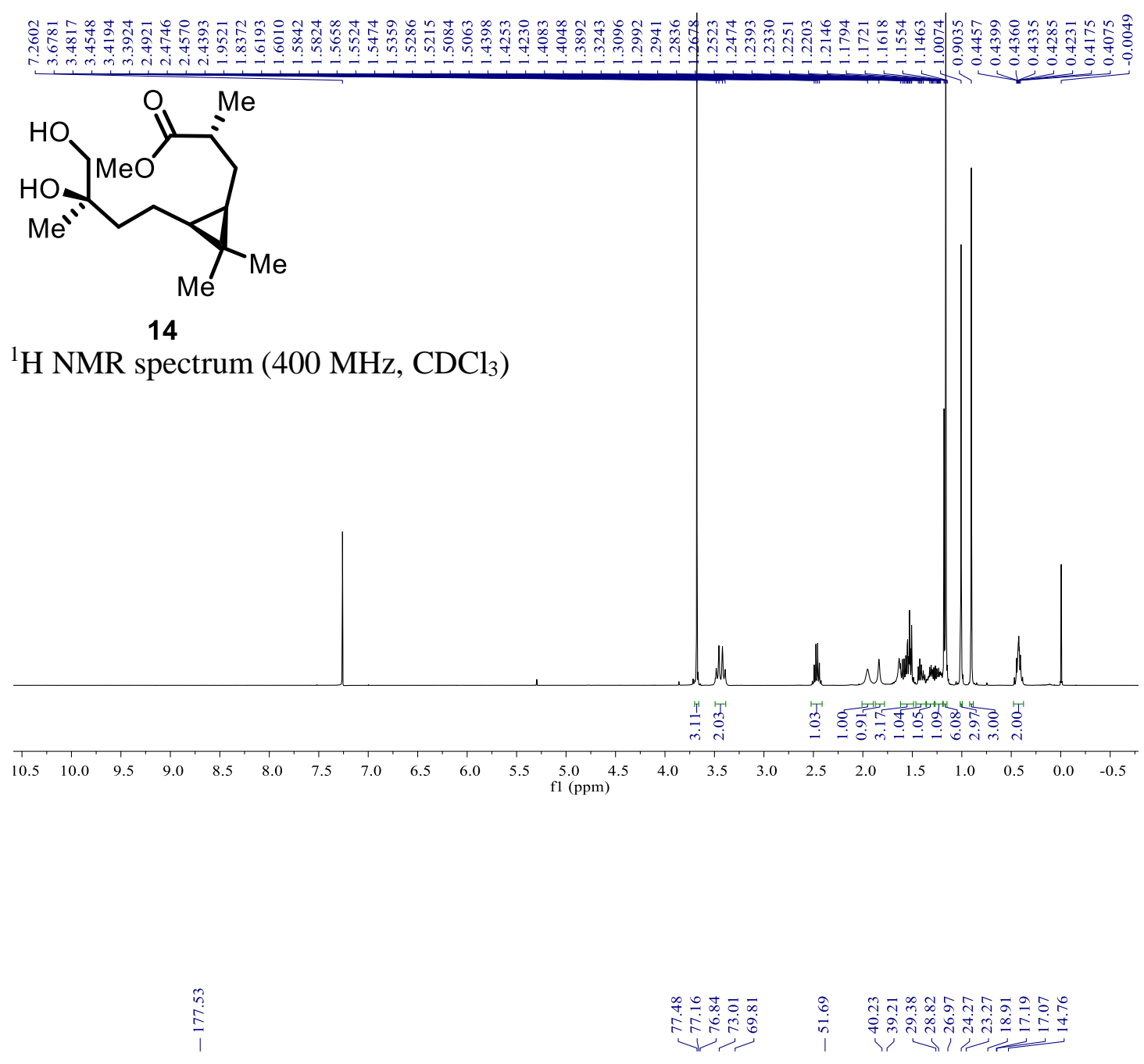

${ }^{13} \mathrm{C}$ NMR spectrum $\left(100 \mathrm{MHz}, \mathrm{CDCl}_{3}\right)$

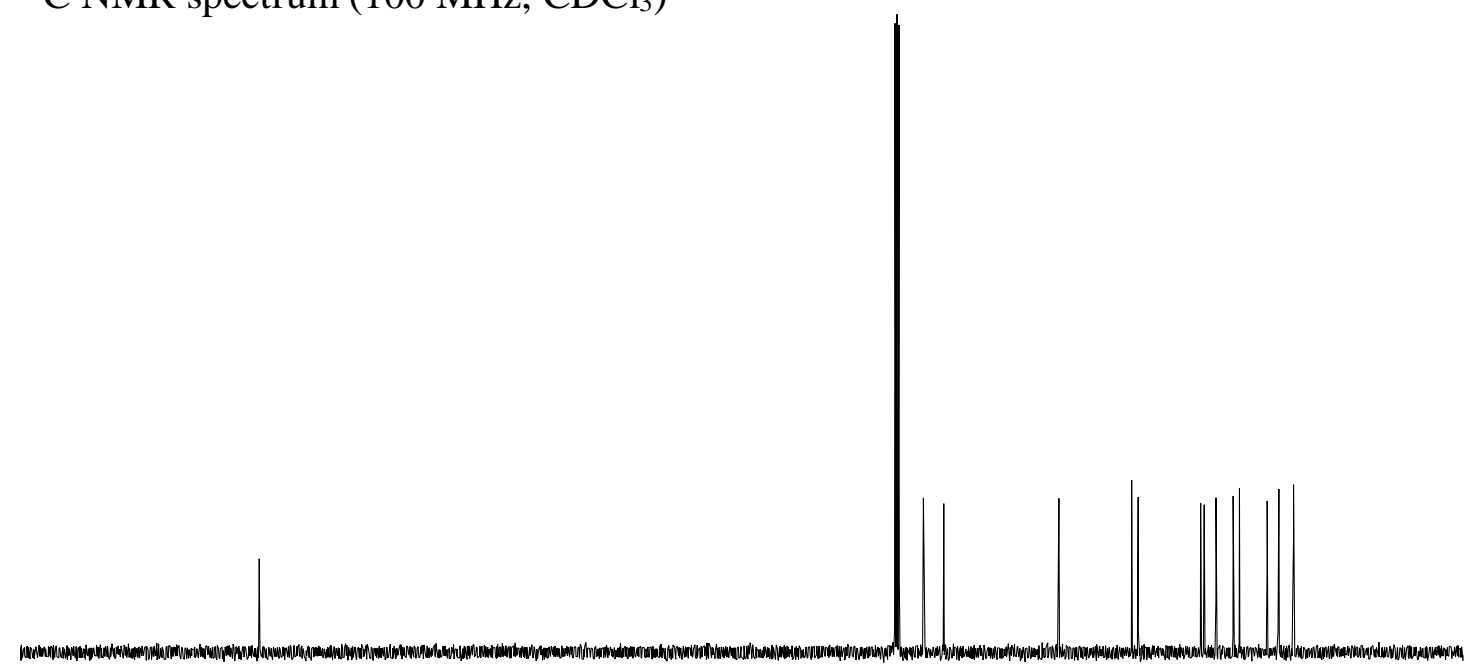

$\begin{array}{lllllllllllllllllllllll}210 & 200 & 190 & 180 & 170 & 160 & 150 & 140 & 130 & 120 & 110 & 100 & 90 & 80 & 70 & 60 & 50 & 40 & 30 & 20 & 10 & 0 & -11\end{array}$ 

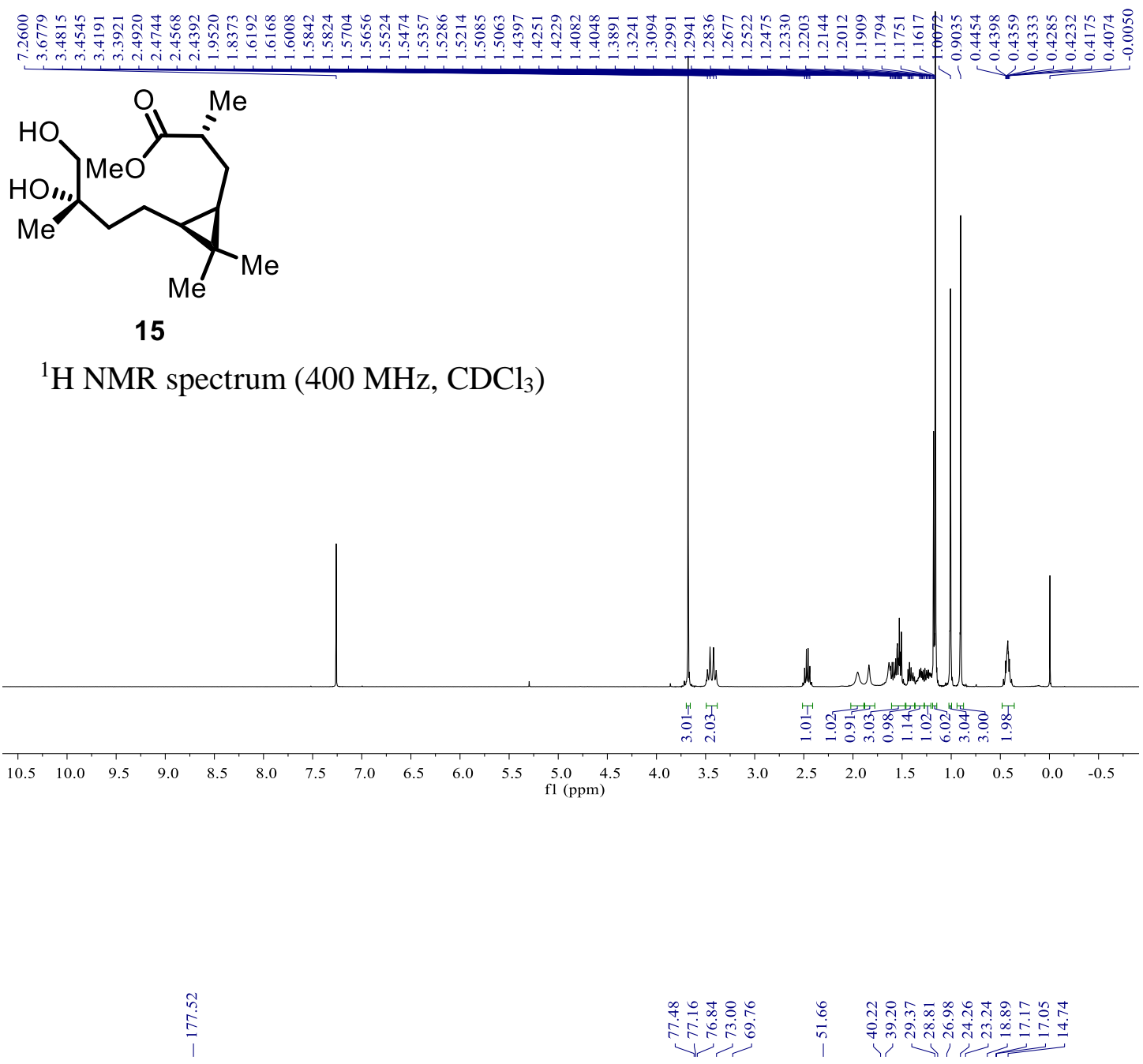

${ }^{13} \mathrm{C}$ NMR spectrum $\left(100 \mathrm{MHz}, \mathrm{CDCl}_{3}\right)$

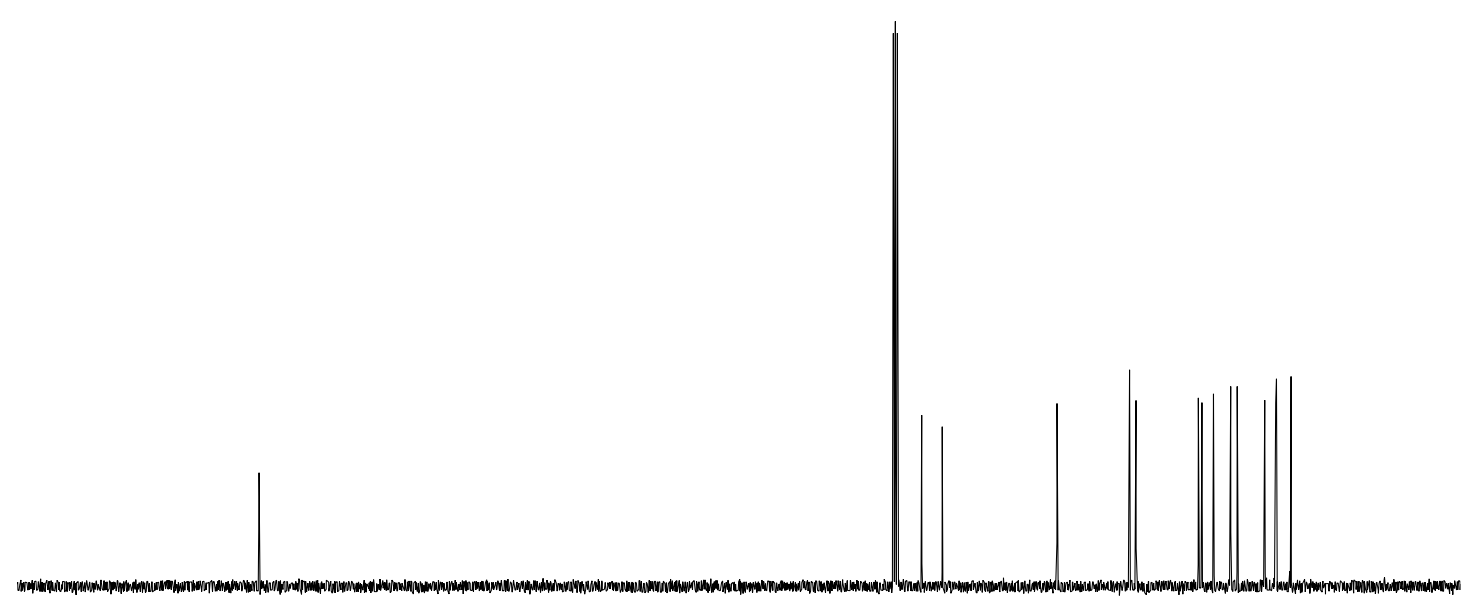

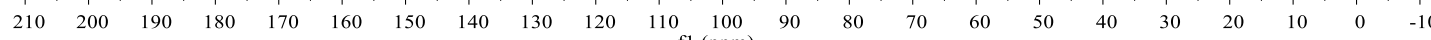




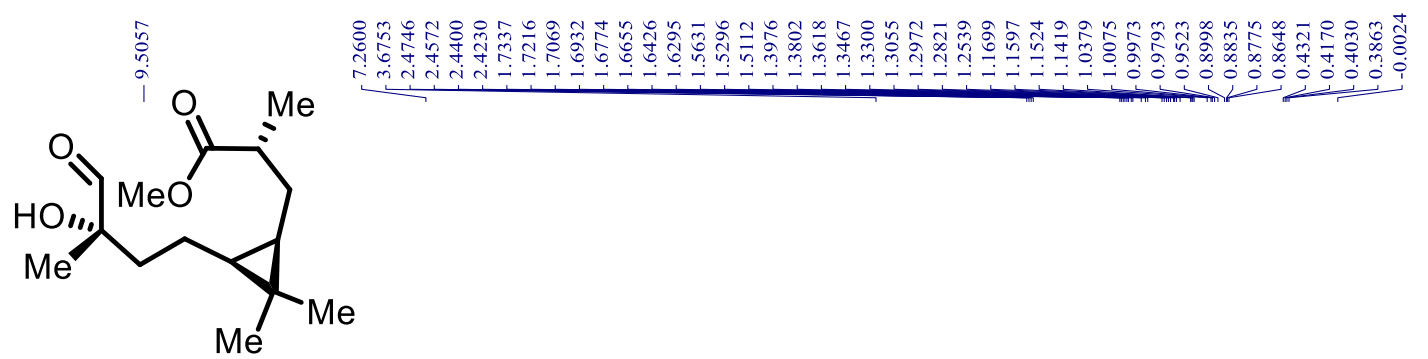

16

${ }^{1} \mathrm{H}$ NMR spectrum (400 MHz, $\mathrm{CDCl}_{3}$ )

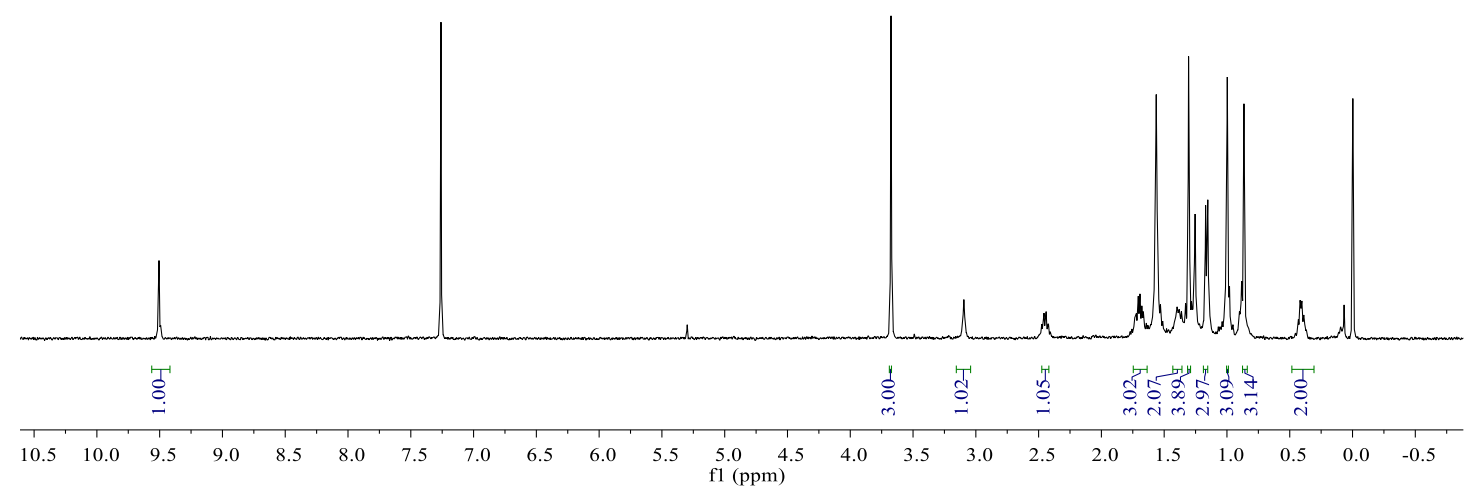

$\begin{array}{ll}\infty & \vec{n} \\ \infty & \stackrel{5}{\infty}\end{array}$

${ }^{13} \mathrm{C}$ NMR spectrum $\left(100 \mathrm{MHz}, \mathrm{CDCl}_{3}\right)$
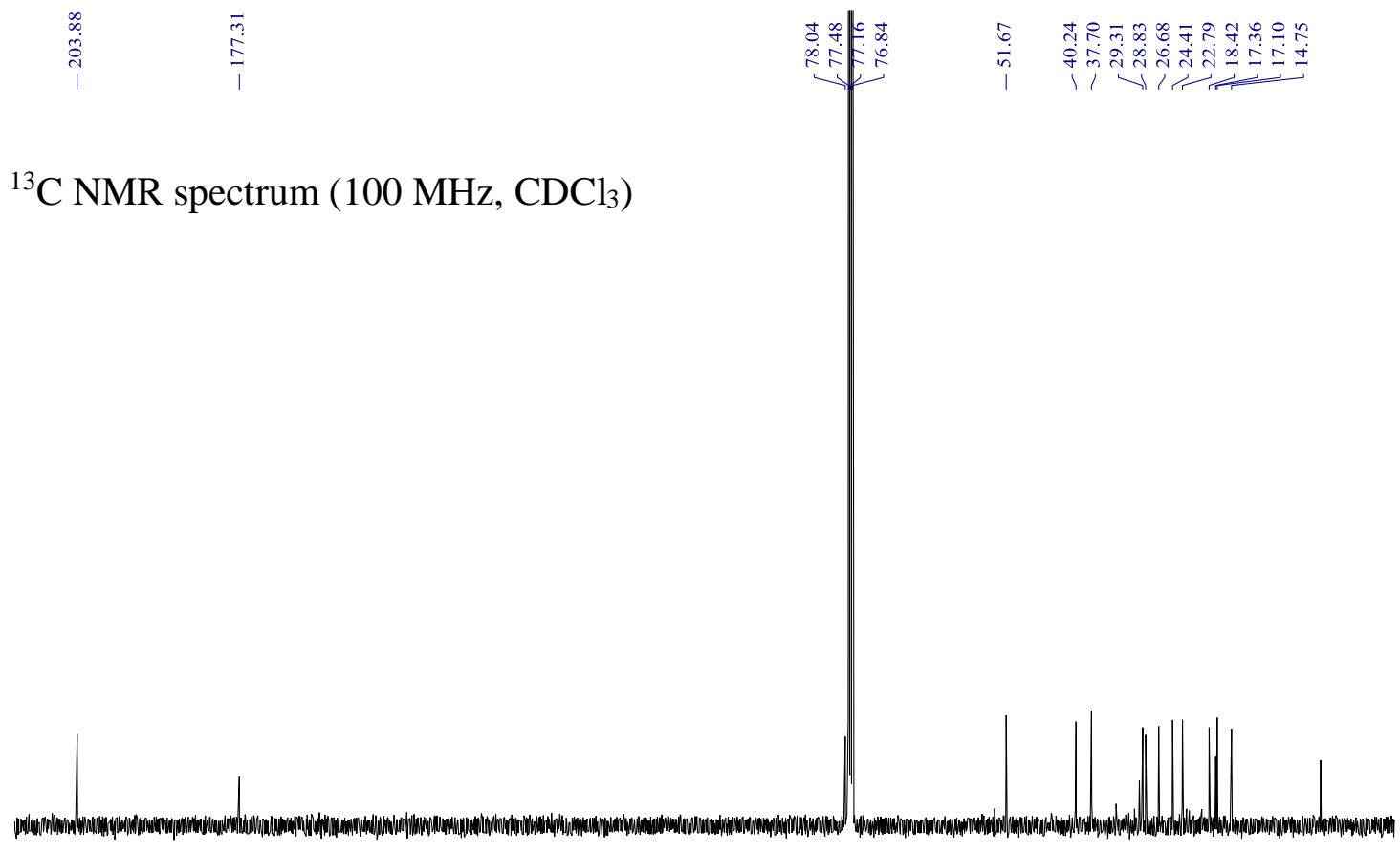

$\begin{array}{lllllllllllllllllllllll}210 & 200 & 190 & 180 & 170 & 160 & 150 & 140 & 130 & 120 & 110 & \begin{array}{c}100 \\ \mathrm{f} 1(\mathrm{ppm})\end{array} & 90 & 80 & 70 & 60 & 50 & 40 & 30 & 20 & 10 & 0 & -1(\end{array}$ 

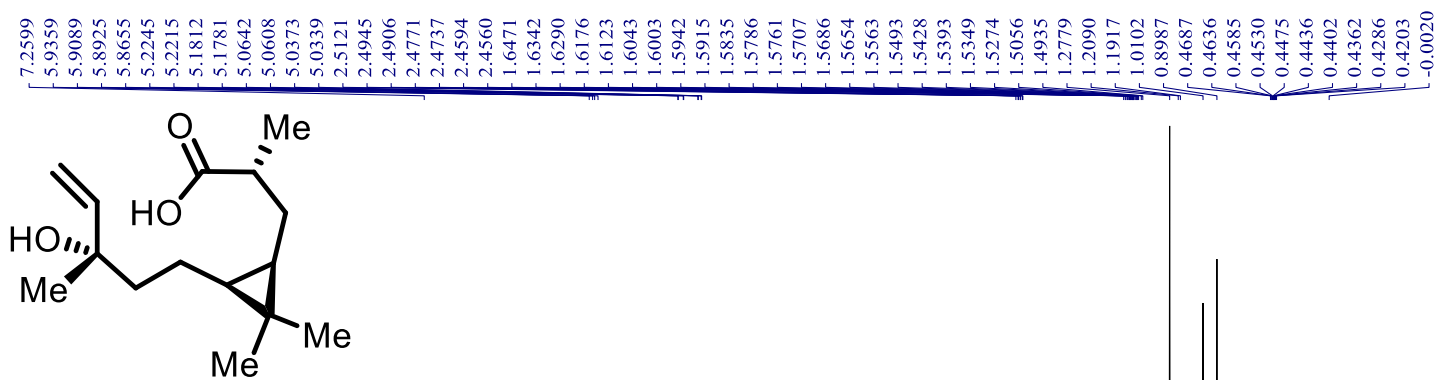

${ }^{1} \mathrm{H}$ NMR spectrum $\left(400 \mathrm{MHz}, \mathrm{CDCl}_{3}\right)$
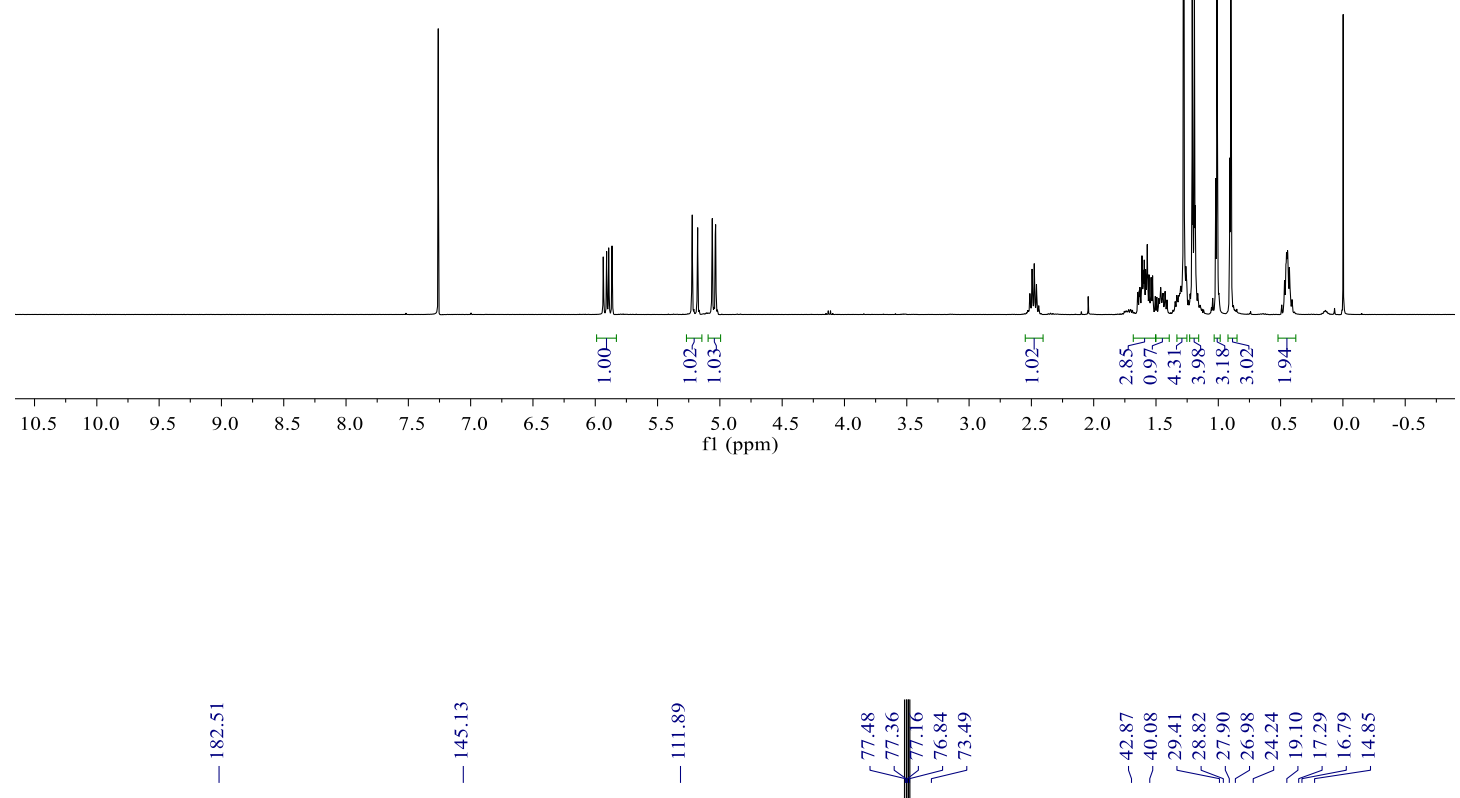

${ }^{13} \mathrm{C}$ NMR spectrum $\left(100 \mathrm{MHz}, \mathrm{CDCl}_{3}\right)$

$\begin{array}{lllllllllll}210 & 200 & 190 & 180 & 170 & 160 & 150 & 140 & 130 & 120 & 110 \\ \mathrm{f} 1(\mathrm{ppm}) & 100\end{array}$ 


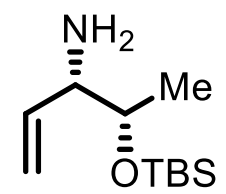

5

${ }^{1} \mathrm{H}$ NMR spectrum $\left(400 \mathrm{MHz}, \mathrm{CDCl}_{3}\right)$

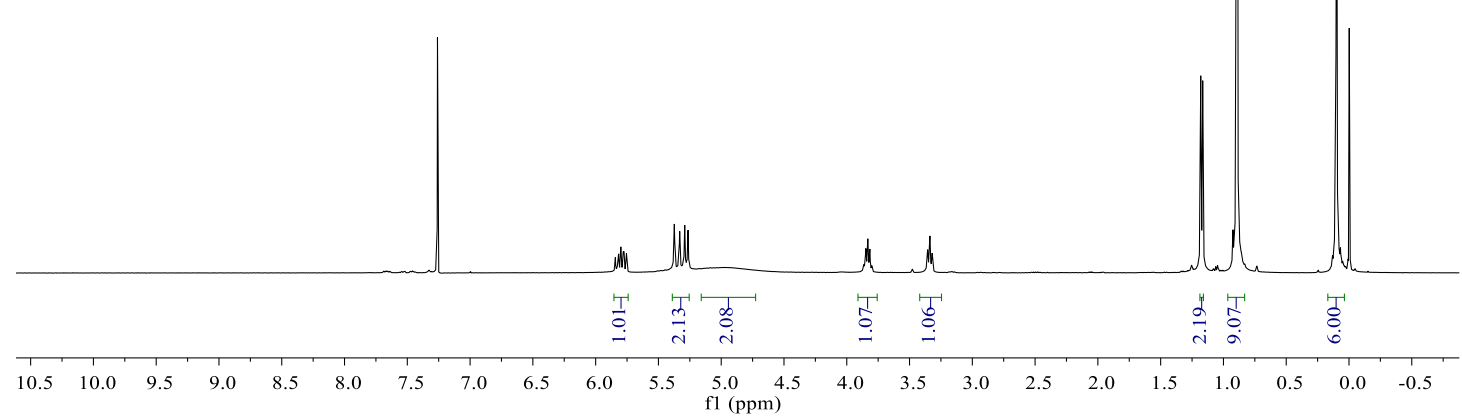

\begin{tabular}{|c|c|c|}
\hline 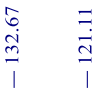 & 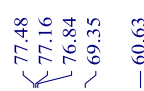 & 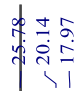 \\
\hline
\end{tabular}

${ }^{13} \mathrm{C}$ NMR spectrum $\left(100 \mathrm{MHz}, \mathrm{CDCl}_{3}\right)$

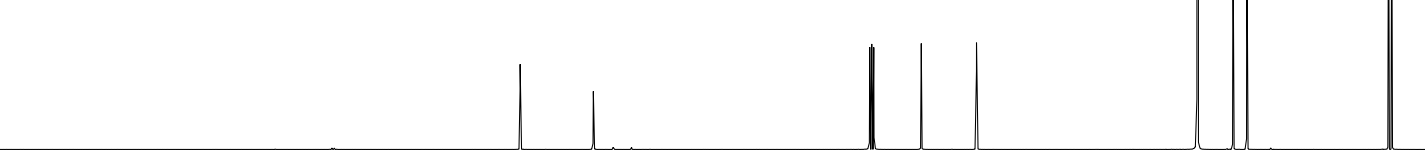

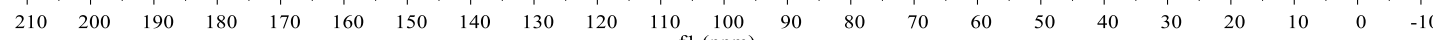



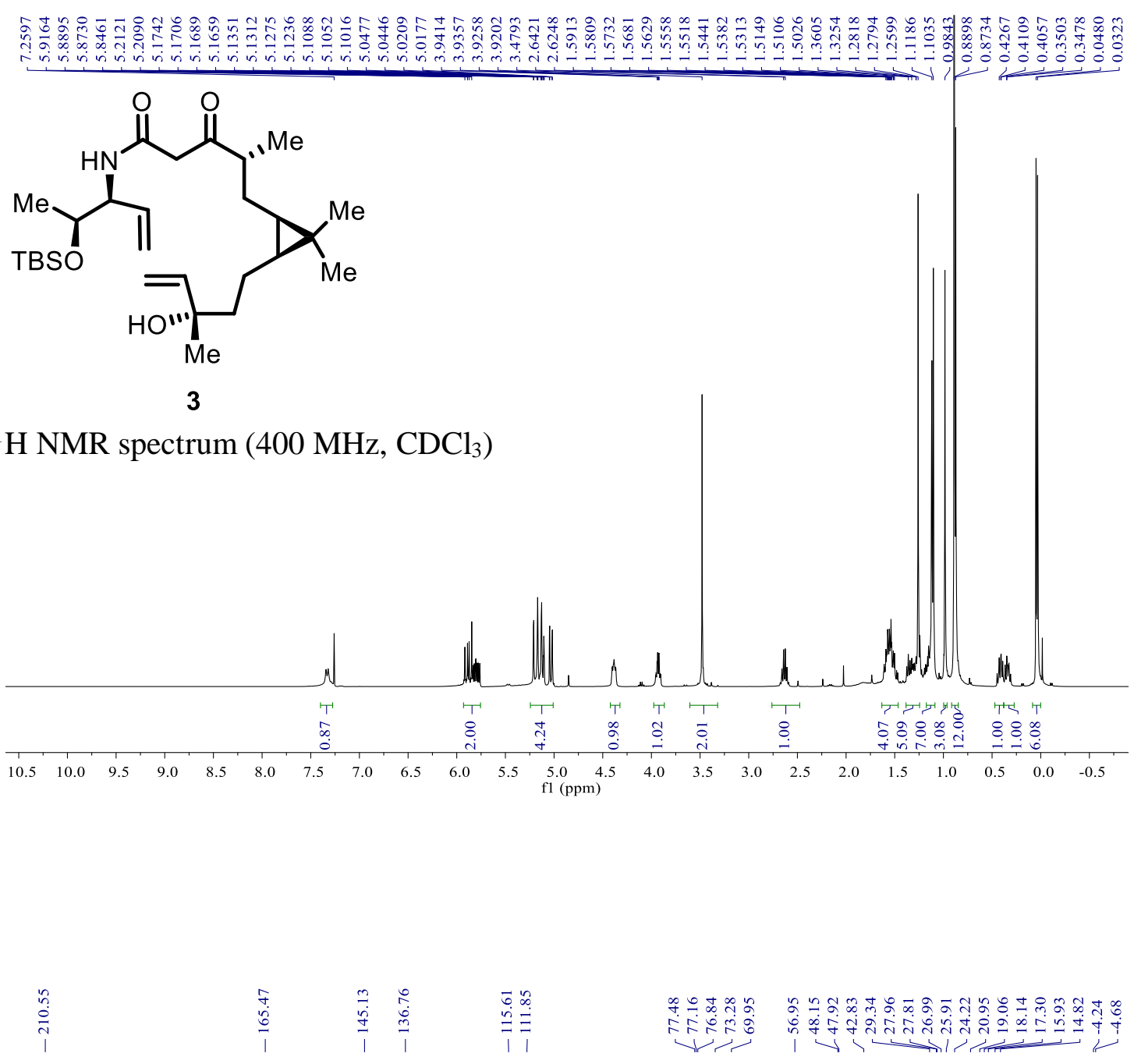

${ }^{13} \mathrm{C}$ NMR spectrum $\left(100 \mathrm{MHz}, \mathrm{CDCl}_{3}\right)$

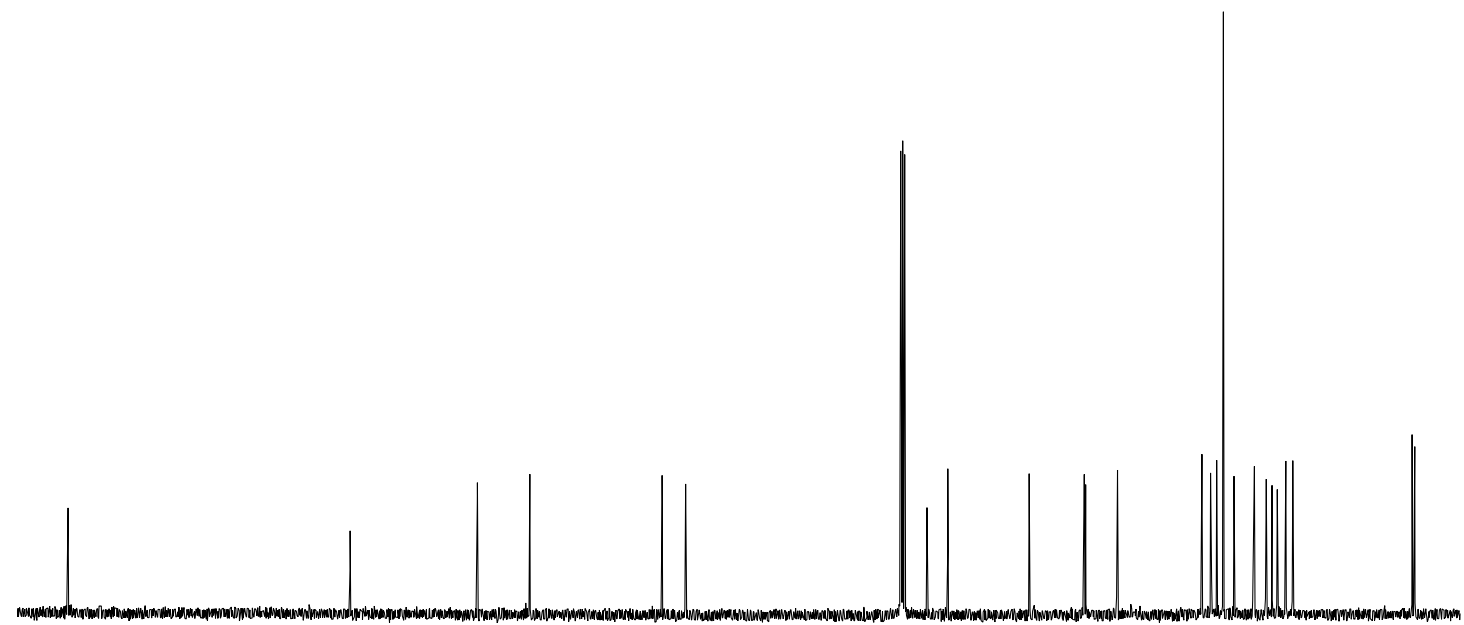

$\begin{array}{llllllllllllllllllllllllll}210 & 200 & 190 & 180 & 170 & 160 & 150 & 140 & 130 & 120 & 110 & 100 & 90 & 80 & 70 & 60 & 50 & 40 & 30 & 20 & 10 & 0 & -11\end{array}$ 


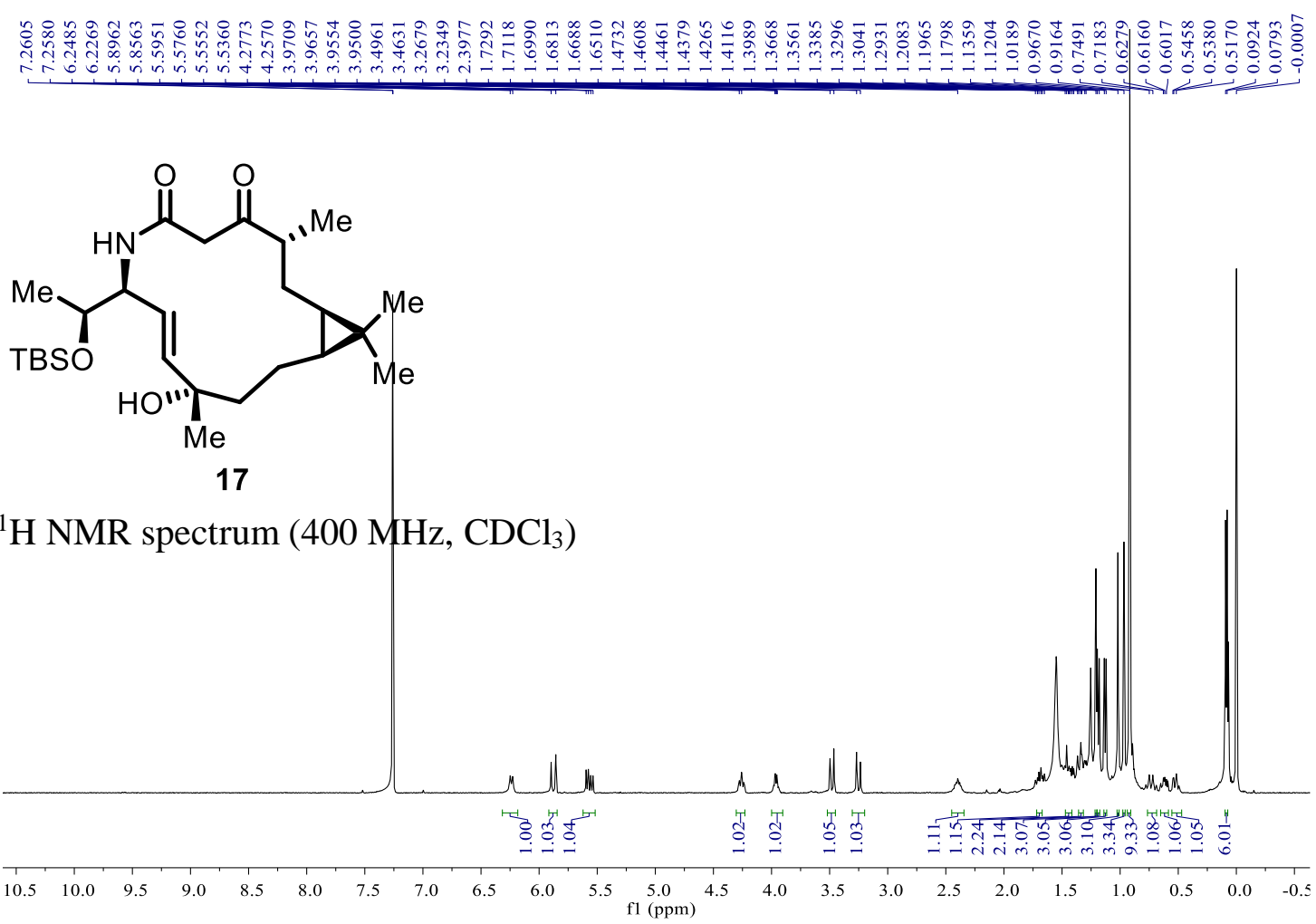

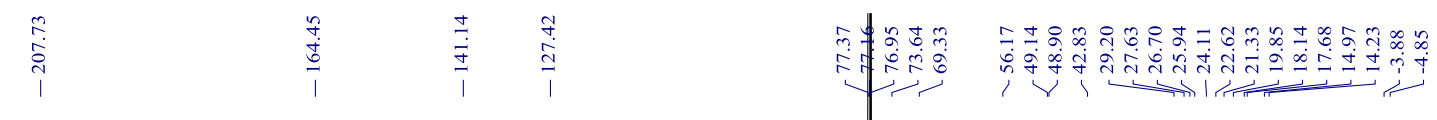

${ }^{13} \mathrm{C}$ NMR spectrum $\left(150 \mathrm{MHz}, \mathrm{CDCl}_{3}\right)$

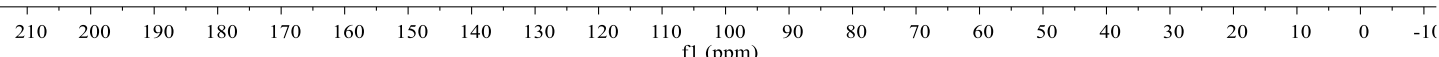


<smiles>C=C[C@H](CS(=O)(=O)c1nnn(-c2ccccc2)n1)NC(=O)O</smiles>

18

${ }^{1} \mathrm{H}$ NMR spectrum $\left(400 \mathrm{MHz}, \mathrm{CDCl}_{3}\right)$

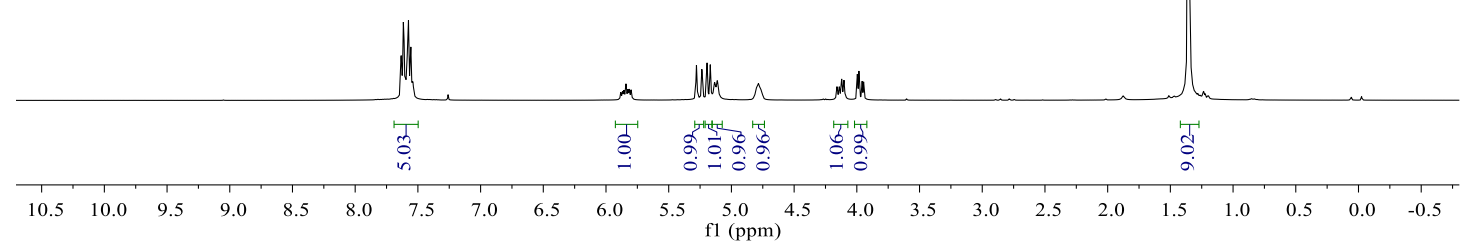

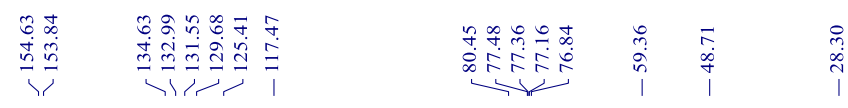

${ }^{13} \mathrm{C}$ NMR spectrum $\left(100 \mathrm{MHz}, \mathrm{CDCl}_{3}\right)$

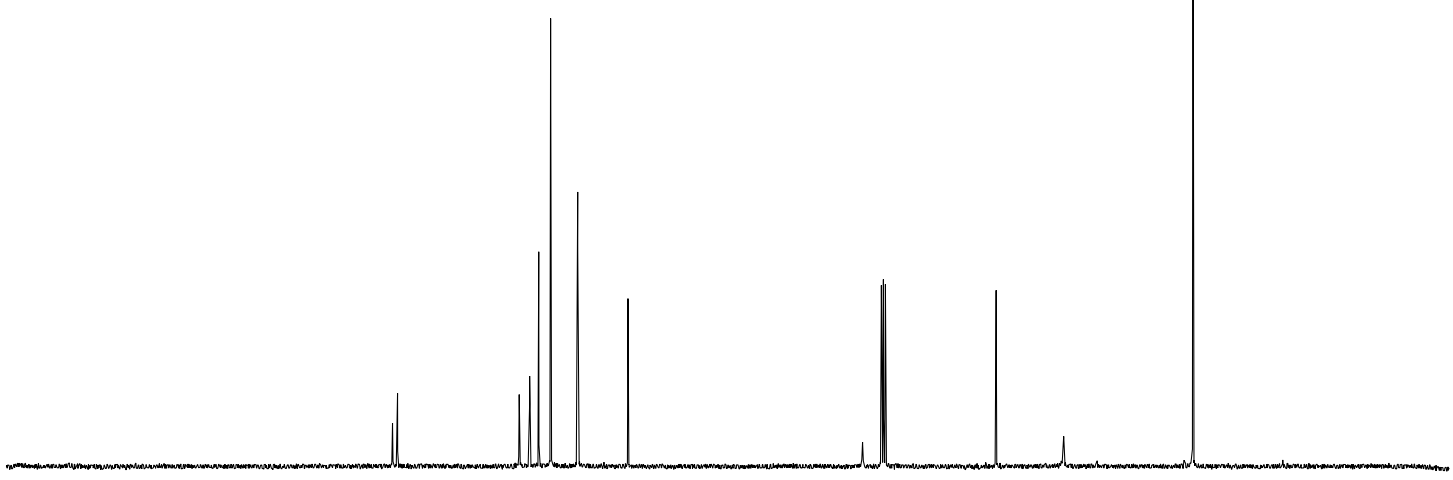

$\begin{array}{llllllllllllllllllllllllllll}210 & 200 & 190 & 180 & 170 & 160 & 150 & 140 & 130 & 120 & 110 & 100 & 90 & 80 & 70 & 60 & 50 & 40 & 30 & 20 & 10 & 0 & -10\end{array}$ 

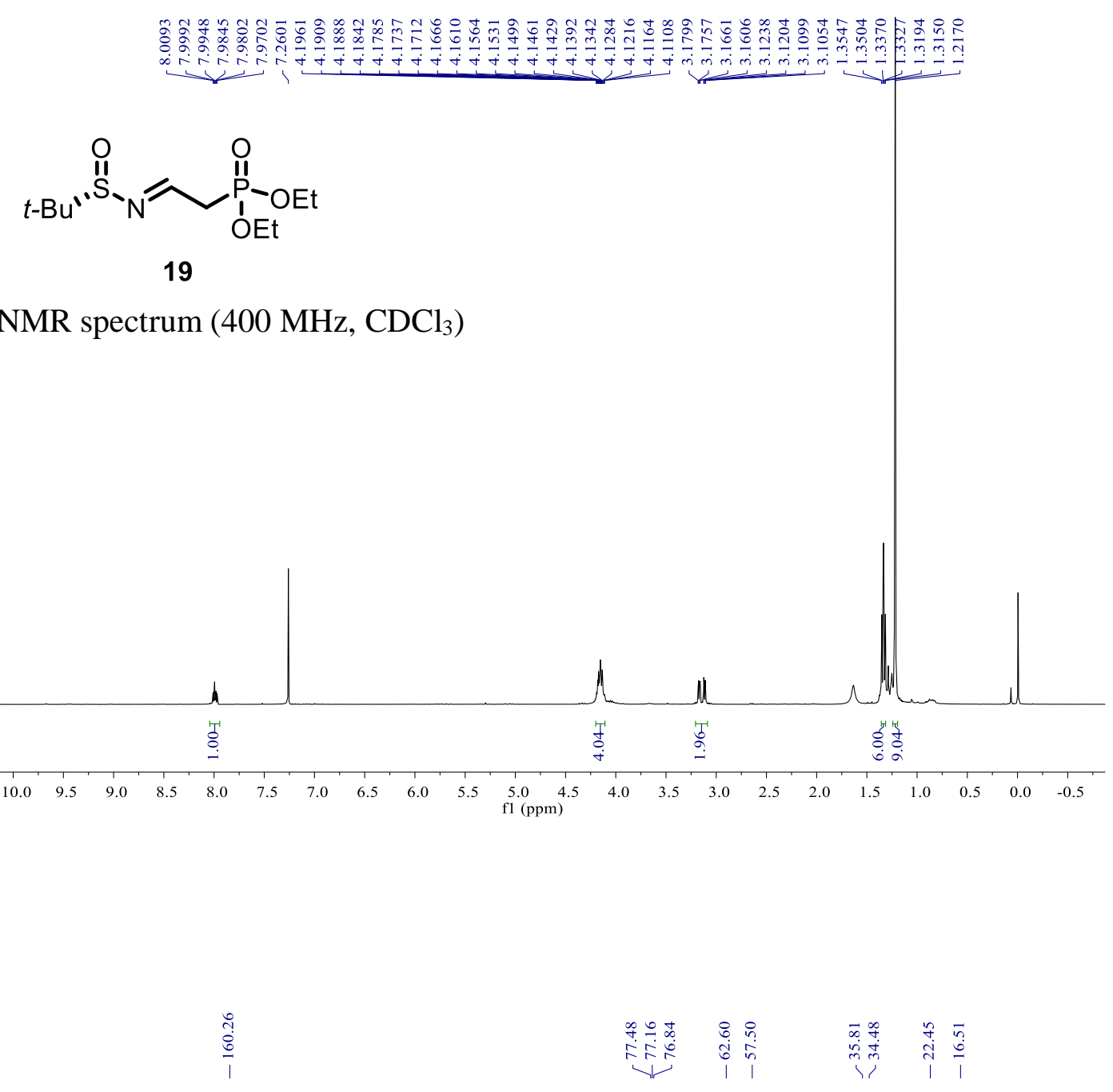

${ }^{13} \mathrm{C}$ NMR spectrum $\left(100 \mathrm{MHz}, \mathrm{CDCl}_{3}\right)$

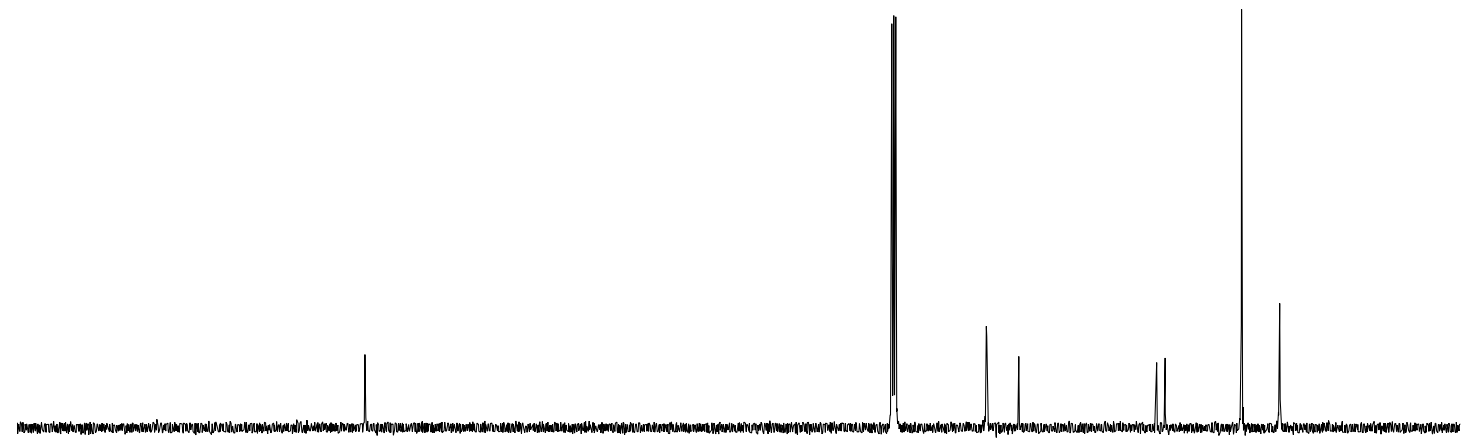

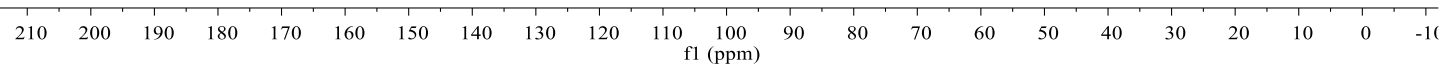




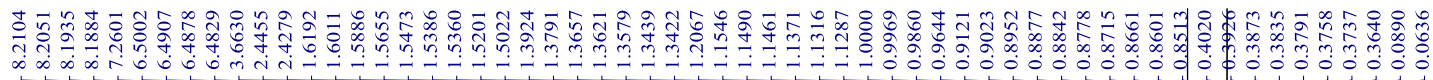

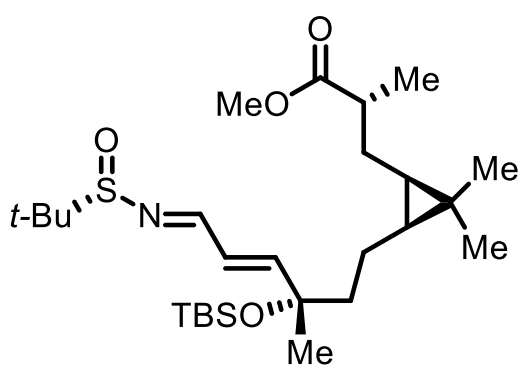

20

${ }^{1} \mathrm{H}$ NMR spectrum $\left(400 \mathrm{MHz}, \mathrm{CDCl}_{3}\right)$
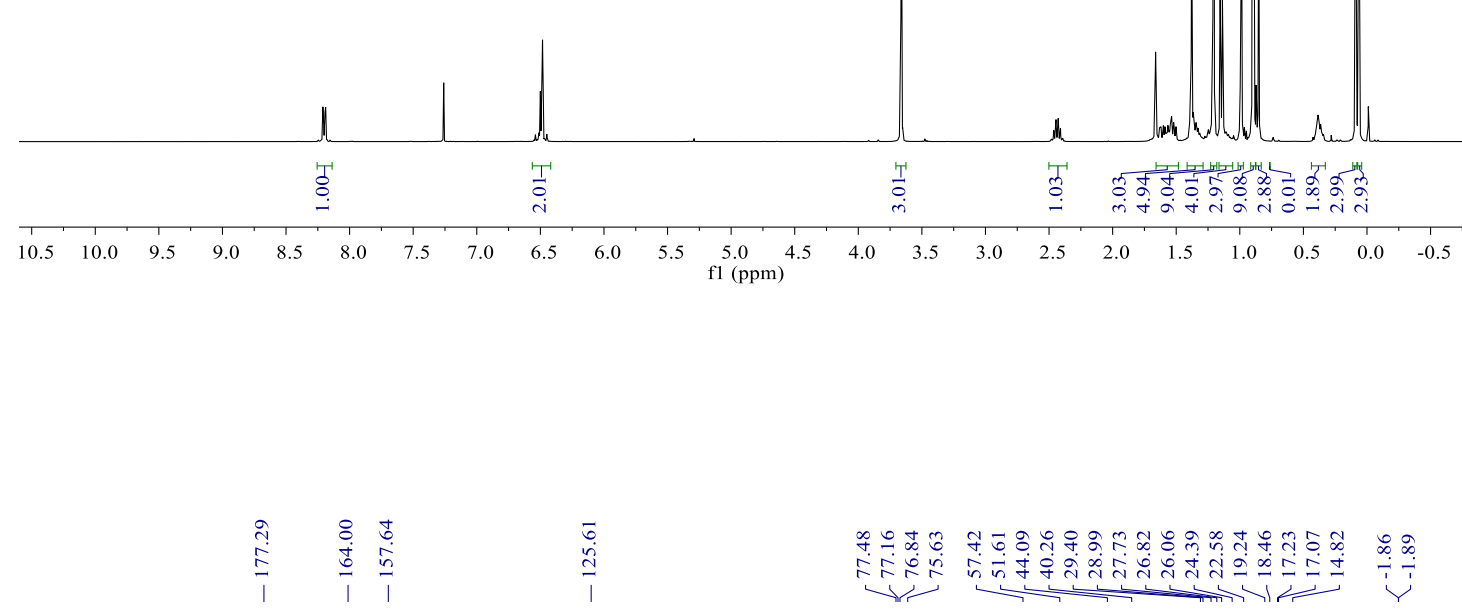

${ }^{13} \mathrm{C}$ NMR spectrum $\left(100 \mathrm{MHz}, \mathrm{CDCl}_{3}\right)$

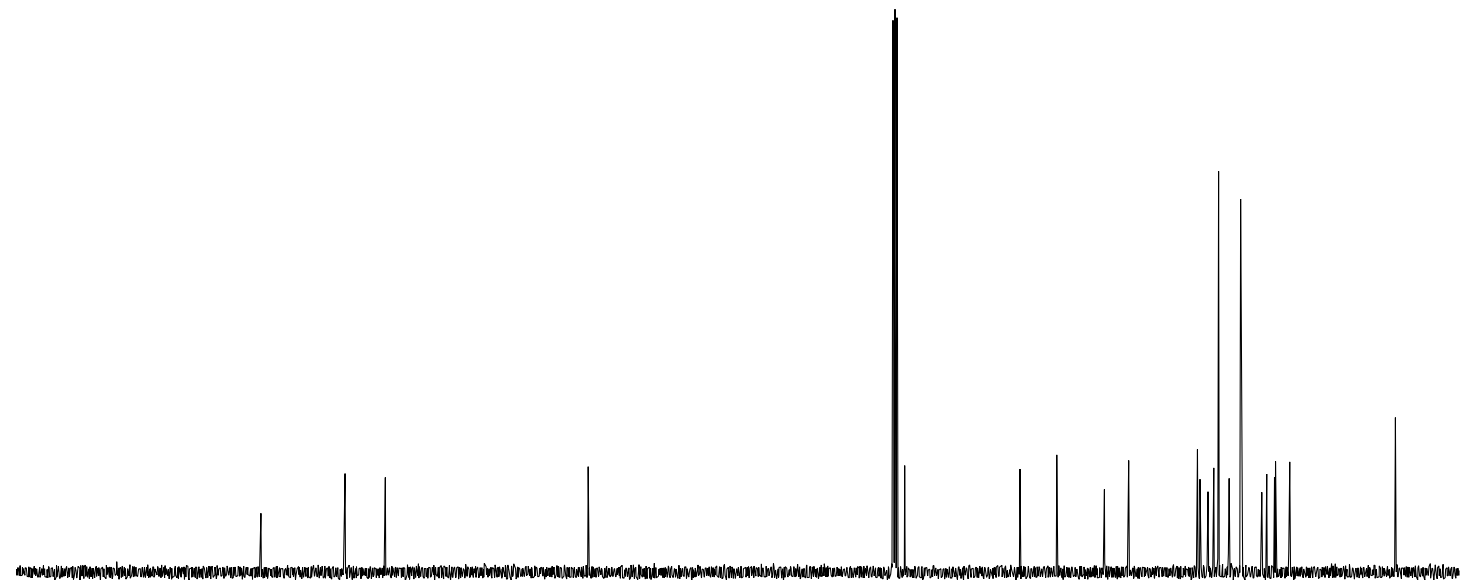

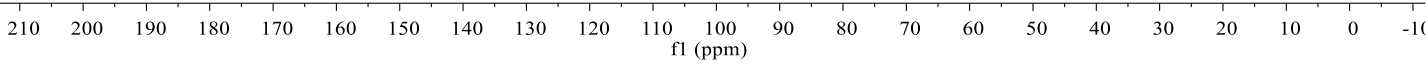



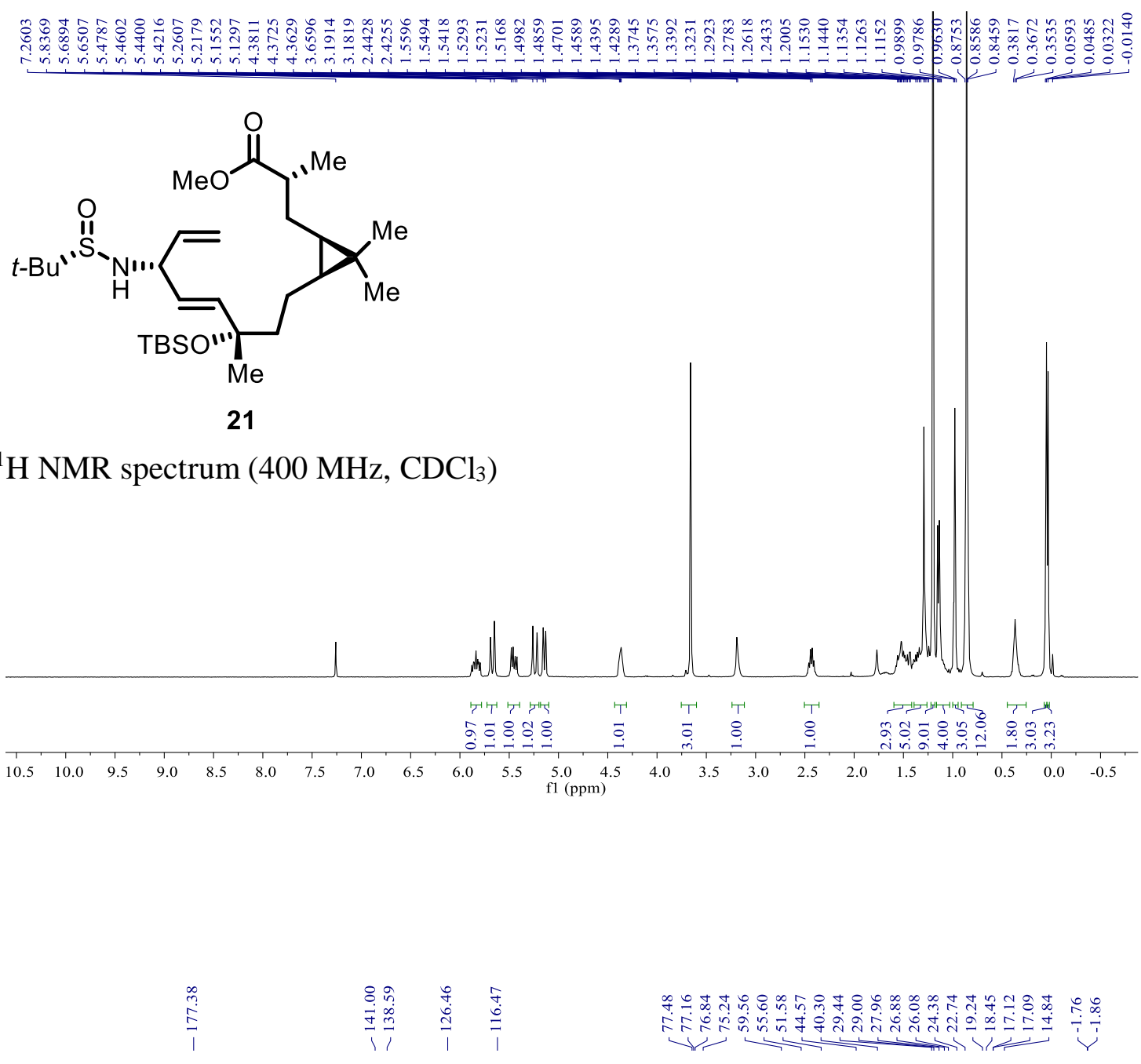

${ }^{13} \mathrm{C}$ NMR spectrum $\left(100 \mathrm{MHz}, \mathrm{CDCl}_{3}\right)$

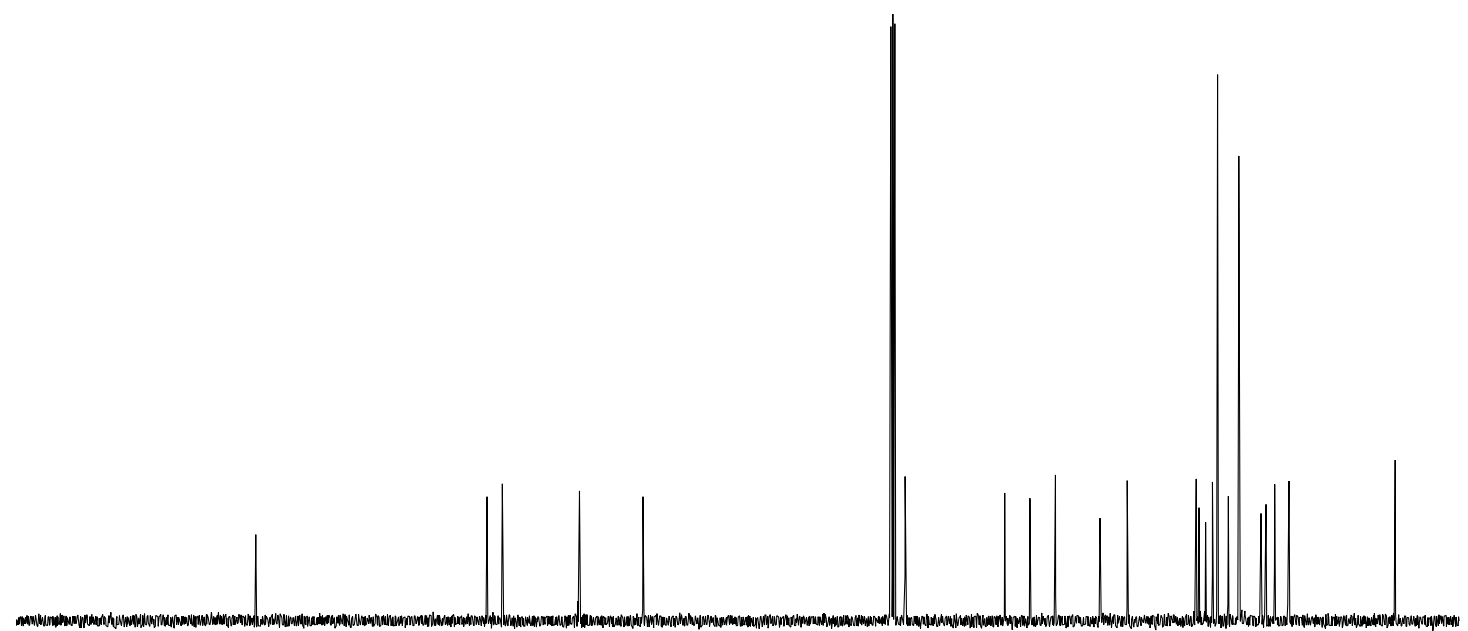

$\begin{array}{llllllllllllllllllllllll}1 & 1 & 200 & 190 & 180 & 170 & 160 & 150 & 140 & 130 & 120 & 110 & 100 & 90 & 80 & 70 & 60 & 50 & 40 & 30 & 20 & 10 & 0 & -1(1\end{array}$ 


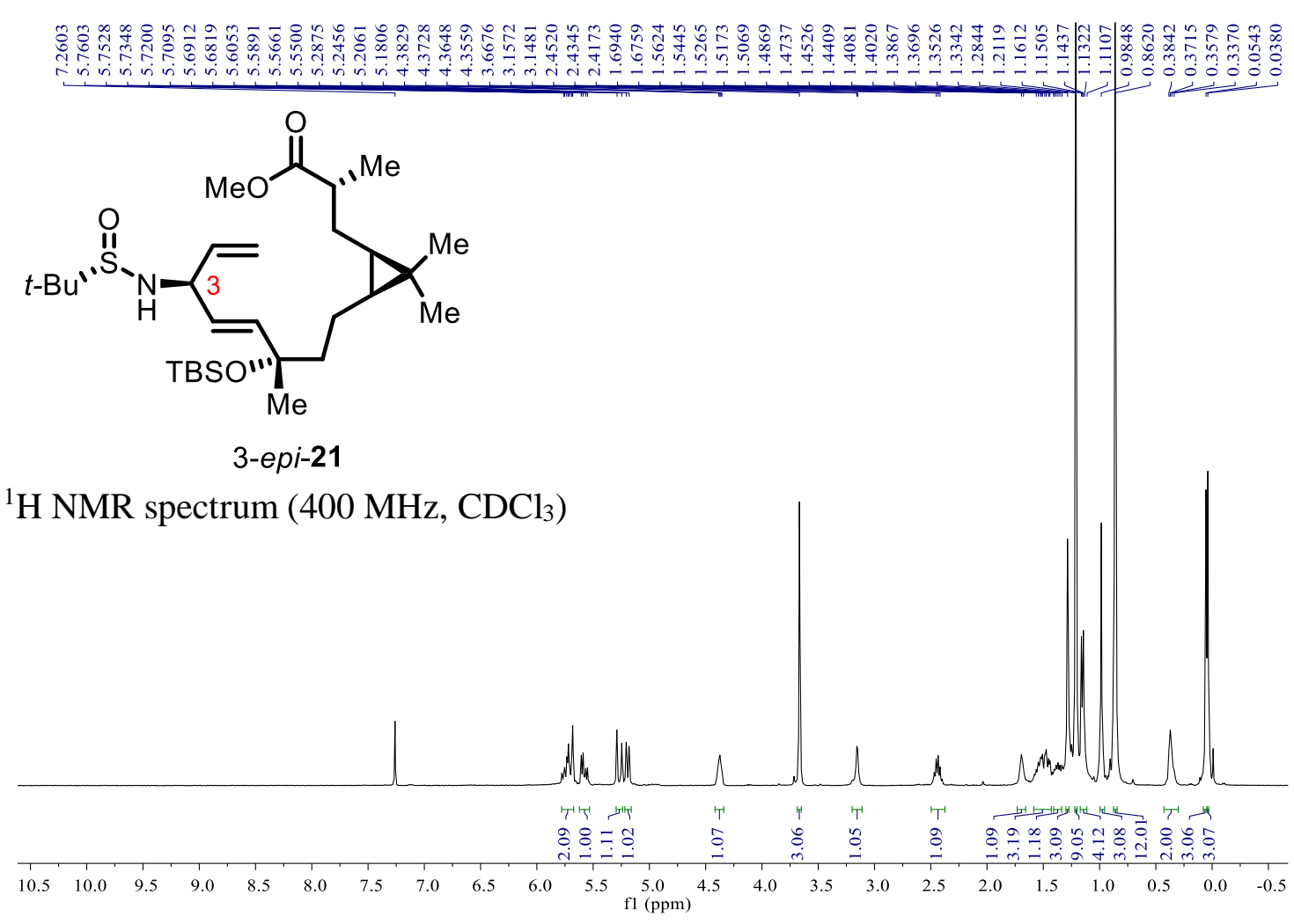

${ }^{13} \mathrm{C}$ NMR spectrum $\left(100 \mathrm{MHz}, \mathrm{CDCl}_{3}\right)$

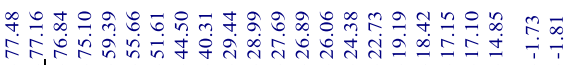

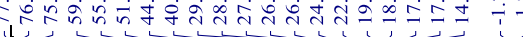

$\begin{array}{lllllllllllllllllllllll}210 & 200 & 190 & 180 & 170 & 160 & 150 & 140 & 130 & 120 & 110 & \begin{array}{l}100 \\ \mathrm{f} 1(\mathrm{ppm})\end{array} & 90 & 80 & 70 & 60 & 50 & 40 & 30 & 20 & 10 & 0 & -1(\mathrm{c})\end{array}$ 


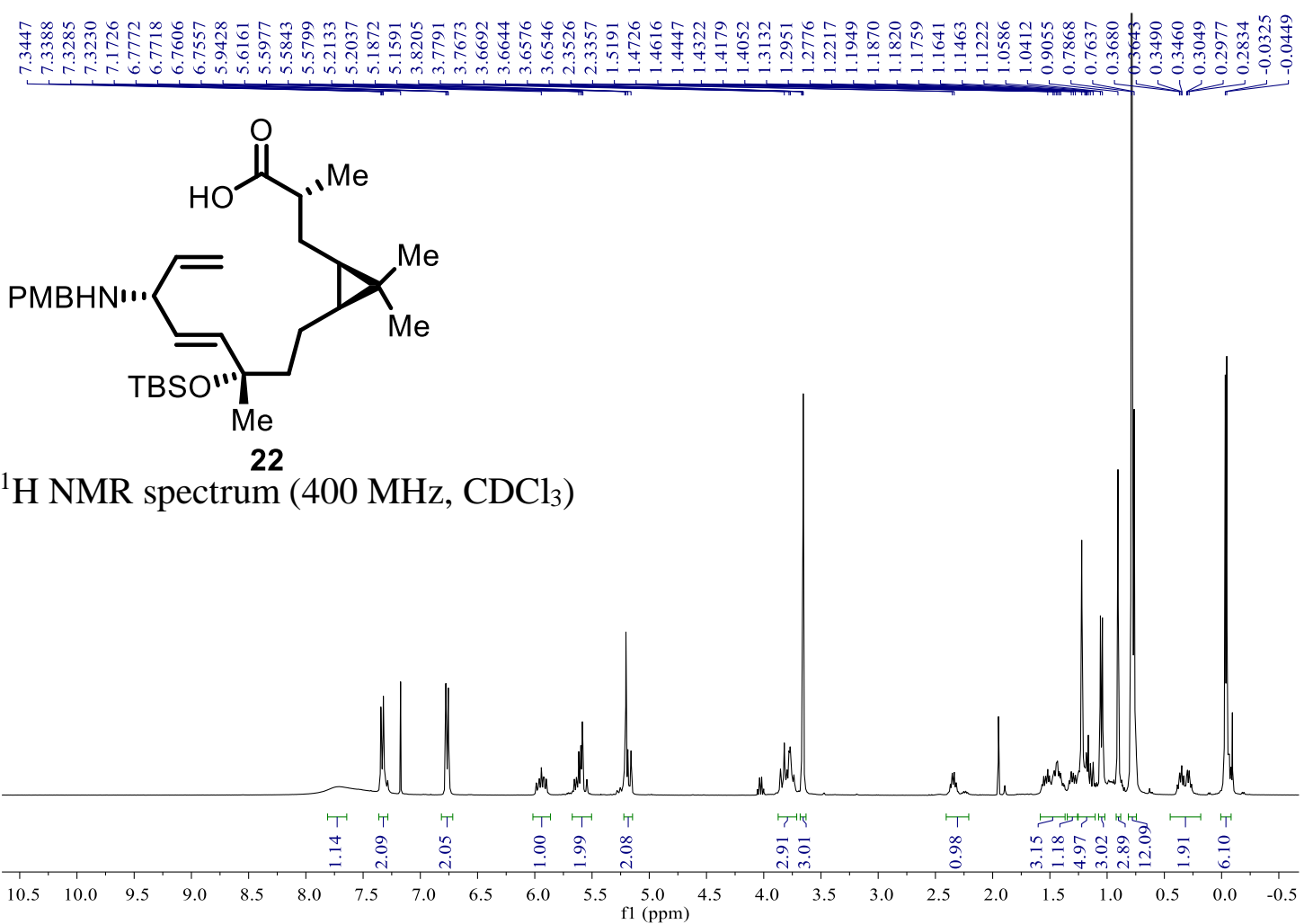

${ }^{13} \mathrm{C} \mathrm{NMR}$ spectrum $\left(100 \mathrm{MHz}, \mathrm{CDCl}_{3}\right)$

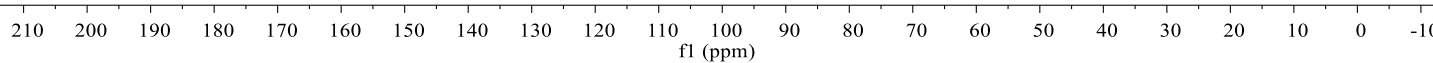




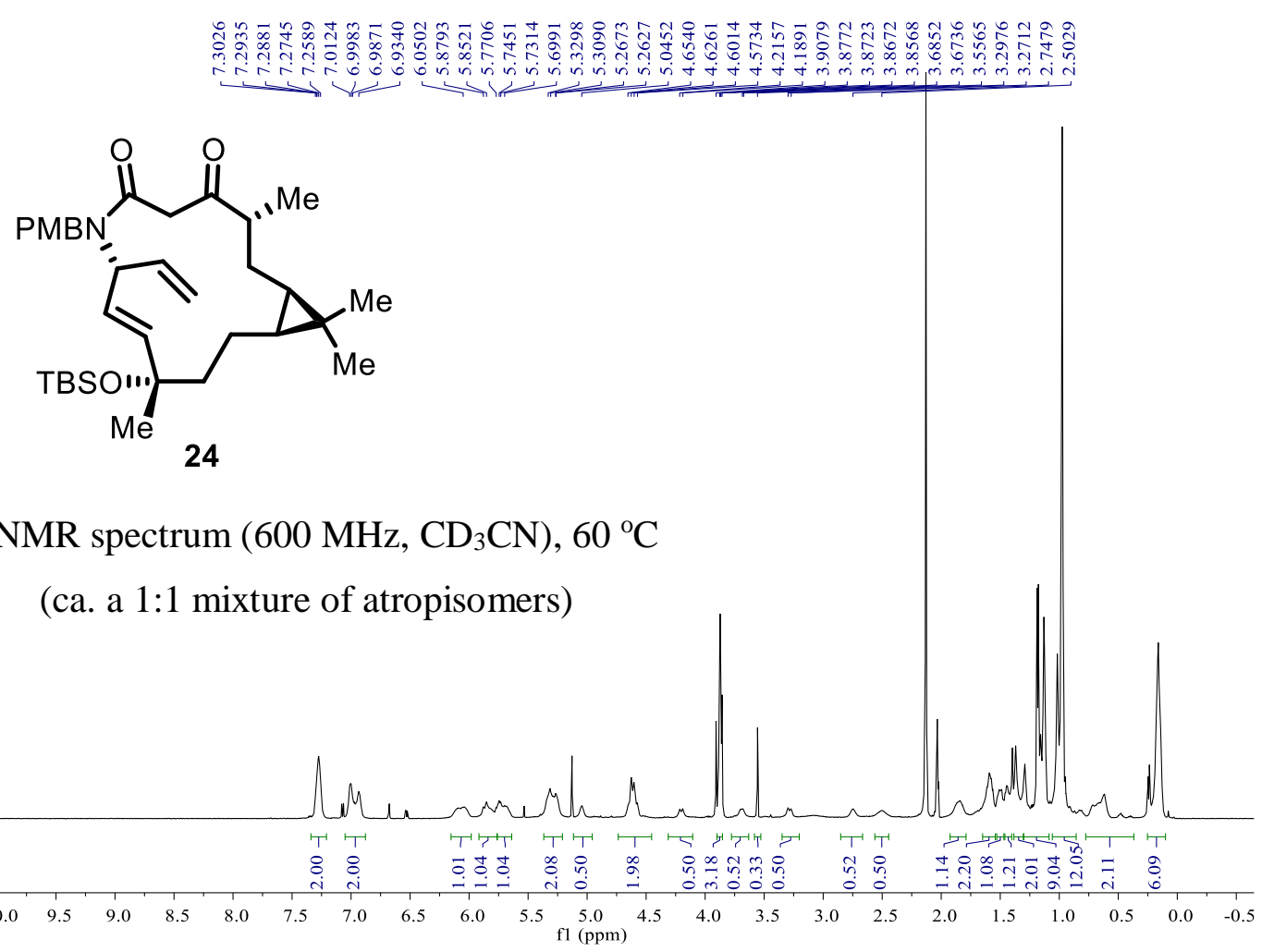

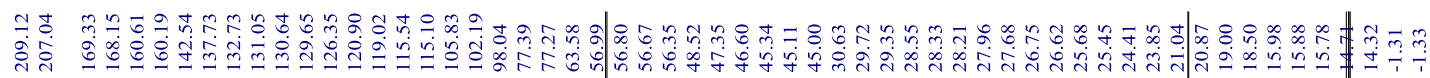

${ }^{13} \mathrm{C}$ NMR spectrum $(150 \mathrm{MHz}, \mathrm{CD} \beta \mathrm{CN}), 60{ }^{\circ} \mathrm{C}$

(ca. a 1:1 mixture of atropisomers)

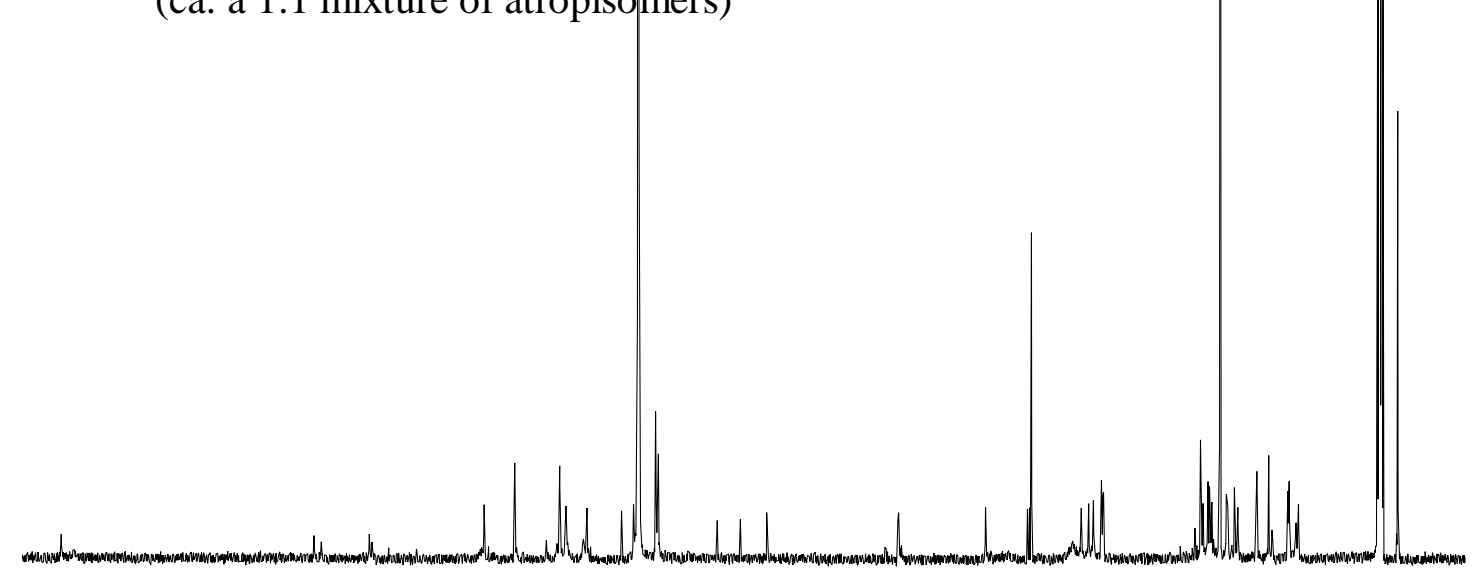

$\begin{array}{llllllllllllllllllllllll}210 & 200 & 190 & 180 & 170 & 160 & 150 & 140 & 130 & 120 & 110 & 100 & 90 & 80 & 70 & 60 & 50 & 40 & 30 & 20 & 10 & 0 & -1(\end{array}$ 


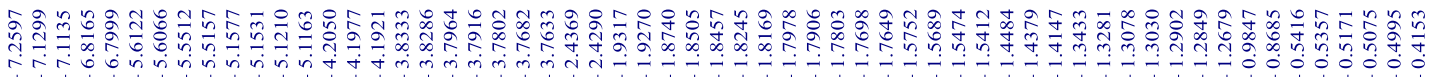

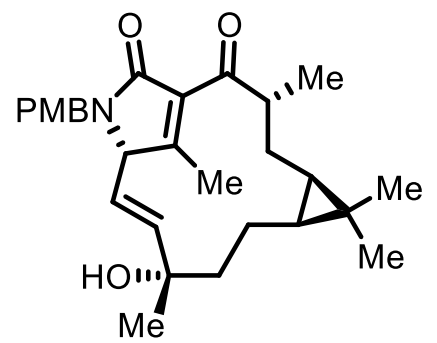

25

${ }^{1} \mathrm{H}$ NMR spectrum $\left(400 \mathrm{MHz}, \mathrm{CDCl}_{3}\right)$

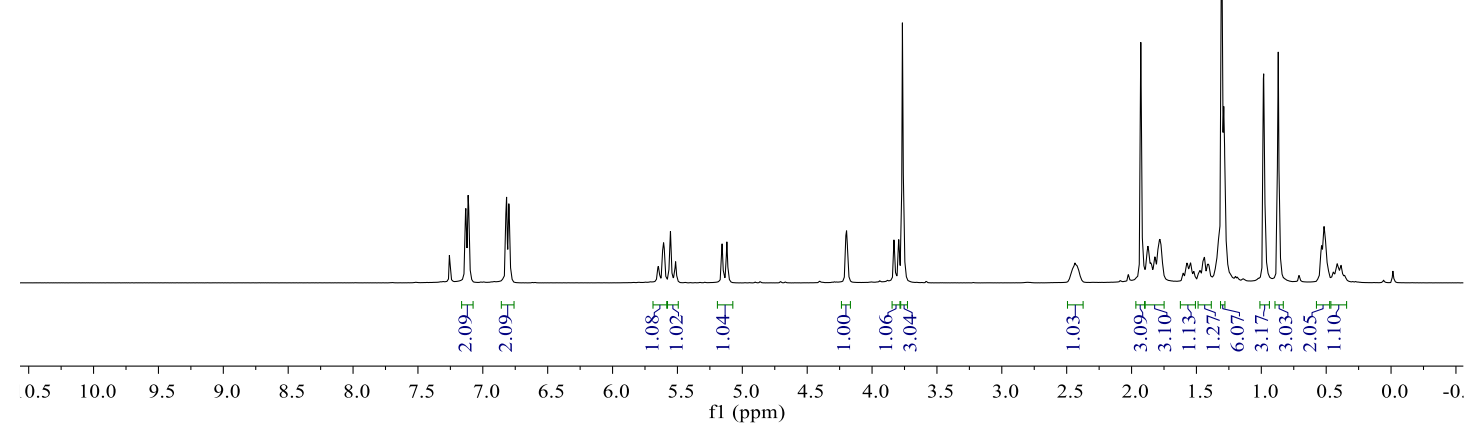

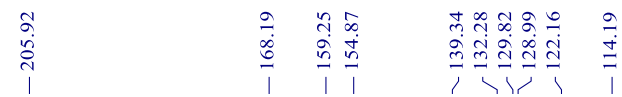

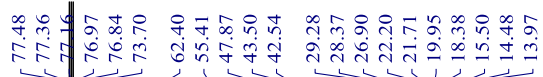

${ }^{13} \mathrm{C}$ NMR spectrum $\left(100 \mathrm{MHz}, \mathrm{CDCl}_{3}\right)$

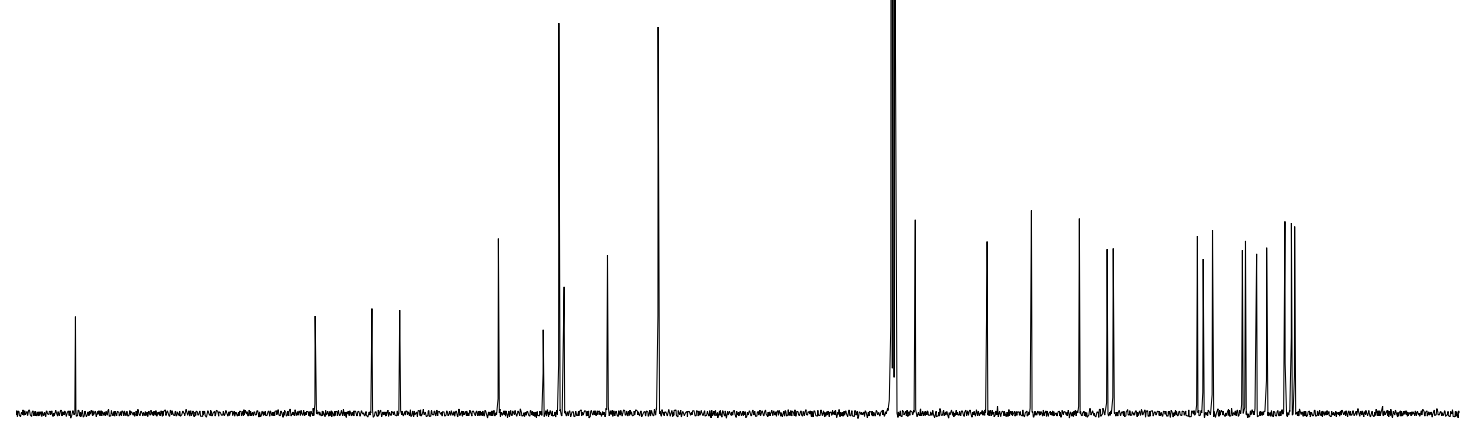

$\begin{array}{lllllllllllllllllllllll}210 & 200 & 190 & 180 & 170 & 160 & 150 & 140 & 130 & 120 & 110 & 100 & 90 & 80 & 70 & 60 & 50 & 40 & 30 & 20 & 10 & 0 & -1(\end{array}$ 


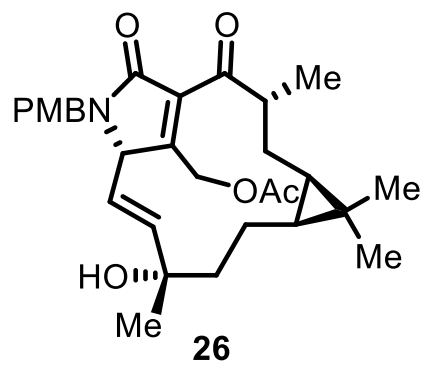

${ }^{1} \mathrm{H}$ NMR spectrum $\left(400 \mathrm{MHz}, \mathrm{CDCl}_{3}\right)$

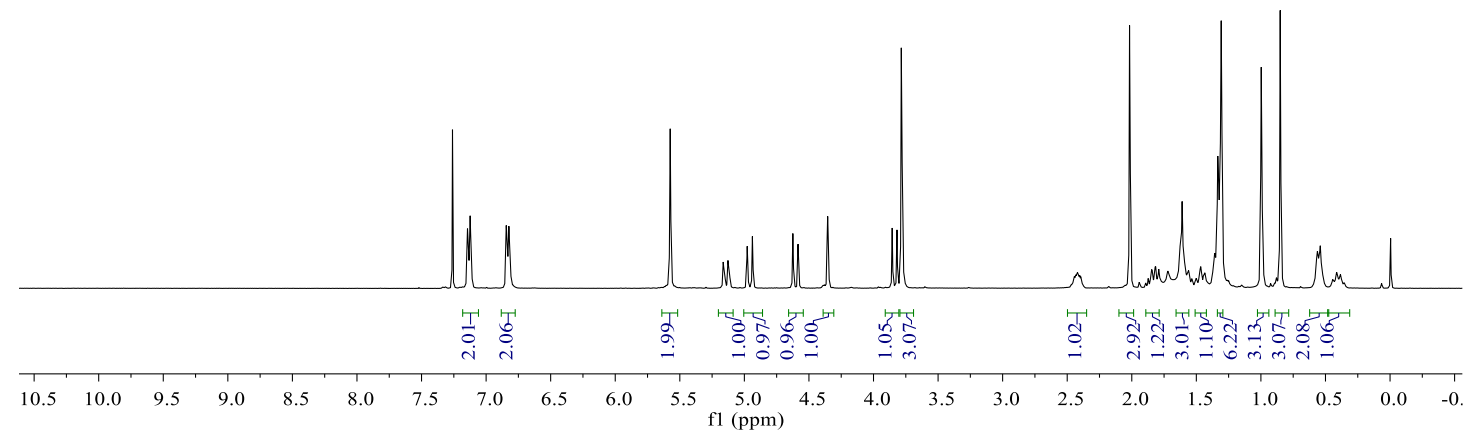

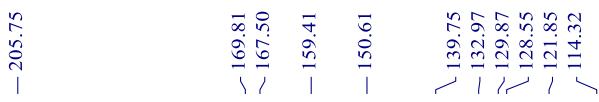

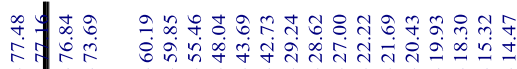

${ }^{13} \mathrm{C}$ NMR spectrum $\left(125 \mathrm{MHz}, \mathrm{CDCl}_{3}\right)$

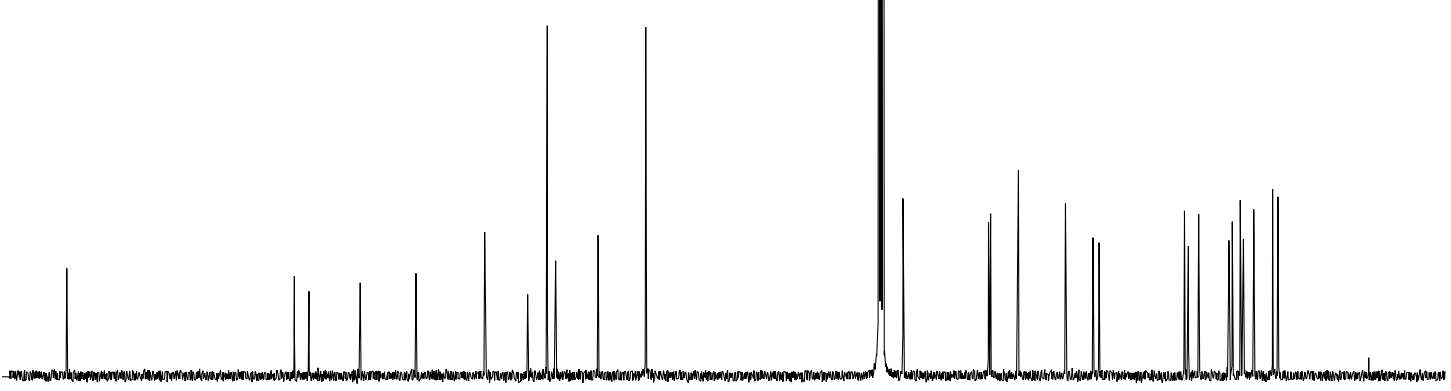

$\begin{array}{llllllllllllllllllllllllll}210 & 200 & 190 & 180 & 170 & 160 & 150 & 140 & 130 & 120 & 110 & 100 & 90 & 80 & 70 & 60 & 50 & 40 & 30 & 20 & 10 & 0 & -1(\end{array}$ 


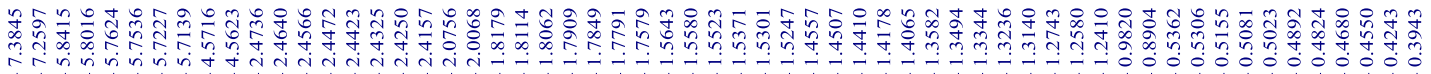

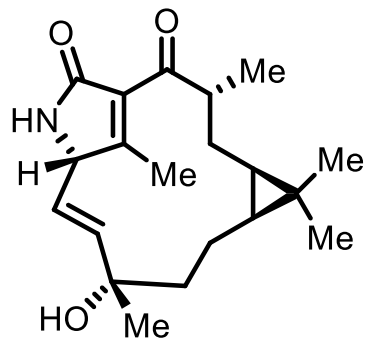

$(+)$-jatrophalactam (1)

${ }^{1} \mathrm{H}$ NMR spectrum (400 MHz, $\mathrm{CDCl}_{3}$ )

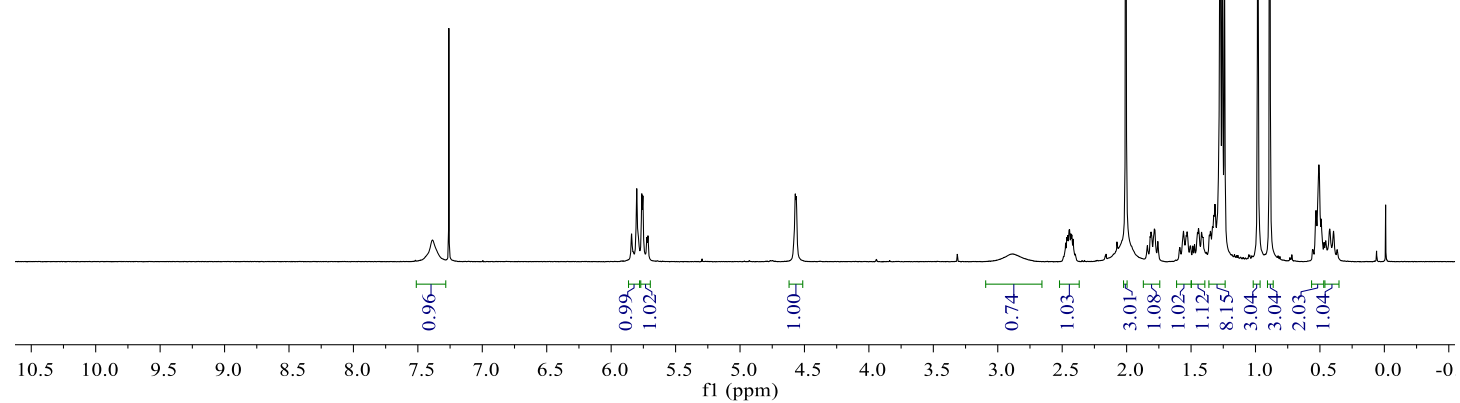

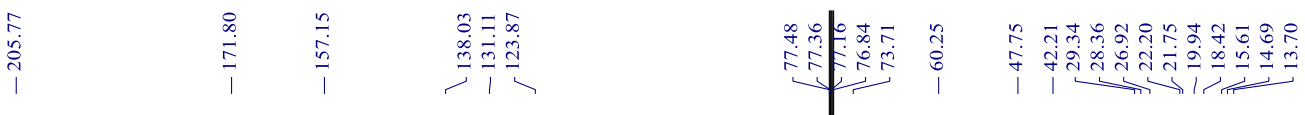

${ }^{13} \mathrm{C}$ NMR spectrum $\left(100 \mathrm{MHz}, \mathrm{CDCl}_{3}\right)$

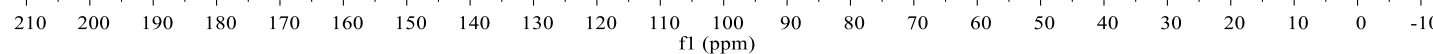



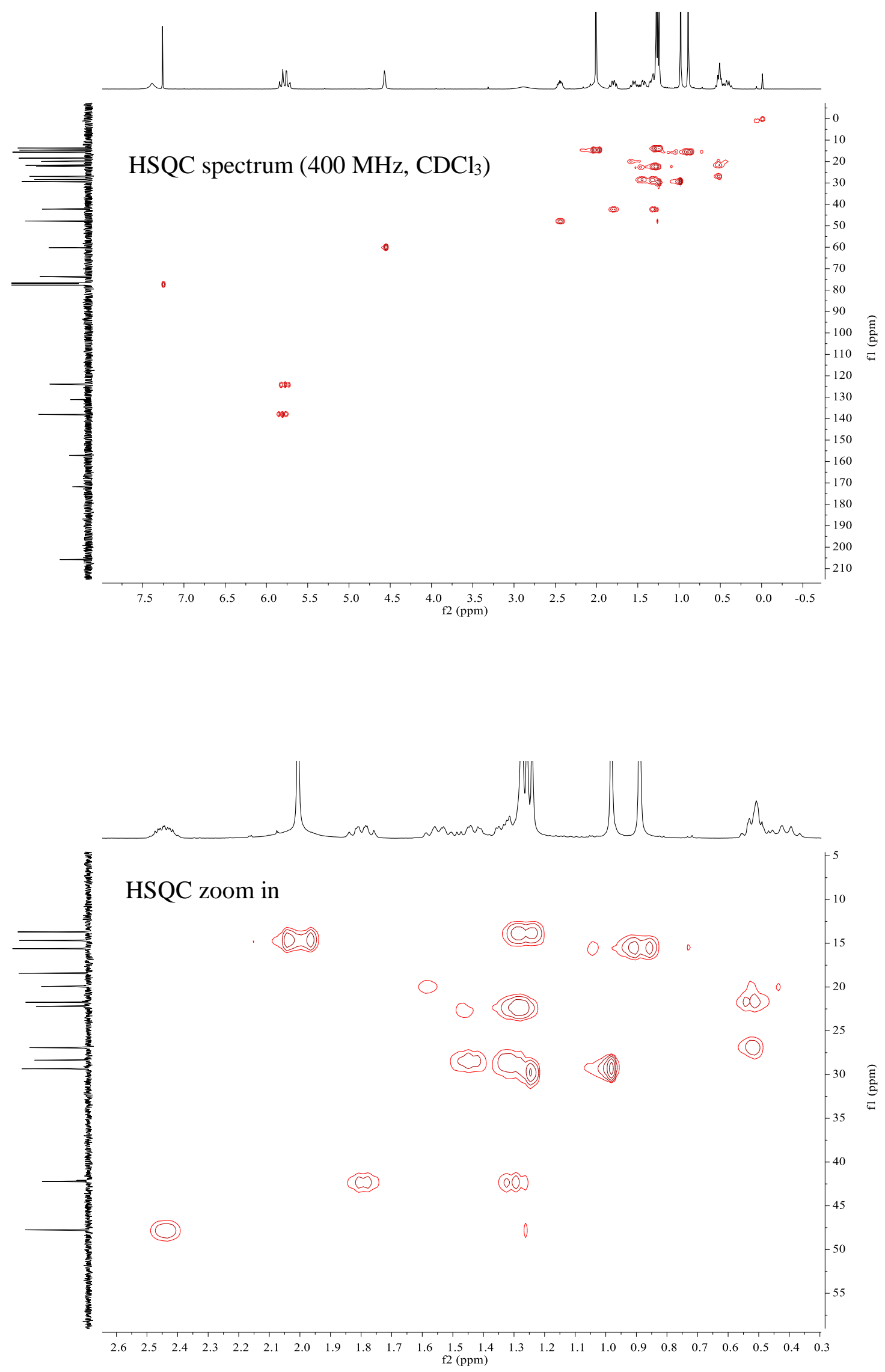
Figure S1. Comparison of ${ }^{1} \mathrm{H}$ NMR Spectra of Synthetic and Natural (+)Jatrophalactam
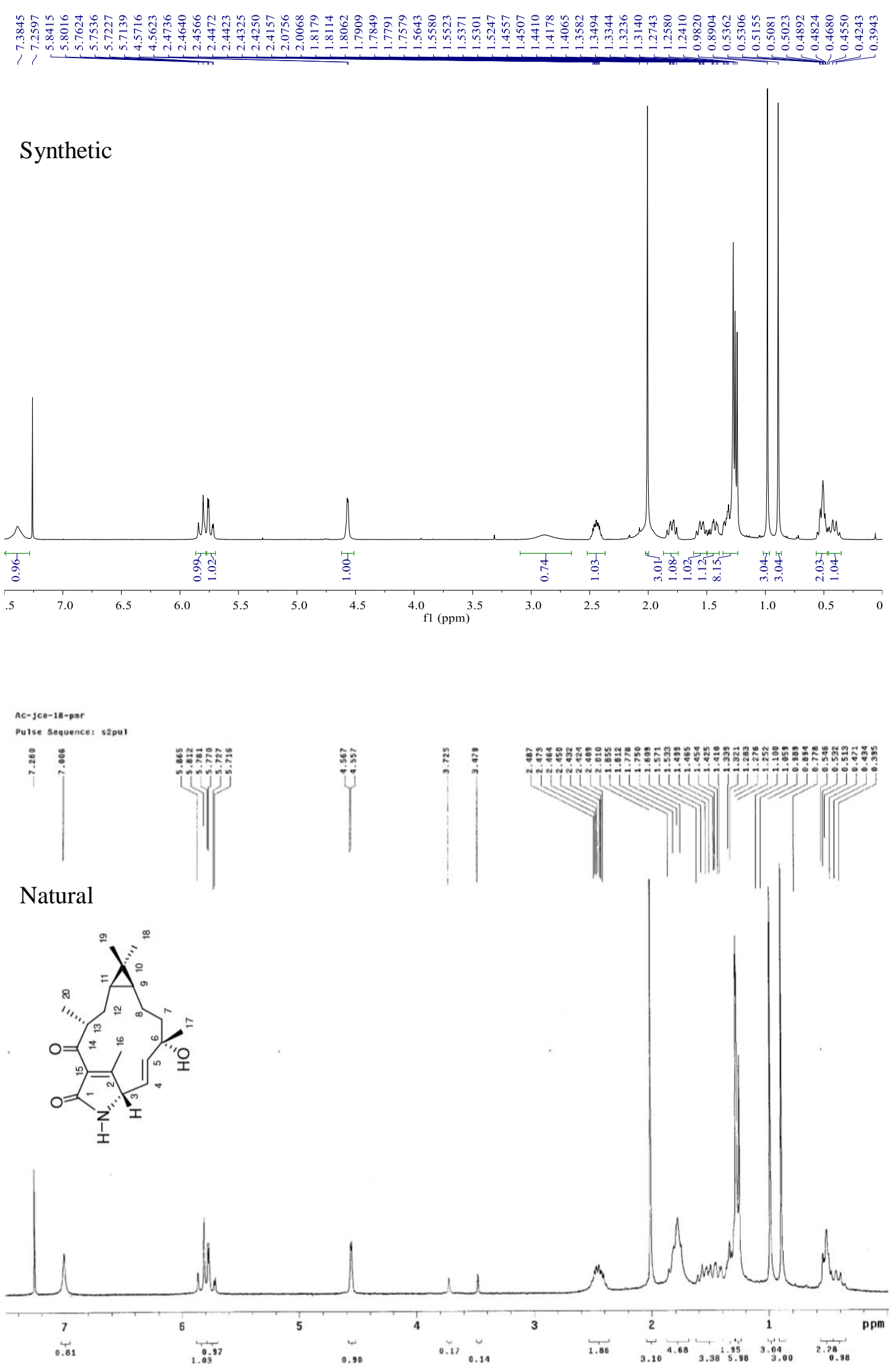
Figure S2. Comparison of ${ }^{13} \mathrm{C}$ NMR Spectra of Synthetic and Natural (+)Jatrophalactam

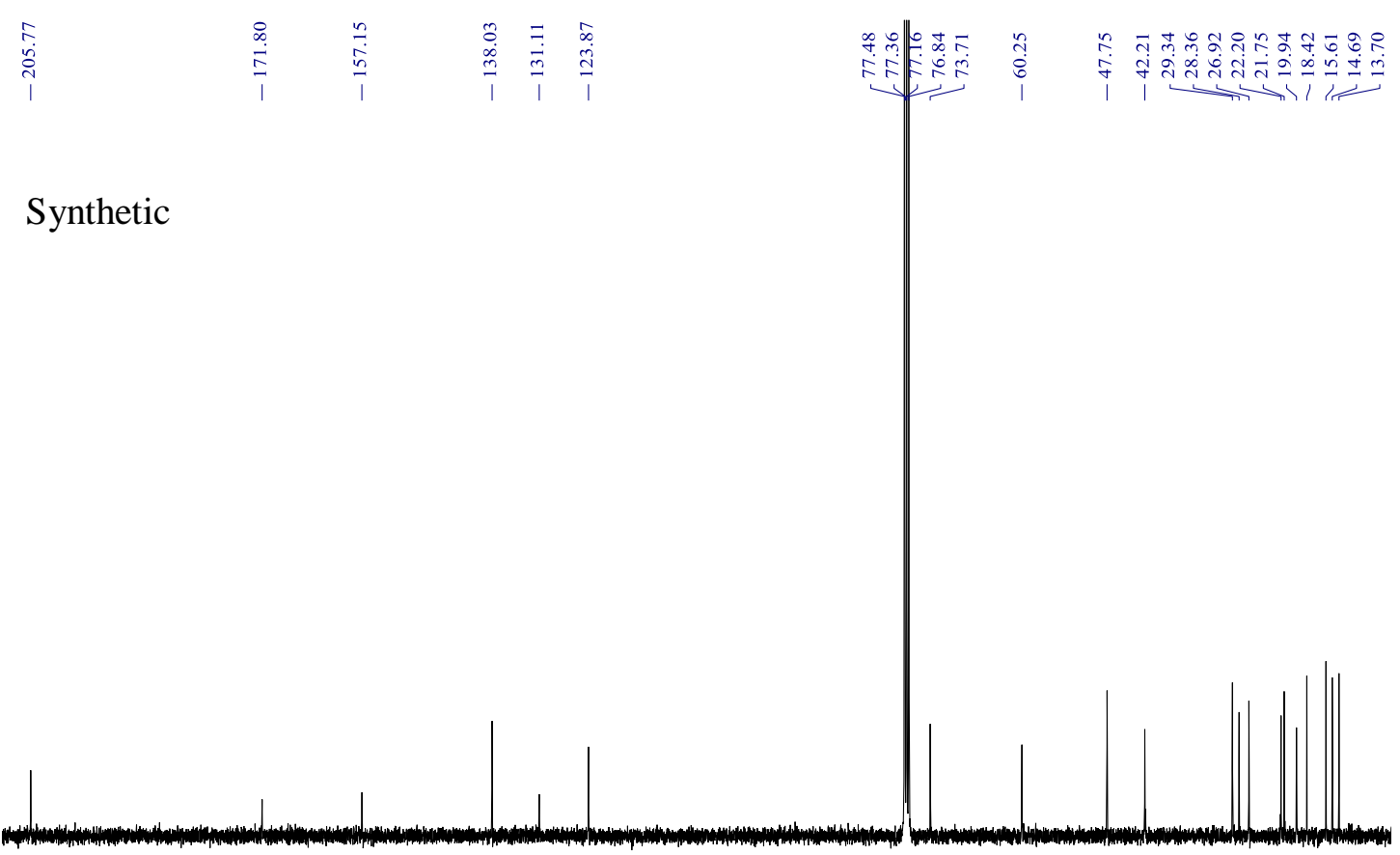

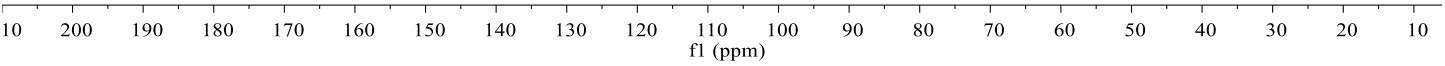

Ac-Jce-18-Car-618

Pulse Sequence: s2pur

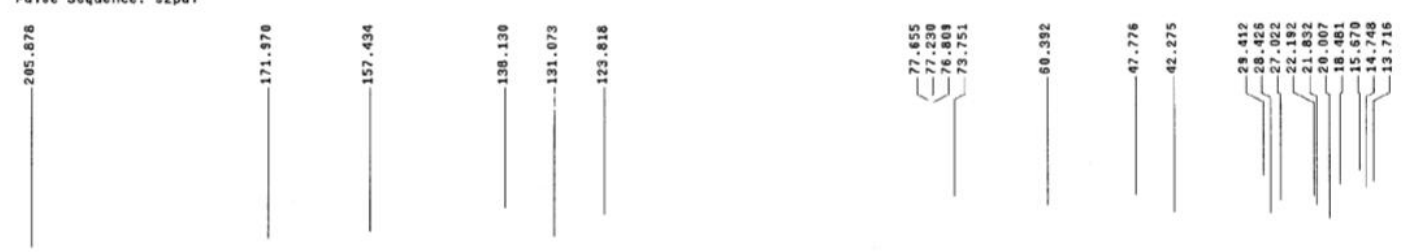

Natural
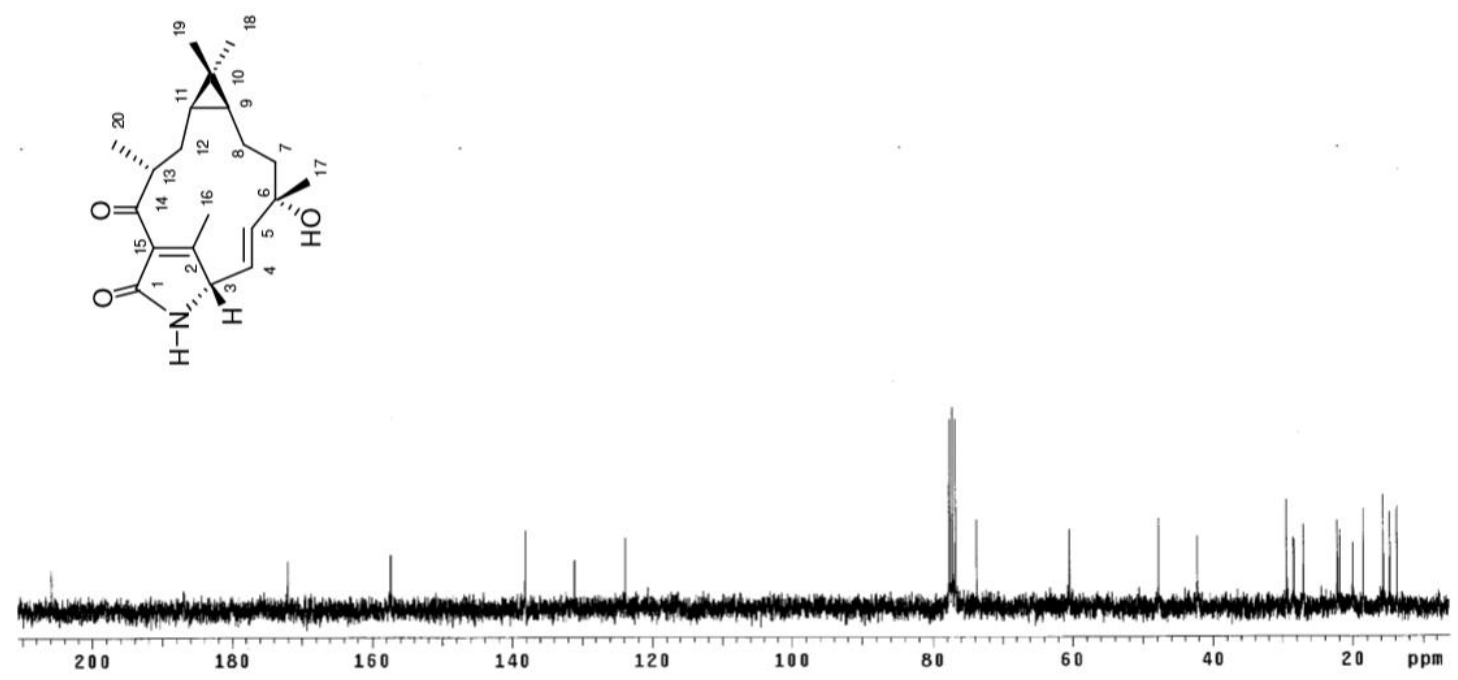